\title{
On the classification of the Samoyedic languages
}

Dedicated to the memory of Eugene Helimski (1950-2007)

The article is introduced by a survey and discussion of previous models of classification of the Samoyedic languages. The main part is devoted to a new model of classification, based on the application of so-called recalibrated glottochronology, including a full documentation of wordlists with etymological remarks, statistics and sources. Finally, the present result is compared with previous models. This article follows the study by Blažek 2012, thus completing the Uralic language family.

For the first time in AD 1096, the ethnonym Samoyed [Самопдb] was used in Tale of Bygone Years (Повпсть врємлнныхъ лютьб), probably about the Nenets (Blažek, Hofírková \& Kovář 2011: 207, 217-218). The first record of words from any Samoyedic language was mediated by a British merchant Richard James. In a manuscript of his Russian Vocabulary from 1618-1620, he included several words identified as Nenets (Stipa 1981: 57-58; Blažek \& Kovář 2013: 279). In his treatise Dissertatio de origine Germanorum from 1710, Gottfried Wilhelm Leibniz proposed that the Samoyedic languages together with Saamic, Finnish, Estonian, Livonian, Komi, and Hungarian had their origin in a language of one great nation of the North (Blažek \& Kovář 2013: 281-282).

Among linguists studying Samoyedic languages there is no consensus concerning their genetic classification. Here we shall compare four models, two of them qualitative and the other two quantitative. In diagram A, the traditional model (Collinder 1960: 11; cf. also Janhunen $1977=\mathrm{J}$ : 7, and Ruhlen 1987: 328, followed by Ethnologue, including even the most recent, 17 th, edition from 2013 - left) is compared with a somewhat revolutionary model by Janhunen (Janhunen 1998: 459 - right), unfortunately presented without any details: 


\section{Václav Blažek}
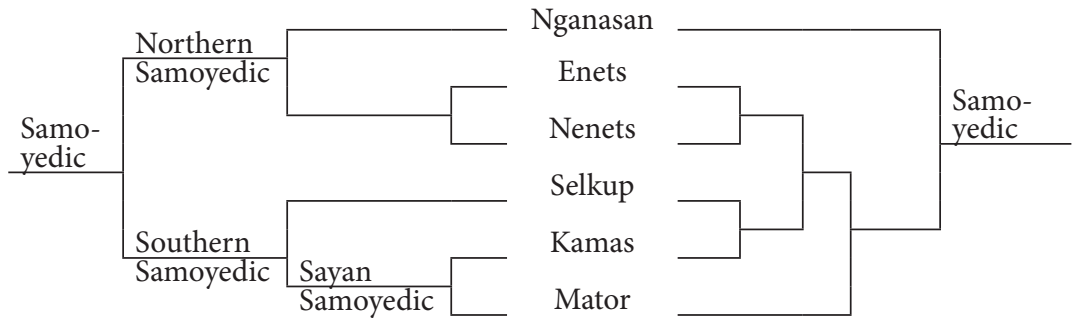

Diagram A: The traditional model (on the left) compared with the model by Janhunen (1998) (on the right)

The alternative models based on application of lexicostatistics and glottochronology were proposed by Helimski (Xelimskij 1982: 39 - left) and Starostin (2004 - right):
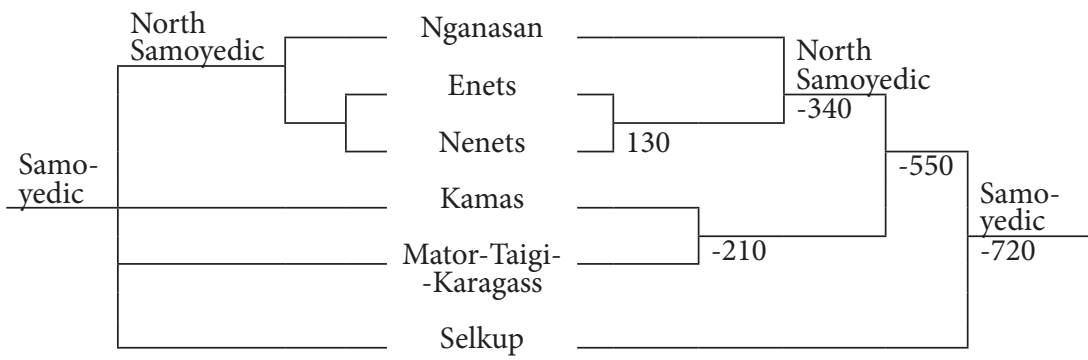

Diagram B: The alternative models by Helimski (left) and Starostin (right)

\begin{tabular}{|l|l|l|l|l|l|}
\hline$\%$ & Enets & Nganasan & Selkup & Kamas & Mator \\
\hline Nenets & $65 / 87=74.7$ & $53 / 86=61.6$ & $49 / 87=56.3$ & $49 / 87=56.3$ & $50 / 86=58.1$ \\
\hline Enets & & $62 / 86=72.1$ & $52 / 87=59.8$ & $51 / 87=58.6$ & $50 / 86=58.1$ \\
\hline Nganasan & & & $47 / 86=54.6$ & $47 / 86=54.6$ & $46 / 85=54.1$ \\
\hline Selkup & & & & $51 / 87=58.6$ & $44 / 86=51.2$ \\
\hline Kamas & & & & & $50 / 86=58.1$ \\
\hline
\end{tabular}

Table 1: Results of Helimski (Xelimskij 1982: 39)

Helimski's model operates with four coordinate branches, although his published results (Xelimskij 1982: 39), applying the standard cladistic procedure based on average values (left side of Diagram C), give another conclusion, namely three coordinate branches: North Samoyedic, KamasMator, Selkup, while the procedure operating with minimum values leads to the binarily organized structure (right side): 
On the classification of the Samoyedic languages

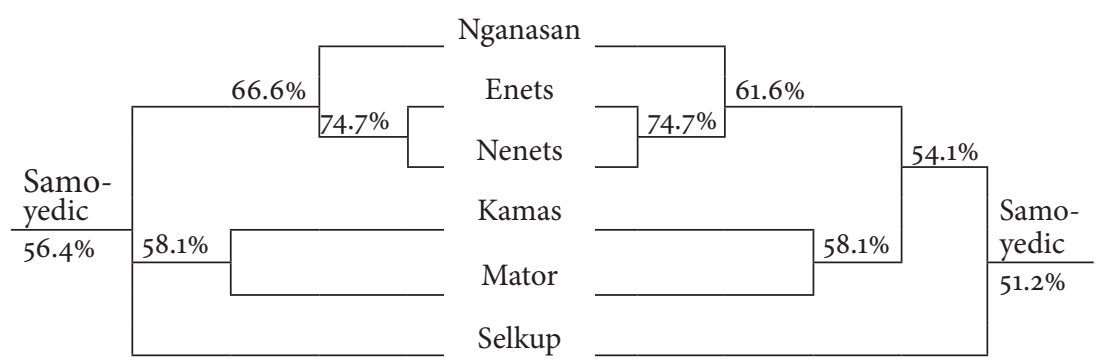

Diagram C: The models based on real results of Helimski, applying the average (left) and minimum (right) values.

The results of the present study, whose methodology is described in Blažek 2012, 81-91, are summarized in Table 2:

\begin{tabular}{|l|l|l|l|l|l|}
\hline$\%$ & Enets & Nganasan & Selkup & Kamas & Mator \\
\hline Nenets & $84 / 98=85.71$ & $71 / 98=72.44$ & $73 / 98=74.49$ & $62.5 / 92=67.93$ & $68 / 91=74.72$ \\
\hline Enets & & $75 / 97=77.32$ & $70 / 96=72.92$ & $62.5 / 92=67.93$ & $66 / 91=72.52$ \\
\hline Nganasan & & & $63 / 97=64.94$ & $58.5 / 92=63.59$ & $60 / 90=66.66$ \\
\hline Selkup & & & & $68 / 92=73.91$ & $60.5 / 89=67.98$ \\
\hline Kamas & & & & & $63.5 / 85=74.71$ \\
\hline
\end{tabular}

Table 2: The results of the present study

Applying both cladistic procedures, one based on average values (left) and the second on minimum values (right), the following schemes are generated (Diagram D):

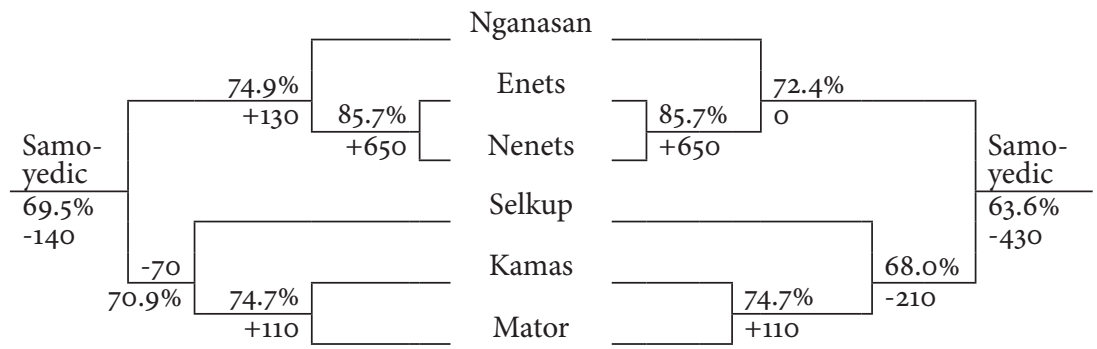

Note: The lexical data of Mator are from the 18th century, whereas the main source of all other wordlists are vocabularies collected by Castrén in the 1840s, supplemented and sometimes verified by lexicons published in the 2oth century. With respect to this fact, the time estimations should be shifted to $c$. the 3 rd century. The question is how much, if lexemes are collected from both older and younger sources in order to obtain a maximal completeness.

Diagram D: The models based on the present results. 
The topologies of the left and right parts of diagram D correspond to one another. In principle, they confirm the north/south dichotomy, proposed in traditional handbooks. With regard to the thin interval $1.4 \%$ between the disintegration of Common Samoyedic and the separation of Selkup from Kamas and Mator, meaning less than plus/minus one cognate item, it is legitimate to approximate the common disintegration around $70 \%$ (c. 100 BCE) for three more or less parallel branches: North Samoyedic, Early Selkup and Kamas-Mator. This alternative conclusion agrees with the left diagram $\mathrm{C}$, based on percentages calculated by Helimski.

Besides the tree diagram, it is useful to depict mutual relations between related languages as a net diagram, shown below in E, where the highest percentages are displayed:

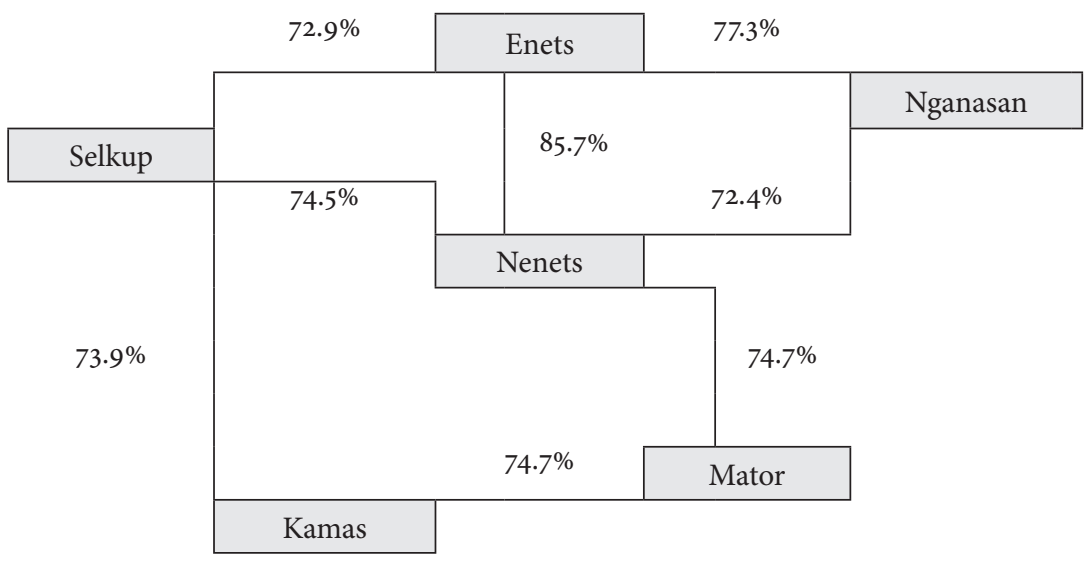

Diagram E: The net diagram connecting the closest relatives

Table 2 shows some apparently deviant results. First of all, the weak links of Nganasan with all other Samoyedic languages with the exception of Enets may be explained by the strong influence of an unidentified pre-Samoyedic substratum of the Taimyr Peninsula, while the higher number of EnetsNganasan cognates was probably caused by their direct contact in the recent past. Similarly, the relatively high scores between Selkup-Nenets and Selkup-Enets can also be ascribed to their neighbourhood existing till the 2oth century. In the 18th century, Kamas and Mator were also spoken in adjoining areas in Southern Siberia (cf. Helimski $1997=$ H: 14-16). Rather surprising is a relatively high score between Nenets and Mator. It could be 
explained by their former neighbourhood. On the other hand, every result operating with Mator (and Kamas) is distorted by the fact that there are 4 missing items and 5 identified loanwords (in Kamas $1+7$, respectively).

The results obtained in the present research should be verified. One of the best tools is analysis of old loanwords, borrowed in this case still before the disintegration of Common Samoyedic. Janhunen (1998: 477) recognized the following old strata of borrowings:

(a) Tocharian ${ }^{1}$ - disintegration c. 400 BCE; hypothetical identification with Afanasievo culture in South Siberia dated to 37th-25th cent. BCE, followed by the culture of Okunevo dated to 25th-19th cent. BCE (Svyatko et al. 2009: 244), formed probably by early Samoyeds (Vadeckaja 1990: 73), and so-called Tarim mummies from c. 2000 BCE (Mallory \& Mair 200o: 314-318; Blažek \& Schwarz 2011: 136-137, 129).

(b) Ugric ${ }^{2}$ - disintegration c. 1480 BCE (Blažek 2012, 34).

(c) Proto-Bolgar ${ }^{3}$ - disintegration of proto-Turkic into proto-Bolgar and the mainstream called Common Turkic is dated to $c .100 \mathrm{BCE}$ or to o by Dybo, depending upon the choice of synonyms (Dybo 2006: 70-771), and to c. 120 BCE by Mudrak (2009: 172-179, see also Schwarz \& Blažek 2011: 31-32).

From these chronological data it is possible to estimate that contacts between ancestors of Samoyeds and Tocharians could have been realized around $2500 \mathrm{BCE}$. On the other hand, it is not necessary to operate with proto-Ugric as a source of Samoyedic ${ }^{\star} n u m$, if the Ob-Ugric disintegration (c. 400 BCE) preceded the disintegration of Samoyedic (c. 140 BCE, or 3 rd century BCE with respect to the times of records). In the case of proto-Bolgar, the situation is opposite: its separation (between 120 BCE and o) follows after the disintegration of Samoyedic (3rd cent. BCE / 16o BCE; other estimations are still deeper in time - see Diagram B right; according to A. Dybo (2006: 783) Selkup separated c. 410 BCE, followed by the North \& South Samoyedic disintegration dated to 140 BCE). This means that the examples quoted by Janhunen as proto-Bolgar (with respect to Samoyedic *jür vs. Volga Bolgar ‘jür, Chuvash śěr, but Common Turkic ^jüz) should be ascribed to proto-Turkic ( ${ }^{*} j \bar{u} \dot{r}$; cf. H 244; Jo 124; Dybo 2006: 786). This conclusion is extraordinarily important in the discussion of the priority of ${ }^{\star} \dot{r}$ (implying secondary 'zetacism') or ${ }^{\star} z$ (implying secondary 'rhotacism') in favour of original ${ }^{\star} r$. Summing up, it is possible to conclude that the disin- 


\section{Václav Blažek}

tegration of Samoyedic in the 3rd-2nd century BCE happened between the disintegrations of its neighbours, Ob-Ugric around 400 BCE and Turkic around 100 BCE.

The Samoyedic homeland is localized by Xelimskij (1989 [2000]: 15-17) in the Southwest Siberian taiga, in the area delimited by a triangle formed by the cities of Tomsk, Krasnoyarsk and Eniseisk. The ancestors of the Selkups moved to the north along the Ob. The North Samoyeds migrated to the north along the Yenisei. The ancestors of Kamas-Koibal tribes moved to the south in the Sayan Mountains, the ancestors of Mator-TaigiKaragas tribes to the east between the Kan and Mana rivers, right tributaries of the Yenisei. All these migrations could have been caused by ancestors of the Yeniseic populations, forming a part of the Hunnic tribal union at that time. Finally, the Yeniseians followed them, perhaps under pressure from the Turkic populations. Janhunen (2009: 72), and subsequently Parpola (2012: 294), identify the proto-Samoyeds with the Tagar bronze age archaeological culture, dated between the 7 th and 3rd century BCE in South Siberia, concretely in the eastern part of Kemerovo province, the southern part of Krasnoyarsk Territory and the Republic of Khakassia.

The percentages of cognates between the individual Samoyedic and Finno-Ugric languages vary between 35\% (Finnish vs. Nganasan, Finnish vs. Kamas) and $18-19 \%$ (North and Inari Saami vs. Selkup; Hungarian vs. Enets). More coherent are the results obtained from average values: maximum 28.62\% of Finnic vs. Samoyedic against 22.39\% of MordvinianSamoyedic and $22.95 \%$ of Saamic-Samoyedic. The average of all averages is c. $25 \%$, corresponding to $3320 \mathrm{BCE}$ (or c. $3400 \mathrm{BCE}$, if the earlier records of some wordlists are taken in account) as a date of disintegration of Uralic into Finno-Ugric and Samoyedic. This agrees better with the dating to 3430 BCE by S. Starostin (2004) than to 3840 BCE, as proposed by his son G. Starostin (p.c., 26 Nov, 2010). 
On the classification of the Samoyedic languages

\section{Statistics}

Missing items: Nenets: ø; Enets: \#\#69, 73; Nganasan: \#73; Kamas: \#73; Mator: \#\#51, 71, 73, 86.

Identified loanwords: Nenets: ø; Enets: ø; Nganasan: \#66; Selkup: \#\#55, 96; Kamas: \#\#1, 6, 8, 27, 35, 69, 100; Mator: \#\#20, 69, 77, 97, 99.

\section{Non-cognates $(\Sigma)$ and shares of common cognates $(\sigma)$ :}

Nenets-Enets: 3 bark, 11 breast, 24 egg, 26 fat, 27 feather, 35 green, 50 louse, 55 mountain, 59 new, 80 star, 83 swim, 84 tail, $85 / 2$ that, $86 / 2$ this, 87 thou; $\Sigma 14 ; \sigma=84 / 98=85.11 \%$.

Nenets-Nganasan: 1 all, 2 ashes, 3 bark, 5 big, 6/2 bird, 8 black, 11 breast, 12 burn, 22 earth, 24 egg, 32 full, 34 good, 35 green, 46 leaf, 49 long, 50 louse, 54 moon, 55 mountain, 59 new, 61 nose, 69 round, 70 sand, 77 small, 80 star, $86 / 2$ this, 87 thou, 93 warm, $95 / 2$ we, $96 / 2$ what; $\sum 27 ; \sigma 71 / 98=72.44 \%$. Enets-Nganasan: 1 all, 2/2 ashes, 5 big, 6 bird, 8 black, 22 earth, 26 fat, 27 feather, 32 full, 34 good, 35 green, 46 leaf, 49 long, 54 moon, 55 mountain, 61 nose, $70 / 2$ sand, 71 say, ?77 small, 83 swim, $85 / 2$ that, $86 / 2$ this, 93 warm, 95/2 we; $\Sigma 21 ; \sigma=76 / 97=78.35 \%$.

Nenets-Selkup: 1 all, 2/2 ashes, 8 black, 11 breast, 24 egg, 26 fat, 31 foot, 32 full, 35 green, 38 head, 47 lie, 50 louse, 51 man, 52 many, 59 new, 64 person, 69 round, 70 sand, 77 small, 80 star, 82 sun, $85 / 2$ that, $86 / 2$ this, 87 thou, 93 warm, 95/2 we, 100 yellow; $\Sigma 25 ; \sigma=73 / 98=74.49 \%$.

Enets-Selkup: 1 all, 2/2 ashes, 4 belly, 6/2 bird, 8 black, 11 breast, 20 dry, 24 egg, 27 feather, 31 foot, 32 full, 38 head, 47 lie, 51 man, 52 many, 58 neck, ?59 new, 64 person, 70 sand, 77 small, 80 star, 82 sun, 83 swim, 84 tail, 93 warm, 95/2 we, 98 who, 100 yellow; $\Sigma 26 ; \sigma=70 / 96=72.92 \%$.

Nganasan-Selkup: 1 all, 2 ashes, 4 belly, 5 big, 6/2 bird, 8 black, 11 breast, 12 burn, 20 dry, 22 earth, 24 egg, 26 fat, 31 foot, 32 full, 34 good, 35 green, 38 head, 46 leaf, 47 lie, 49 long, 51 man, 52 many, 54 moon, 58 neck, 59 new, 61 nose, 64 person, 69 round, $70 / 2$ sand, 71 say, 80 star, 82 sun, ?83 swim, 85/2 that, $86 / 2$ this, $98 / 2$ who, 100 yellow; $\Sigma 34 ; \sigma=63 / 97=64.94 \%$.

Nenets-Kamas: $2 / 2$ ashes, 7 bite, 11 breast, 12 burn, 20 dry, 24 egg, 26 fat, 30 fly, 32 full, 34 good, 38 head, 44 knee, 45 know, 47 lie, 50 louse, 53 meat, 54 moon, 55 mountain, 59 new, 64 person, 66 red, 67 road, 69 round, 70 sand, 77 small, 80 star, 83 swim, 85/2 that, 87 thou, 93 warm, 95/2 we; $\sum 29.5 ; \sigma=$ $62.5 / 92=67.93 \%$. 


\section{Václav Blažek}

Enets-Kamas: $2 / 2$ ashes, 4 belly, 7 bite, 11 breast, 12 burn, 20 dry, 26 fat, 30 fly, 32 full, 34 good, 38 head, 44 knee, 45 know, 47 lie, 53 meat, 54 moon, 55 mountain, 58 neck, 59 new, 64 person, 66 red, 67 road, 70 sand, 77 small, 8 o star, 83 swim, 84 tail, 92 walk, 93 warm, 95/2 we, 98/2 who; $\Sigma 29.5 ; \sigma=$ $62.5 / 92=67.93 \%$.

Nganasan-Kamas: 2 ashes, 5 big, 7 bite, 11 breast, 12 burn, 20 dry, 22 earth, 26 fat, 30 fly, 32 full, 34 good, 38 head, 44 knee, 45 know, 46 leaf, 47 lie, 49 long, 53 meat, 58 neck, 59 new, 61 nose, 64 person, 67 road, 69 round, 70 sand, 71 say, 72 see, 77 small, 80 star, 83 swim, 84 tail, 85/2 that, 93 warm, 96/2 what, 98/2 who; $\sum$ 33.5; $\sigma=58.5 / 92=63.59 \%$.

Selkup-Kamas: 7 bite, ?11 breast, 12 burn, 20 dry, 24 egg, 26 fat, 30 fly, 31 foot, 32 full, 34 good, 44 knee, 45 know, 52 many, 54 moon, 59 new, 64 person, 66 red, 67 road, 69 round, 70 sand, 71 say, 77 small, 82 sun, 93 warm, 98/2who; $\Sigma 24 ; \sigma=68 / 92=73.91 \%$.

Nenets-Mator: $2 / 2$ ashes, 3 bark, 6 bird, 7 bite, 8 black, 11 breast, 24 egg, 32 full, 34 good, 35 green, 46 leaf, 47 lie, 48 liver, 54 moon, 55 mountain, 59 new, 64 person, 67 road, 70 sand, 80 star, $85 / 2$ that, 87 thou, $95 / 2$ we, $96 / 2$ what, 100 yellow; $\sum 23 ; \sigma 68 / 91=74.72 \%$.

Enets-Mator: 1/2 all, 2/2 ashes, 3 bark, 4 belly, 6 bird, 7 bite, 8 black, 24 egg, 26 foot, 27 feather, 32 full, 34 good, 35 green, 46 leaf, 47 lie, 48 liver, 50 louse, 54 moon, 55 mountain, 67 road, 70 sand, 80 star, 83 swim, 84 tail, 95/2 we, 98/2 what, 100 yellow; $\sum 25 ; \sigma=66 / 91=72.52 \%$.

Nganasan-Mator: 1 all, 2 ashes, 3 bark, 4 belly, 5 big, 6 bird, 7 bite, 8 black, 12 burn, 19 drink, 22 earth, 26 fat, 32 full, 34 good, 35 green, 46 leaf, 47 lie, 48 liver, 49 long, 50 louse, 55 mountain, 59 new, 64 person, 67 road, 70 sand, 80 star, 83 swim, 85/2 that, 93 warm, $98 / 2$ who, 100 yellow; $\Sigma 30 ; \sigma=60 / 90$ $=66.66 \%$.

Selkup-Mator: 1 all, 3 bark, 6 bird, 7 bite, 8 black, 11 breast, 19 drink, 24 egg, 26 fat, 31 foot, 32 full, 34 good, 35 green, 38 head, 46 leaf, 47 lie, 48 liver, 50 louse, 52 many, 54 moon, 58 neck, 59 new, 64 person, 67 road, 70 sand, 82 sun, 93 warm, 98/2 who, 100 yellow; $\Sigma 30.5 ; \sigma=60.5 / 89=67.98 \%$.

Kamas-Mator: 3 bark, 7 bite, 11 breast, 12 burn, 19 drink, 32 full, 34 good, 38 head, 44 knee, 45 know, 46 leaf, 47 lie, 48 liver, 50 louse, 53 meat, 58 neck, 59 new, 66 red, 72 see, 83 swim, 92 walk, 96/2 what; $\sum 21.5 ; \sigma=63.5 / 85=74.71 \%$.

Note: The fraction $\mathrm{n} / 2$ is used for partial cognates, e.g. if a word in language A corresponds to a component of a compound in language $\mathrm{B}$, or in the case of pronominal stems agreeing in the initial consonants but differing in the root vowels. 


\section{Samoyedic vs. Finno-Ugric}

\section{Samoyedic vs. Finnic}

\begin{tabular}{|c|c|c|c|c|c|c|c|}
\hline & Nenets & Enets & Nganasan & Selkup & Kamas & Mator & $\varnothing[\%]$ \\
\hline Finnish & $\begin{array}{c}27 / 94= \\
.2872\end{array}$ & $\begin{array}{l}25.5 / 93 \\
=.2742\end{array}$ & $\begin{array}{l}32.5 / 93 \\
=.3495\end{array}$ & $\begin{array}{c}26 / 93= \\
.2796\end{array}$ & $\begin{array}{l}31.5 / 89 \\
=.3539\end{array}$ & $\begin{array}{c}25 / 86= \\
.2907\end{array}$ & 30.57 \\
\hline Karelian & $\begin{array}{c}23 / 92= \\
.2500\end{array}$ & $\begin{array}{c}24 / 91= \\
.2637\end{array}$ & $\begin{array}{c}28 / 92= \\
.3043\end{array}$ & $\begin{array}{c}25 / 91= \\
.2747\end{array}$ & $\begin{array}{c}26 / 87= \\
.2988\end{array}$ & $\begin{array}{c}23 / 84= \\
.2738\end{array}$ & 27.77 \\
\hline Veps & $\begin{array}{l}24.5 / 93 \\
=.2634\end{array}$ & $\begin{array}{c}25 / 92= \\
.2717\end{array}$ & $\begin{array}{c}28 / 93= \\
.3011\end{array}$ & $\begin{array}{l}24.5 / 92 \\
=.2663\end{array}$ & $\begin{array}{c}28 / 88= \\
.3181\end{array}$ & $\begin{array}{l}22.5 / 85 \\
=.2647\end{array}$ & 28.08 \\
\hline Estonian & $\begin{array}{l}24.5 / 93 \\
=.2634\end{array}$ & $\begin{array}{c}26 / 92= \\
.2826\end{array}$ & $\begin{array}{l}29.5 / 92 \\
=.3206\end{array}$ & $\begin{array}{l}25.5 / 92 \\
=.2772\end{array}$ & $\begin{array}{l}27.5 / 88 \\
=.3125\end{array}$ & $\begin{array}{l}23.5 / 85 \\
=.2764\end{array}$ & 28.87 \\
\hline Livonian & $\begin{array}{l}24.5 / 93 \\
=.2634\end{array}$ & $\begin{array}{c}23 / 92= \\
.2500\end{array}$ & $\begin{array}{l}27.5 / 92 \\
=.2989\end{array}$ & $\begin{array}{l}24.5 / 92 \\
=.2663\end{array}$ & $\begin{array}{l}25.5 / 88 \\
=.2897\end{array}$ & $\begin{array}{c}23 / 85= \\
.2706\end{array}$ & 27.31 \\
\hline Votic & $\begin{array}{l}25.5 / 92 \\
=.2772\end{array}$ & $\begin{array}{c}26 / 91= \\
.2857\end{array}$ & $\begin{array}{l}29.5 / 92 \\
=.3206\end{array}$ & $\begin{array}{c}26 / 91= \\
.2857\end{array}$ & $\begin{array}{c}26 / 88= \\
.2954\end{array}$ & $\begin{array}{l}23.5 / 84 \\
=.2798\end{array}$ & 29.09 \\
\hline$\varnothing[\%]$ & $\begin{array}{c}160.46 \\
/ 6= \\
26.74\end{array}$ & $\begin{array}{c}162.79 \\
16= \\
27.12\end{array}$ & $\begin{array}{c}189.50 \\
16= \\
31.58\end{array}$ & $\begin{array}{c}164.98 \\
16= \\
27.50\end{array}$ & $\begin{array}{c}186.84 \\
/ 6= \\
31.14\end{array}$ & $\begin{array}{c}165.60 \\
16= \\
27.60\end{array}$ & $\begin{array}{c}1030.17 \\
/ 36= \\
28.62\end{array}$ \\
\hline
\end{tabular}

\section{Samoyedic vs. Saamic}

\begin{tabular}{|l|c|c|c|c|c|c|c|}
\hline & Nenets & Enets & Nganasan & Selkup & Kamas & Mator & $\varnothing[\%]$ \\
\hline South & $\begin{array}{c}21 / 89= \\
.2360\end{array}$ & $\begin{array}{c}20 / 88= \\
.2273\end{array}$ & $\begin{array}{c}22.5 / 88 \\
=.2557\end{array}$ & $\begin{array}{c}17.5 / 88 \\
=.1989\end{array}$ & $\begin{array}{c}20 / 84= \\
.2380\end{array}$ & $\begin{array}{c}18.5 / 81 \\
=.2284\end{array}$ & 23.07 \\
\hline Lule & $21 / 89=$ & $19 / 88=$ & $22.5 / 88$ & $17.5 / 88$ & $20 / 84=$ & $19.5 / 81$ & 23.09 \\
& .2360 & .2159 & $=.2557$ & $=.1989$ & .2380 & $=.2407$ & \\
\hline North & $20 / 91=$ & $19 / 90$ & $22.5 / 90$ & $16.5 / 90$ & $19 / 85=$ & $19.5 / 83$ & 22.04 \\
& .2198 & $=.2111$ & $=.2500$ & $=.1833$ & .2235 & $=.2349$ & \\
\hline Inari & $20 / 91=$ & $18 / 90=$ & $21.5 / 90$ & $17 / 90=$ & $20 / 85=$ & $18.5 / 83$ & 21.76 \\
& .2198 & .2000 & $=.2389$ & .1889 & .2352 & $=.2229$ & \\
\hline Skolt & $22 / 88=$ & $20 / 87=$ & $23.5 / 87$ & $18 / 87=$ & $21 / 82=$ & $20.5 / 80$ & 24.49 \\
& .2500 & .2299 & $=.2701$ & .2069 & .2560 & $=.2563$ & \\
\hline Kildin & $21 / 88=$ & $19 / 87=$ & $21.5 / 87$ & $17 / 87=$ & $21 / 83=$ & $19.5 / 80$ & 23.27 \\
& .2386 & .2184 & $=.2471$ & .1954 & .2530 & $=.2438$ & \\
\hline Ø [\%] & 140.02 & 130.26 & $151.75 / 6=$ & 117.23 & 144.37 & 142.70 & 826.83 \\
& $16=$ & $/ 6=$ & 25.29 & $/ 6=$ & $16=$ & $16=$ & $/ 36=$ \\
& 23.34 & 21.71 & & 19.54 & 24.06 & 23.78 & 22.95 \\
\hline
\end{tabular}




\section{Václav Blažek}

\section{Samoyedic vs. Mordvin}

\begin{tabular}{|l|c|c|c|c|c|c|c|}
\hline & Nenets & Enets & Nganasan & Selkup & Kamas & Mator & $\varnothing[\%]$ \\
\hline Erzya & $\begin{array}{c}20.5 / 98 \\
=.2092\end{array}$ & $\begin{array}{c}20.5 / 96 \\
=.2135\end{array}$ & $\begin{array}{c}23.5 / 96 \\
=.2448\end{array}$ & $\begin{array}{c}23.5 / 96 \\
=.2448\end{array}$ & $\begin{array}{c}19 / 90 \\
=.2111\end{array}$ & $\begin{array}{c}20.5 / 89 \\
=.2303\end{array}$ & 22.52 \\
\hline Mokša & $\begin{array}{c}20 / 98= \\
.2041\end{array}$ & $\begin{array}{c}20.5 / 96 \\
=.2135\end{array}$ & $\begin{array}{c}22.5 / 96 \\
=.2344\end{array}$ & $\begin{array}{c}23.5 / 96 \\
=.2448\end{array}$ & $\begin{array}{c}19 / 91 \\
=.2111\end{array}$ & $\begin{array}{c}20.5 / 89 \\
=.2303\end{array}$ & 22.26 \\
\hline$[\%]$ & 20.67 & 21.35 & 23.96 & 24.48 & 21.11 & 23.03 & 22.39 \\
\hline
\end{tabular}

\section{Samoyedic vs. Mari}

\begin{tabular}{|l|c|c|c|c|c|c|c|}
\hline Mari & $\begin{array}{c}25.5 / 95.5 \\
=.2578\end{array}$ & $\begin{array}{c}24.5 / 93.5 \\
=.2620\end{array}$ & $\begin{array}{c}23.5 / 93.5 \\
=.2513\end{array}$ & $\begin{array}{c}24 / 93.5 \\
=.2567\end{array}$ & $\begin{array}{c}23 / 88.5 \\
=.2598\end{array}$ & $\begin{array}{c}23.5 / 86.5 \\
=.2717\end{array}$ & 26.12 \\
\hline$\varnothing[\%]$ & 25.78 & 26.20 & 25.13 & 25.67 & 25.98 & 27.17 & 26.12 \\
\hline
\end{tabular}

\section{Samoyedic vs. Permic}

\begin{tabular}{|l|c|c|c|c|c|c|c|}
\hline & Nenets & Enets & Nganasan & Selkup & Kamas & Mator & $\varnothing[\%]$ \\
\hline Udmurt & $\begin{array}{c}25 / 97= \\
.2578\end{array}$ & $\begin{array}{c}23 / 95= \\
.2316\end{array}$ & $\begin{array}{c}26.5 / 95 \\
=.2789\end{array}$ & $\begin{array}{c}23.5 / 95 \\
=.2474\end{array}$ & $\begin{array}{c}25 / 89= \\
.2808\end{array}$ & $\begin{array}{c}24.5 / 88 \\
=.2784\end{array}$ & 26.26 \\
\hline Zyryan & $\begin{array}{c}26 / 98 \\
=.2693\end{array}$ & $\begin{array}{c}23 / 96= \\
.2396\end{array}$ & $\begin{array}{c}25.5 / 96 \\
=.2656\end{array}$ & $\begin{array}{c}24 / 96 \\
=.2500\end{array}$ & $\begin{array}{c}25 / 96= \\
.2604\end{array}$ & $\begin{array}{c}24.5 / 90 \\
=.2722\end{array}$ & 26.12 \\
\hline Permiac & $\begin{array}{c}25 / 95= \\
.2632\end{array}$ & $\begin{array}{c}23 / 93= \\
.2473\end{array}$ & $\begin{array}{c}24.5 / 93 \\
=.2634\end{array}$ & $\begin{array}{c}21.5 / 93 \\
=.2312\end{array}$ & $\begin{array}{c}24 / 89 \\
=.2696\end{array}$ & $\begin{array}{c}22.5 / 88 \\
=.2557\end{array}$ & 25.46 \\
\hline$\varnothing[\%]$ & $79.03 / 3$ & $\begin{array}{c}71.85 / 3 \\
=23.95\end{array}$ & $\begin{array}{c}80.79 / 3 \\
=26.93\end{array}$ & $\begin{array}{c}72.86 / 3 \\
=24.29\end{array}$ & $\begin{array}{c}81.08 / 3 \\
=27.02\end{array}$ & $\begin{array}{c}80.63 / 3 \\
=26.88\end{array}$ & $\begin{array}{c}155.70 / 6 \\
=25.95\end{array}$ \\
\hline
\end{tabular}

\section{Samoyedic vs. Ugric}

\begin{tabular}{|c|c|c|c|c|c|c|c|}
\hline & Nenets & Enets & Nganasan & Selkup & Kamas & Mator & $\varnothing[\%]$ \\
\hline Hungarian & $\begin{array}{l}19.5 / 95 \\
=.2053\end{array}$ & $\begin{array}{l}17.5 / 93 \\
=.1882\end{array}$ & $\begin{array}{c}21 / 93= \\
.2258\end{array}$ & $\begin{array}{c}20 / 93 \\
=.2151 \\
\end{array}$ & $\begin{array}{c}22 / 88= \\
.2500\end{array}$ & $\begin{array}{l}20.5 / 89 \\
=.2356\end{array}$ & 22.00 \\
\hline Mansi N. & $\begin{array}{l}23.5 / 99 \\
=.2374\end{array}$ & $\begin{array}{c}21 / 98= \\
.2143\end{array}$ & $\begin{array}{c}25 / 98= \\
.2551\end{array}$ & $\begin{array}{l}23.5 / 98 \\
=.2398\end{array}$ & $\begin{array}{l}22.5 / 92 \\
=.2445\end{array}$ & $\begin{array}{c}24 / 91= \\
.2637\end{array}$ & 24.24 \\
\hline Mansi E. & $\begin{array}{l}23 / 100 \\
=.2300\end{array}$ & $\begin{array}{l}20.5 / 98 \\
=.2092\end{array}$ & $\begin{array}{c}24 / 98= \\
.2449\end{array}$ & $\begin{array}{c}24 / 98= \\
.2449\end{array}$ & $\begin{array}{c}22 / 92= \\
.2391\end{array}$ & $\begin{array}{l}22.5 / 91 \\
=.2473\end{array}$ & 23.59 \\
\hline Khanty S. & $\begin{array}{c}25 / 99= \\
.2525\end{array}$ & $\begin{array}{c}21 / 97= \\
.2165\end{array}$ & $\begin{array}{c}25 / 97= \\
.2577\end{array}$ & $\begin{array}{l}26.5 / 97 \\
=.2732 \\
\end{array}$ & $\begin{array}{l}23.5 / 92 \\
=.2554\end{array}$ & $\begin{array}{c}25 / 90= \\
.2778\end{array}$ & 25.55 \\
\hline Khanty E. & $\begin{array}{c}23 / 99= \\
.2323\end{array}$ & $\begin{array}{c}21 / 97= \\
.2165\end{array}$ & $\begin{array}{c}24 / 97= \\
.2474\end{array}$ & $\begin{array}{c}26 / 97= \\
.2680\end{array}$ & $\begin{array}{l}23.5 / 92 \\
=.2554\end{array}$ & $\begin{array}{c}24 / 90= \\
.2667\end{array}$ & 24.76 \\
\hline$\varnothing[\%]$ & $\begin{array}{l}115.75 / 5 \\
=23.15\end{array}$ & $\begin{array}{c}104.47 / 5 \\
=20.89\end{array}$ & $\begin{array}{l}123.09 / 5 \\
=24.62\end{array}$ & $\begin{array}{c}124.10 / 5 \\
=24.82\end{array}$ & $\begin{array}{c}124.44 / 5 \\
=24.88\end{array}$ & $\begin{array}{l}129.11 / 5 \\
=25.82\end{array}$ & $\begin{array}{c}720.96 \\
130= \\
24.03\end{array}$ \\
\hline
\end{tabular}

$\varnothing \emptyset[\%](28.62+22.95+22.39+26.12+25.95+24.03) / 6=150.06 / 6=25.01 \%$ 
On the classification of the Samoyedic languages

\section{Tree diagram of the disintegration of 28 Uralic languages in the light of absolute chronology}

\begin{tabular}{|c|c|c|c|c|c|c|}
\hline-2500 & 1 & 1500 & -500 & +1500 & +150 \\
\hline
\end{tabular}

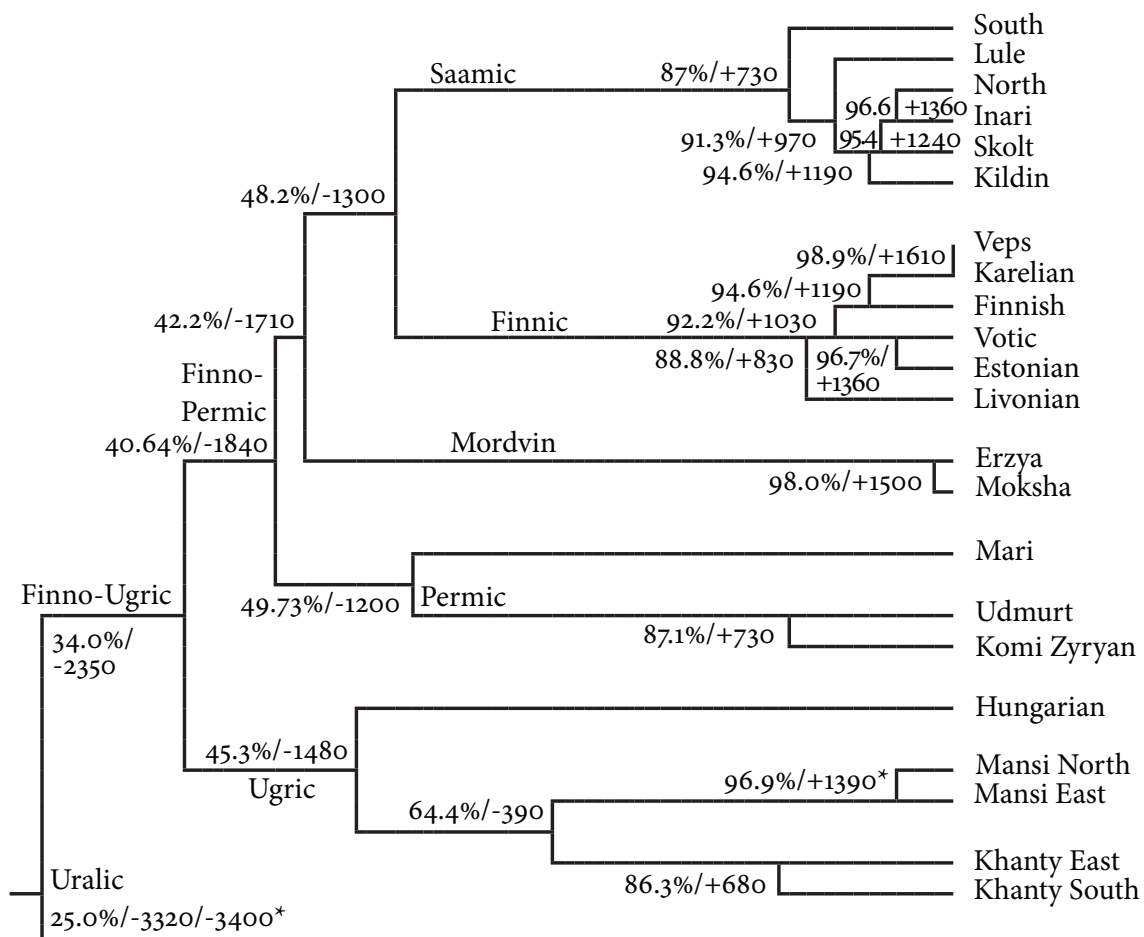

$25.0 \% /-3320 /-3400^{*}$

Nganasan

Enets

Nenets

Samoyedic

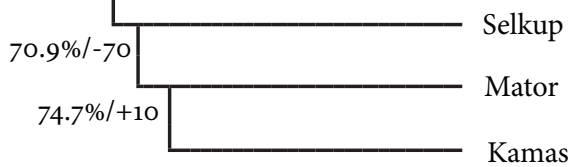

$-2500$

$-1500$

$-500$

$+500$

$+1500$ 


\section{Václav Blažek}

* Note: The lexical data of Mordvin and Mansi were collected at the end of the 19th century by Heikki Paasonen and Bernát Munkácsi, respectively, so the calculated dating of disintegration should be postponed $c$. one century in the past. Similarly, the lexical data of Mator are from the 18th century, while the main source of all other Samoyedic wordlists are vocabularies collected by M. A. Castrén in the 1840s, supplemented and sometimes verified by lexicons published in the 2oth century. With respect to this fact, the time estimation of the Samoyedic dispersion should be shifted to the 3 rd century BCE. The question is how much, if lexemes are collected from both older and younger sources in order to obtain a maximal completeness. Finally, the beginning of disintegration of the Uralic protolanguage should be shifted to $c .3400$ BCE.

\section{Wordlists with etymological comments}

\begin{tabular}{|c|c|c|c|c|c|c|c|}
\hline gloss & Nenets & Enets & Nganasan & Selkup & Kamas & Mator & etymology \\
\hline 1a. all & tuku' & tuku' & & & & & $\begin{array}{l}{ }^{*} t \ddot{u ̈ t} t_{3} \sim{ }^{*} t \ddot{u} k- \\
(J 168)\end{array}$ \\
\hline laa. & $\begin{array}{l}\text { tübea }{ }^{\text {Knd. }} \\
\text { cf. tjuki- } \\
\text { peäj }\end{array}$ & & & & & tupuj $j^{\mathrm{Mt}}$ & 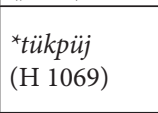 \\
\hline $1 \mathrm{~b}$. & nokxa $a_{\mathrm{RNS}}$ & & & & & & $\begin{array}{l}\text { cf. nokava } \\
\text { majority }\end{array}$ \\
\hline $1 c$. & $\mathrm{mal}_{\mathrm{RNS}}$ & & & & & & cf. mal end \\
\hline $1 \mathrm{~d}$. & & kurhari $_{\mathrm{M}}$ & & & & & $\begin{array}{l}\text { cf. kuraha } \\
\text { wie }_{\mathrm{Pr}_{\mathrm{r}}}\end{array}$ \\
\hline le. & & & bansa & & $\begin{array}{l}\text { (buušša } \\
\text { heil, ganz) }\end{array}$ & & $\begin{array}{l}\mathrm{U}^{*} \text { weńćc } \\
(\mathrm{R} 568)\end{array}$ \\
\hline 1f. & & & & $\begin{array}{l}\text { mûn- } \\
d e \eta^{\mathrm{B} \text {,Tas }}\end{array}$ & & & \\
\hline $1 \mathrm{~g}$. & & & & sielay $^{\mathrm{OO}, \mathrm{C}}$ & & & \\
\hline 1h. & & & & $w \underline{s}^{\mathrm{MO}, \mathrm{K}, \mathrm{NP}}$ & & & $<\mathrm{Ru} v e s ́$ \\
\hline 1i. & & & & & $\underline{b a r}=\underline{b \bar{a}} r_{D}$ & & $\begin{array}{l}<\text { Tat } b \bar{a} r \text { id., } \\
\text { whole }\end{array}$ \\
\hline $\begin{array}{l}\text { a. } \\
\text { ashes }\end{array}$ & tuija & & & & & & $\begin{array}{l}\text { cf. }{ }^{*} t u j \text { fire } \\
\text { (J 166) }\end{array}$ \\
\hline $2 \mathrm{ab}$. & $\begin{array}{l}\text { tunzibea } \\
\text { tu's sibja }\end{array}$ & \begin{tabular}{|l|}
$t u s^{\mathrm{B}}$ \\
$t u ̄ s i i^{\mathrm{Ch}}$ \\
$(\mathrm{M} 205$, \\
$227)$ \\
\end{tabular} & $\begin{array}{l}(\operatorname{simi} \\
\text { coal })\end{array}$ & $\begin{array}{l}\text { šiu }{ }^{\mathrm{O}} \\
\text { sîwa } \\
\text { šime } \\
\text { sias, B }\end{array}$ & $\begin{array}{l}\text { šünö } \\
\text { šymy }\end{array}$ & giima $^{\mathrm{Kg}}$ & $\begin{array}{l}{ }^{*} \text { kimä }(\mathrm{J} \\
70)= \\
{ }^{*} \text { kime }(\mathrm{H} \\
487)\end{array}$ \\
\hline $2 c$. & & & $\begin{array}{l}j u a \\
(+ \text { sand })\end{array}$ & & & & $\begin{array}{l}\text { *jåô (J 36- } 37)=\# 22\end{array}$ \\
\hline $2 \mathrm{cc}$. & & jâra & & & & & $\begin{array}{l}\text { *jåôråå (J 37) } \\
-\# 70\end{array}$ \\
\hline
\end{tabular}


On the classification of the Samoyedic languages

\begin{tabular}{|c|c|c|c|c|c|c|c|}
\hline gloss & Nenets & Enets & Nganasan & Selkup & Kamas & Mator & etymology \\
\hline $2 \mathrm{~d}$. & (tab sand) & $\begin{array}{l}\text { (tóbo } \\
\text { clay }= \\
\text { tobo } \breve{\mathrm{Te}}_{\mathrm{Te}} \\
\text { sand) }\end{array}$ & & & & $\begin{array}{l}t \bar{a}^{\mathrm{Mt}} \\
\left(\text { taha } a^{\mathrm{Kg}}\right. \\
\operatorname{dirt})\end{array}$ & $\begin{array}{l}{ }^{*} t_{1} \text { âpâ (J 148; } \\
\text { H 937) }\end{array}$ \\
\hline $2 e$. & & & & püs ${ }^{1} u^{\mathrm{MK}} /$ & & & \\
\hline $2 f$. & & & & $k^{2} u \hat{n} n \partial^{\mathrm{MK} /}$ & & & \\
\hline $2 \mathrm{~g}$. & & & & $k^{\prime}{ }^{\prime} r a^{\mathrm{Tu}} /{ }_{\mathrm{DA}}$ & & & cf. \#70: sand \\
\hline $2 \mathrm{~h}$. & & & & $k \bar{a} n t \underline{a}^{\mathrm{Tu}}{ }_{\mathrm{DA}}$ & & & $<$ Ket xonti \\
\hline $2 \mathrm{i}$. & & & & & $\underline{k}^{\prime} i^{\prime} \varepsilon_{\mathrm{D}}$ & & $\begin{array}{l}\text { cf. Tk kömür } \\
\text { id. }\end{array}$ \\
\hline $\begin{array}{l}3 a . \\
\text { bark }\end{array}$ & pea hôba & & & $\begin{array}{l}\text { pôt kôba- } \\
\mathrm{N}, \mathrm{K}\end{array}$ & & & $\begin{array}{l}{ }^{*} k o p a ̊(\mathrm{~J} \mathrm{73}) \\
-\# 75 \\
<\mathrm{U}{ }^{\star} k o p a \\
(\mathrm{R} 180)= \\
{ }^{*} k o p a ̊\left(\mathrm{~S}_{1}\right. \\
537)\end{array}$ \\
\hline $3 b$. & seabt/t & & & & & & \\
\hline $3 c$. & pir & & & & pere $e_{\text {Klaproth }}$ & & $\begin{array}{l}<\mathrm{U}^{*} \text { per3 } \\
(\mathrm{R} 374)= \\
{ }^{*} \text { peri }\left(\mathrm{S}_{1} 539\right)\end{array}$ \\
\hline $3 \mathrm{~d}$. & & $\begin{array}{l}\text { sésa } a^{\mathrm{Ch}} \\
\theta e \theta a^{\mathrm{B}} / \mathrm{Gl}\end{array}$ & kasu & $k \hat{a} s^{\mathrm{NP}, \mathrm{B}, \mathrm{Tas}}$ & $\begin{array}{l}k a z a= \\
k^{\prime} a z a{ }_{D}\end{array}$ & & $\begin{array}{l}{ }^{*} k a ̈ s a ̊(J ~ 65) \\
<\mathrm{U} \\
{ }^{*} k o(n){ }^{\prime} c_{k 3} \\
(\mathrm{R} 179)\end{array}$ \\
\hline $3 e$. & & & & & & tebena & 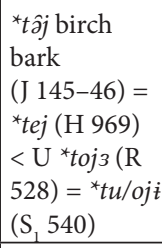 \\
\hline $\begin{array}{l}\text { 4a. } \\
\text { belly }\end{array}$ & $\begin{array}{l}\text { ńanko }_{\mathrm{RNS}} \\
\text { (ńân } \\
\text { Lab- } \\
\text { magen) }\end{array}$ & & & 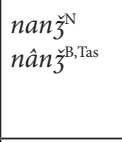 & nâna & $\begin{array}{l}\text { aehnde } \\
\text { énde }\end{array}$ & $\begin{array}{l}{ }^{*} \ddot{a} n c \hat{\partial}(\mathrm{J} \mathrm{20})= \\
*(n) \ddot{a} n c a \\
(\mathrm{H} \mathrm{73})\end{array}$ \\
\hline $4 \mathrm{~b}$. & $\begin{array}{l}m y^{\prime}= \\
m y^{\prime}(n)_{\mathrm{RNS}}\end{array}$ & \begin{tabular}{|l} 
munori $^{\mathrm{Ch}}$ \\
munedi $^{\mathrm{B}}$ \\
\end{tabular} & minadâ & & & & $\begin{array}{l}{ }^{*} \text { min- } \\
\text { (JN 175) }\end{array}$ \\
\hline $4 c$. & & $\begin{array}{l}\text { (fediko } \\
\text { Fischma- } \\
\text { gen) }\end{array}$ & & $\begin{array}{l}\text { pärg }^{\mathrm{N}, \mathrm{B}} \\
\text { perge }^{\mathrm{Tas}}\end{array}$ & & $\begin{array}{l}\text { hergè }^{\mathrm{Kg}} \\
\text { hérgem }^{\mathrm{Tg}}\end{array}$ & $\begin{array}{l}{ }^{*} \text { perkä (J 122; } \\
\text { H 284; } \\
\text { R 378-79: } \\
\text { U *pijra) }\end{array}$ \\
\hline $4 \mathrm{~d}$. & & $\begin{array}{l}\text { suse } \mathrm{B}^{\mathrm{B}} \\
\text { sude } e^{\mathrm{Ch}} \\
\text { (+ Inne- } \\
\text { res) }\end{array}$ & & & & & $\begin{array}{l}\text { cf. } 11 \mathrm{~b} \\
\mathrm{U}{ }^{*} \text { śińćäa } \\
(\mathrm{R} 480)\end{array}$ \\
\hline
\end{tabular}


Václav Blažek

\begin{tabular}{|c|c|c|c|c|c|c|c|}
\hline gloss & Nenets & Enets & Nganasan & Selkup & Kamas & Mator & etymology \\
\hline $4 \mathrm{e}$. & $t i \bar{w} w_{\mathrm{NRS}}$ & & $\begin{array}{l}\text { čajbo } \partial_{\mathrm{NRN}} \\
\text { (tajbajara } \\
\text { stomach) }\end{array}$ & \begin{tabular}{|l}
$\left(\check{c} a ̊ t^{\mathrm{Tas}}\right.$ \\
content of \\
stomach $)$
\end{tabular} & šuuia & & $\begin{array}{l}<\mathrm{U}{ }^{*} \text { ćowja / } \\
{ }^{*} \text { cojwa }(\mathrm{R} 40)\end{array}$ \\
\hline $4 \mathrm{f}$. & & & & $\begin{array}{l}\text { kegm- } \\
\check{c} \mathrm{~g}^{\mathrm{Tym}} /{ }_{\mathrm{DA}}\end{array}$ & & & \\
\hline $4 \mathrm{~g}$. & & & & $\frac{\underline{\operatorname{simm}}-}{\underline{\partial t^{\mathrm{Tym}} /}}$ & & & $<\mathrm{Ru}$. život \\
\hline 5a. big & ya/âr(ka) & $\begin{array}{l}\text { arikeo }^{\mathrm{B}} \\
\text { atikid'u }^{\mathrm{Ch}}\end{array}$ & & $\begin{array}{l}\operatorname{warg}^{\mathrm{N}}, \\
\text { warga }^{\mathrm{K}, \mathrm{OO}, \mathrm{C}}, \\
\text { wuerg }^{\mathrm{Tas}},\end{array}$ & $\begin{array}{l}\text { urgo }= \\
\text { uryo }_{\mathrm{D}}\end{array}$ & $\begin{array}{l}\operatorname{orga}^{\mathrm{Mt}} \\
\operatorname{argó}^{\mathrm{Tg}}\end{array}$ & $\begin{array}{l}\text { *årkå : *årâ } \\
\text { greatness } \\
\text { (J 19; H 816) }\end{array}$ \\
\hline $5 b$. & & & anie & & & & $\begin{array}{l}<\mathrm{U}^{*} \text { enä } \\
(\mathrm{R} 74-75): \\
\mathrm{FU}{ }^{*} e / \text { inä } \\
(\mathrm{S}, 541)\end{array}$ \\
\hline $5 c$. & & & $m \partial \eta \partial \delta \partial_{\mathrm{NRN}}$ & & & & \\
\hline $\begin{array}{l}\text { 6a. } \\
\text { bird }\end{array}$ & \begin{tabular}{|l} 
tìrta \\
sârmik \\
flying \\
animal
\end{tabular} & $\begin{array}{l}\text { sáma } \\
(=\text { sâme } \\
\text { wolla })\end{array}$ & & \begin{tabular}{|l} 
tild’eres \\
-surêm \\
telTas \\
telžr- \\
hûrup \\
flying \\
animal \\
\end{tabular} & & $\begin{array}{l}\left(\text { sarma }^{\mathrm{Kg}}\right. \\
\text { Terao } \\
\text { Bonasia) }\end{array}$ & $\begin{array}{l}{ }^{*} \text { sårmå (J } \\
136 ; \text { H 853)< } \\
\text { U *śurme } \\
\text { (R 490-91) }\end{array}$ \\
\hline $6 \mathrm{~b}$. & & & $\begin{array}{l}\text { d'amady- } \\
\text { tyjríe }_{\text {Sim }}\end{array}$ & & & & (X 132) \\
\hline $6 c$. & & & taibaว $\partial_{\mathrm{NRN}}$ & & & & cf. tajbu tail \\
\hline $6 \mathrm{~d}$. & & & & & kuśs & & $\begin{array}{l}<\text { Sag kus }< \\
\text { Tk *kuš (Rä } \\
305)\end{array}$ \\
\hline $6 e$. & & & & & & morena $^{\mathrm{Kg}}$ & (H 692) \\
\hline $6 f$. & & & & & & $\begin{array}{l}\text { tčindžja- } \\
x a^{\mathrm{Mt}} \\
\text { sinschá- } \\
k u^{\mathrm{Tg}, \mathrm{Kg}}\end{array}$ & $\begin{array}{l}{ }^{*} s i_{1} n s a_{1} k k_{3} \\
(\mathrm{H} 889 ; \mathrm{J} \\
141)<\mathrm{U} c ́[i] \\
\eta c_{3}\left(-k_{3}\right)(\mathrm{R} \\
48)\end{array}$ \\
\hline $\begin{array}{l}\text { 7a. } \\
\text { bite }\end{array}$ & seakalyâu & $\begin{array}{l}\text { sektuc }^{\check{B}^{\mathrm{B}}} \\
\text { sckutuya }^{\mathrm{Ch}}\end{array}$ & $\begin{array}{l}\text { sakala- } \\
s a_{\mathrm{NRN}}\end{array}$ & \begin{tabular}{|l|} 
sâžam \\
seačam $^{\mathrm{B}, \mathrm{Ta}}$ \\
hâžap \\
\end{tabular} & & & ${ }^{*}$ säc- (J 136) \\
\hline $7 \mathrm{~b}$. & & & & & thalbelim & & \\
\hline $7 \mathrm{c}$. & & & & & lậilem $_{\mathrm{D}}$ & & \\
\hline $7 \mathrm{~d}$. & & & & & t'udōlāam $m_{\mathrm{D}}$ & & $\begin{array}{l}{ }^{*} t_{3} t_{3} w a- \\
\text { chew }(\mathrm{H} \\
1039)\end{array}$ \\
\hline $7 e$. & & & & & & $\begin{array}{l}\text { balija- } \\
m v^{\mathrm{Mt}}\end{array}$ & $\begin{array}{l}{ }^{*} \text { môls- break } \\
(\mathrm{J} 86 ; \mathrm{H} 97)< \\
\mathrm{U}^{*} \text { mols } \\
\text { (R 278) }\end{array}$ \\
\hline
\end{tabular}


On the classification of the Samoyedic languages

\begin{tabular}{|c|c|c|c|c|c|c|c|}
\hline gloss & Nenets & Enets & Nganasan & Selkup & Kamas & Mator & etymology \\
\hline $\begin{array}{l}8 \mathrm{a} . \\
\text { black }\end{array}$ & paridie & $\begin{array}{l}\text { forededde } \\
\text { forered- } \\
\text { de } \\
\end{array}$ & & & & & cf. $12 \mathrm{a}$ \\
\hline $8 \mathrm{~b}$. & & $\begin{array}{l}\text { loggedded- } \\
d e^{\mathrm{Ch}}\end{array}$ & & & & & \\
\hline $8 c$. & & $\begin{array}{l}\text { toggoéd- } \\
d e^{\mathrm{Ch}} \\
(+ \text { blue })\end{array}$ & & & & & \\
\hline $8 \mathrm{~d}$. & & & $\begin{array}{l}\text { feanka }= \\
\text { heyk } \partial\end{array}$ & & & & \\
\hline $8 \mathrm{e}$. & & & $\begin{array}{l}\text { tusaj- } \\
\text { kuo }_{\mathrm{NRN}}\end{array}$ & & & & \\
\hline $8 f$. & & & & 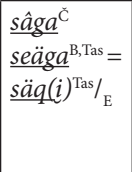 & $\begin{array}{l}\underline{s \hat{a} g a r}= \\
\underline{s \bar{a}(\gamma \hat{a}) r_{D}}\end{array}$ & & $\begin{array}{l}\text { < Tn: Ew } \\
\text { saha grey, } \\
\text { sakarin } \text { black } \\
\text { (DA 2618; } \\
\text { TMS II, 56) }\end{array}$ \\
\hline $8 \mathrm{~g}$. & & & & $\begin{array}{l}\text { tāto- } \\
\text { rul }^{\mathrm{UK} / \mathrm{DA}}\end{array}$ & & & \\
\hline $8 \mathrm{~h}$. & & & & & & küntüh $\ddot{a}^{\mathrm{Mt}}$ & 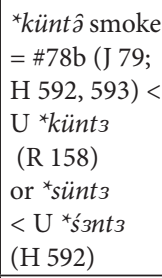 \\
\hline $\begin{array}{l}\text { 9a. } \\
\text { blood }\end{array}$ & $\begin{array}{l}\text { hêm, } \\
\text { xeam }\end{array}$ & $k \imath^{\mathrm{C} h}, k i^{\mathrm{B}}$ & $\mathrm{kam}$ & $\begin{array}{l}k \dot{e} m^{\mathrm{B}, \mathrm{Tas}} \\
{k a m^{\mathrm{K}}}, \\
k_{a} \mathrm{p}^{\mathrm{N}}\end{array}$ & $\begin{array}{l}\text { khem }= \\
k^{\prime} \partial m / k \partial m_{\mathrm{D}}\end{array}$ & $k e m v^{\mathrm{Mt}}$ & $\begin{array}{l}{ }^{\star} k e(\hat{\partial}) m \\
(\mathrm{~J} \mathrm{65)} \\
{ }^{*} \mathrm{kem}(\mathrm{H} 477)\end{array}$ \\
\hline $9 b$. & $\underline{v e j a_{\mathrm{RNS}}}$ & $\frac{\text { buija }}{45)}^{\mathrm{B}}(\mathrm{M}$ & & & & & $\begin{array}{l}{ }^{*} \text { wejå }<\mathrm{Tn} \\
{ }^{*} \text { böjö-kse } \\
(\mathrm{AX} 109)\end{array}$ \\
\hline $9 \mathrm{c}$. & & & demoz ${ }_{N R N}$ & & & & \\
\hline $\begin{array}{l}\text { 10a. } \\
\text { bone }\end{array}$ & $l y, l e^{\prime}$ & tiri ${ }^{\mathrm{Ch}}, \not i d i^{\mathrm{B}}$ & latâ & $\begin{array}{l}l y^{\mathrm{B}}, l \ddot{a}^{\mathrm{OO}}, \\
l i^{\mathrm{N}}\end{array}$ & le & $l e^{\mathrm{Mt}}$ & $\begin{array}{l}*{ }^{* l e}(\mathrm{~J} 82 ; \mathrm{H} \\
618)<\mathrm{U} \\
* l u w e \\
(\mathrm{R} 254-55)= \\
* \operatorname{lix} \dot{(S}, 538)\end{array}$ \\
\hline $\begin{array}{l}11 \mathrm{a} . \\
\text { breast }\end{array}$ & leambara & & & $\begin{array}{l}(l a ̂ m b a ̂ r- \\
l a_{\mathrm{D}} \text { lower } \\
\text { ribs })\end{array}$ & & & $\begin{array}{l}\text { *lempårå } \\
\text { (J 82) }\end{array}$ \\
\hline $11 \mathrm{~b}$. & $\begin{array}{l}\left(\operatorname{siun} z_{\mathrm{NRN}}\right. \\
\text { youngest } \\
\text { child) }\end{array}$ & $\begin{array}{l}\text { śudo }^{\mathrm{Ch}} \\
\text { śuso }^{\mathrm{B}}\end{array}$ & sinśa & & & $\begin{array}{l}\text { künd- } \\
\text { schu }{ }^{\mathrm{Mt}} \\
\text { kûn- } \\
\text { schum }^{\mathrm{Tg}}\end{array}$ & 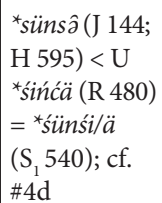 \\
\hline
\end{tabular}


Václav Blažek

\begin{tabular}{|c|c|c|c|c|c|c|c|}
\hline gloss & Nenets & Enets & Nganasan & Selkup & Kamas & Mator & etymology \\
\hline $11 \mathrm{c}$. & & $u i^{\mathrm{B}}, \hat{u}^{\mathrm{Ch}}$ & & & & & $\begin{array}{l}\text { : uinado, } \\
\text { ûyaro }^{\mathrm{Ch}} \\
\text { saugen }(\mathrm{M} \\
230)\end{array}$ \\
\hline $11 \mathrm{~d}$. & & & ńimín & & & & $\begin{array}{l}\text { * ńimmä milk } \\
:{ }^{*} \text { ńim- suck } \\
(\mathrm{J} 110-11)< \\
\mathrm{U}{ }^{*} \text { ime } \\
(\mathrm{R} \text { 82-83) = } \\
{ }^{*} \text { imi- }(\mathrm{S}, 536)\end{array}$ \\
\hline $11 \mathrm{e}$. & & & & \begin{tabular}{|l|}
$k y l^{\mathrm{B}}$ \\
kyle $^{\mathrm{NP}}$ \\
\end{tabular} & & & $11 \mathrm{f} ?$ \\
\hline $11 f$. & & & & & \begin{tabular}{|l} 
khüjüu = \\
$k^{c} u i \partial_{0}$ \\
\end{tabular} & & $11 \mathrm{e} ?$ \\
\hline $11 \mathrm{~g}$. & & & & & numia & & \\
\hline $\begin{array}{l}12 \mathrm{a} . \\
\text { burn } \\
\text { tr. }\end{array}$ & parâdm & $\begin{array}{l}\text { poras }^{\mathrm{K}} / \mathrm{M} \\
\text { poraj }^{\mathrm{B}} / \mathrm{Te} \\
(\text { forará- } \\
\text { bo }^{\mathrm{Ch}} \\
\text { foradabo }^{\mathrm{B}} \\
\text { braten) } \\
\end{array}$ & & $\begin{array}{l}\text { porruay }^{\mathrm{NP}} \\
\text { porua- } \\
\eta^{\text {č,oo }}\end{array}$ & $\begin{array}{l}\text { (para- } \\
\text { bake } \\
\text { \{or better } \\
\text { to } p \ddot{u}- \\
\text { ripen }\})\end{array}$ & $\begin{array}{l}\text { korom- } \\
- \text { namb }^{\mathrm{Mt}} \\
(=x-)\end{array}$ & $\begin{array}{l}\text { *pârå- (J 114; } \\
\text { cf. M 95) < U } \\
{ }^{*} \text { prr3 } \\
(\mathrm{R} 415)\end{array}$ \\
\hline $12 \mathrm{~b}$. & $\begin{array}{l}\text { (lejo } \\
\text { flame) }\end{array}$ & $\begin{array}{l}\text { loirebo } \\
\text { loireo }^{\mathrm{B}}= \\
\text { leidede }_{\mathrm{x}}\end{array}$ & $\begin{array}{l}\text { loyúdi } i_{\mathrm{X}}= \\
\text { layudi }{ }_{\mathrm{NRN}}\end{array}$ & & & & $\begin{array}{l}\text { *lejg } \partial / \text { å- } \\
\text { (JN 173) }\end{array}$ \\
\hline $12 \mathrm{c}$. & & & & $\begin{array}{l}\underline{c} a \bar{p}(p)- \\
i q o^{\text {Tas }} / \mathrm{E}\end{array}$ & & & \\
\hline $12 \mathrm{~d}$. & & & & & neńiläm & & \\
\hline $12 \mathrm{e}$. & & & & & amōlảm $_{\mathrm{D}}$ & & \\
\hline $\begin{array}{l}\text { 13a. } \\
\text { claw } \\
\text { finger- } \\
\text { nail }\end{array}$ & hada & $\begin{array}{l}\text { kora }^{\mathrm{Ch}} \\
\text { koda }^{\mathrm{B}}\end{array}$ & katu & 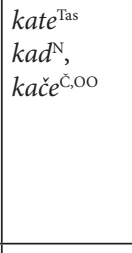 & $\begin{array}{l}\text { kata } \\
\text { k’åda. }_{\mathrm{D}} \\
<\text { Tat, Soy } \\
\text { qadaq } \\
\text { Nagel }\end{array}$ & $\begin{array}{l}k a d a-m v^{\mathrm{Mt}} \\
x a ́ d a-d a^{\mathrm{Kg}}\end{array}$ & 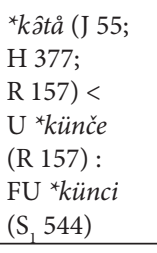 \\
\hline $13 b$ & & & & $\mid \begin{array}{l}\frac{k \ddot{u} \partial-}{l a ̈ s}{ }^{\mathrm{Ty}} /{ }_{\mathrm{DA}} \\
\text { claw }\end{array}$ & & & $<$ Ket qoleś \\
\hline $\begin{array}{l}14 \mathrm{a} . \\
\text { cloud }\end{array}$ & tî̀, tir & $\begin{array}{l}\text { tioti }^{\mathrm{Ch}} \\
\text { tiori }^{\mathrm{B}} \\
\end{array}$ & tî̀u & $\begin{array}{l}t \hat{\imath} t t e^{\mathrm{B}, \mathrm{Tas}} \\
t i t^{\mathrm{N}, \mathrm{B}, \mathrm{Tas}}\end{array}$ & $\begin{array}{l}t i_{\text {Adelung }}= \\
\check{c} i_{\mathrm{D}} \\
\end{array}$ & $\begin{array}{l}t i^{\mathrm{Mt}} \\
d i^{\mathrm{Kg}} \\
\end{array}$ & $\begin{array}{l}{ }^{*} t i a ̂(J ~ 162 ; \\
\text { H 1008) }\end{array}$ \\
\hline $14 \mathrm{~b}$. & & & & $\begin{array}{l}\text { nut mar- } \\
k \mathrm{~kg}^{\text {Tas }} / \mathrm{E} \\
\text { bush of } \\
\text { sky }\end{array}$ & & & \\
\hline
\end{tabular}


On the classification of the Samoyedic languages

\begin{tabular}{|c|c|c|c|c|c|c|c|}
\hline gloss & Nenets & Enets & Nganasan & Selkup & Kamas & Mator & etymology \\
\hline $\begin{array}{l}15 \mathrm{a} . \\
\text { cold }\end{array}$ & $\begin{array}{l}\text { tî̀ti, tî̀ci= } \\
\text { tećd } d a_{\mathrm{RNS}}\end{array}$ & $\begin{array}{l}\text { teti(re) })^{\mathrm{Ch}} \\
\text { tetide }^{\mathrm{B}}\end{array}$ & tasiti & $\begin{array}{l}\text { täšedal }^{\mathrm{N}} \\
\text { tassundi- }_{\text {MO,K }}\end{array}$ & $\begin{array}{l}\text { šišti } \\
\text { šišigäa }\end{array}$ & $\begin{array}{l}\text { tüuchtä̈ }{ }^{\mathrm{Mt}} \\
\text { dékte }^{\mathrm{Tg}}\end{array}$ & 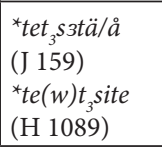 \\
\hline $15 b$. & japada $a_{\mathrm{RNS}}$ & & & & & & $\begin{array}{l}\text { cf. japă(ś) be } \\
\text { cold }\end{array}$ \\
\hline $15 \mathrm{c}$. & yerm $_{\mathrm{RNS}}$ & & & & & & $\begin{array}{l}\text { *ermâ north } \\
\text { (J 22) }\end{array}$ \\
\hline $15 \mathrm{~d}$. & & & & $\begin{array}{l}\text { kâil, kael }^{\mathrm{N}} \\
\text { kâné }^{\mathrm{B}, \text { Tas }}= \\
\text { qantipipil } \\
\text { Tas/_ }\end{array}$ & & & $\begin{array}{l}\text { cf. }{ }^{*} k \partial \hat{n} \text { tä- } \\
\text { frieren } \\
\text { (J 53; H 265) } \\
<\text { U *konta } \\
\text { (R 176-77) } \\
\end{array}$ \\
\hline $15 \mathrm{e}$. & & & & $\begin{array}{l}\text { časiqil } \\
\text { Tas/ }\end{array}$ & & & cf. čāsi sea \\
\hline $\begin{array}{l}\text { 16a. } \\
\text { come }\end{array}$ & $\begin{array}{l}\text { tûryadm } \\
\text { tôdm }\end{array}$ & $\begin{array}{l}\text { toaro }^{\mathrm{Ch}} \\
\text { toado }^{\mathrm{B}}\end{array}$ & tûam & $\begin{array}{l}t_{\bar{a} a k^{\mathrm{N}}} \\
t \hat{u} a \eta^{\mathrm{MO}}\end{array}$ & šolam & $\begin{array}{l}\text { (dèlym-) } \\
\text { doi }{ }^{\mathrm{Kg}} \\
\text { come } \\
\text { (here) }\end{array}$ & $\begin{array}{l}{ }^{*}{ }^{\text {toj- }}{ }^{*}{ }^{*} \text { tuj- } \\
\text { (J 164) } \\
<\mathrm{U}{ }^{*} \text { tule } \\
\text { (R 535) }= \\
{ }^{*} \text { toli- }\left(\mathrm{S}_{1} \text { 540) }\right. \\
\end{array}$ \\
\hline $16 \mathrm{~b}$. & haje $($ ad)m & & & & & & \\
\hline $16 c$. & & & & & kandagam & & 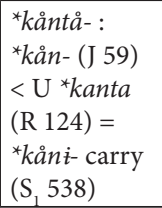 \\
\hline 16d. & & & & & šônagam & & \\
\hline $16 e$. & & & & & molảm $_{\mathrm{D}}$ & & \\
\hline $\begin{array}{l}\text { 17a. } \\
\text { die }\end{array}$ & hâdm & $\begin{array}{l}\text { kâro }{ }^{\text {'Ch }} \\
k_{a ̂} d o^{\text {B }}\end{array}$ & kụ̂am & $\begin{array}{l}\text { kuak }^{\mathrm{N}} \\
\text { kuyan }^{\mathrm{B}, \mathrm{Tas}}\end{array}$ & khüläm & $\begin{array}{l}\text { chaàsiga- } \\
\text { ani }^{\mathrm{Kg}} \\
\text { ki-myndži }^{-} \\
\text {-gulja- } \\
m z^{\mathrm{Mt}}\end{array}$ & $\begin{array}{l}{ }^{*} \text { kåô- } \\
(\mathrm{J} 56-57 ; \\
\mathrm{H} 367)<\mathrm{U} \\
{ }^{*} k o \overline{l a}(\mathrm{R} 173) \\
={ }^{*} k a ̊ x l i- \\
\left(\mathrm{S}_{1} \text { 538) }\right.\end{array}$ \\
\hline $17 \mathrm{~b}$. & & & & üre- $^{\mathrm{Ty}} / \mathrm{DA}$ & örerläm & & $\begin{array}{l}\text { < Tk: Kyzyl } \\
\text { ölär-(Jo 252) }\end{array}$ \\
\hline $\begin{array}{l}18 \mathrm{a} . \\
\text { dog }\end{array}$ & wueno & $\begin{array}{l}\text { bî', } \\
\text { g. buno }{ }^{\mathrm{Ch}} \\
\text { bunneke }\end{array}$ & bây & $\begin{array}{l}\text { kanak }^{\mathrm{B}, \mathrm{Tas}} \\
\text { kännay }^{\mathrm{MP}}\end{array}$ & men & $\begin{array}{l}\text { bön } n^{\mathrm{Mt}, \mathrm{Kg}} \\
\text { bun }^{\mathrm{Tg}}\end{array}$ & $\begin{array}{l}\text { *wen (J 173; } \\
\text { H 117) }\end{array}$ \\
\hline $18 \mathrm{~b}$. & $\begin{array}{l}\text { jandu/o } \\
\text { d. with } \\
\text { short } \\
\text { hair \& } \\
\text { standing } \\
\text { ears }\end{array}$ & & & & & & $\begin{array}{l}\text { cf. Tn }{ }^{*} j a n- \\
\text { daku } \\
\text { racooon dog, } \\
\text { wolverine }\end{array}$ \\
\hline
\end{tabular}


Václav Blažek

\begin{tabular}{|c|c|c|c|c|c|c|c|}
\hline gloss & Nenets & Enets & Nganasan & Selkup & Kamas & Mator & etymology \\
\hline $\begin{array}{l}\text { 19a. } \\
\text { drink }\end{array}$ & jideryâdm & $\begin{array}{l}\text { biribo } \\
\text { bidibo }^{\mathrm{B}}\end{array}$ & bede'am & $\begin{array}{l}\text { ütam }^{\mathrm{B}} \\
\text { üternaฑ }^{\text {Tas }}\end{array}$ & bitläm & & $\begin{array}{l}{ }^{*} \text { witä- } \\
{ }^{*} \text { witôj- : } \\
{ }^{*} \text { wit water } \\
\text { (J 176) }\end{array}$ \\
\hline $19 \mathrm{~b}$. & jâbiedm & & & & & & \\
\hline $19 c$. & $\begin{array}{l}\text { nerpă(ś) } \\
\text { RNS }\end{array}$ & $i h r u \check{s}^{\mathrm{K}} /_{\mathrm{M}}$ & & $\hat{\imath} r a^{\mathrm{Ket}} / \mathrm{D}$ & & $\begin{array}{l}\ddot{o r s u}^{\mathrm{Mt}, \mathrm{Kg}, \mathrm{Tg}} \\
\text { urnjamz }^{\mathrm{Mt}}\end{array}$ & $\begin{array}{l}{ }^{*} \text { er- (J 21-22; } \\
\text { H 249) < } \\
\text { U * } ̈ \text { ur3 (R 85) }\end{array}$ \\
\hline $\begin{array}{l}20 a . \\
\text { dry }\end{array}$ & hâsui & $\begin{array}{l}\text { kasua }^{\mathrm{Ch}} \\
\text { kasue }^{\mathrm{B}}\end{array}$ & kośụa & $\begin{array}{l}\text { (kuuska }{ }^{\mathrm{K}} \\
\text { seicht) }\end{array}$ & \begin{tabular}{|l|}
$(k \hat{o} l a m=$ \\
$k o^{\prime}-$ Do dry \\
up \\
if it be- \\
longs here\})
\end{tabular} & & $\begin{array}{l}{ }^{*} k a ̊ s(\hat{\partial})-v b .(J \\
60-61)< \\
U^{*} k o s ́ k \dot{-} \\
\left(S_{1} 537\right)\end{array}$ \\
\hline $20 \mathrm{~b}$. & tyrabej $_{\mathrm{RNS}}$ & & & $\begin{array}{l}\text { tirawally } \\
\text { DA }\end{array}$ & & $\begin{array}{l}\left(\text { tyry }{ }^{\mathrm{Mt}}\right. \\
\text { hard) }\end{array}$ & 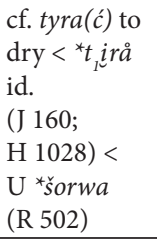 \\
\hline $20 c$. & & & & \begin{tabular}{|l|} 
čégemba- \\
Tas,B \\
tekipipl $l^{\text {Tas }}{ }_{\mathrm{E}}$ \\
\end{tabular} & & & \\
\hline $20 \mathrm{~d}$. & & & & & kumay & & \\
\hline $20 \mathrm{e}$. & & & & & $\frac{\underline{\operatorname{ararbi}}_{\mathrm{D}}}{(+ \text { mager })}$ & & $\begin{array}{l}\text { < Tk: cf. } \\
\text { Chag aruk } \\
\text { mager } \\
\text { (Rä 22) }\end{array}$ \\
\hline $20 f$. & & & & & & ${\underline{\text { kálulex }^{\mathrm{Mt}}}}^{\mathrm{Tg}}$ & $\begin{array}{l}\text { < Chag qaq- } \\
\text { liq dryness or } \\
\text { Ket kuoley } \\
\text { (H 462) }\end{array}$ \\
\hline $\begin{array}{l}21 \mathrm{a} . \\
\text { ear }\end{array}$ & $h \hat{a}$ & $k \hat{u}^{\mathrm{Ch}}, k \hat{o}^{\mathrm{B}}$ & kou & $\begin{array}{l}k \hat{o}^{\mathrm{N}}, k \hat{u}^{\mathrm{B}} \\
=k \bar{o} \\
q o^{\mathrm{Tas}} / \mathrm{E}\end{array}$ & $k u=k^{\prime} \grave{u}_{\mathrm{D}}$ & $\begin{array}{l}g u(k)^{\mathrm{Mt}} \\
k u-k d a^{\mathrm{Mt}}\end{array}$ & $\begin{array}{l}{ }^{*} k a ̊ w(\mathrm{~J} 62 ; \\
\mathrm{H} 569)< \\
\mathrm{U}{ }^{*} k a ̊ w i \\
(J \mathrm{~J} 253)= \\
{ }^{*} \text { kåwi } \\
(\mathrm{S}, 538)\end{array}$ \\
\hline $21 \mathrm{~b}$. & & & & $\begin{array}{l}\text { üyulsan = } \\
\ddot{u} \eta k i l s a- \\
t^{\mathrm{Tas}} / \mathrm{E}\end{array}$ & & & $\begin{array}{l}\text { cf. ünkiti- } \\
\text { listen } \\
\text { üntičici-qo } \\
\text { hear }\end{array}$ \\
\hline $\begin{array}{l}22 \mathrm{a} . \\
\text { earth }\end{array}$ & $j a, j e a$ & $d a^{\mathrm{B}}, j \hat{a}^{\mathrm{Ch}}$ & \begin{tabular}{|l|} 
(jua sand, \\
ashes)
\end{tabular} & $\begin{array}{l}\check{c}^{\mathrm{N}}, \\
t^{\mathrm{K}} u^{\mathrm{K}, \mathrm{MO}}\end{array}$ & $t^{\prime} u=d^{\prime} u_{\mathrm{D}}$ & $\begin{array}{l}\text { dshal } \\
\text { tčja }{ }^{\mathrm{Mt}} \\
\text { dscha }^{\mathrm{Tg}} \\
\end{array}$ & $\begin{array}{l}\text { *jåâ (J 36-37; } \\
\text { H 160) }\end{array}$ \\
\hline $22 b$. & & & $\begin{array}{l}\text { mou } \\
\text { mamaru }\end{array}$ & $\begin{array}{l}\text { (mači } \\
\text { forest, } \\
\text { tundra) }\end{array}$ & $\begin{array}{l}\text { (mija } \\
\text { mountain) }\end{array}$ & $\begin{array}{l}\left(\text { bija } a^{\mathrm{Mt}}\right. \\
\text { moun- } \\
\text { tain) }\end{array}$ & $\begin{array}{l}{ }^{*} m \text { âjå (J 85); } \\
\text { cf. \#55g; } \\
\text { A }_{1} 22-23: \\
\mathrm{U}^{*} m u \delta^{\prime} a\end{array}$ \\
\hline
\end{tabular}


On the classification of the Samoyedic languages

\begin{tabular}{|c|c|c|c|c|c|c|c|}
\hline gloss & Nenets & Enets & Nganasan & Selkup & Kamas & Mator & etymology \\
\hline $\begin{array}{l}23 a . \\
\text { eat }\end{array}$ & yamau & $\begin{array}{l}\text { omabo }^{\mathrm{Ch}} \\
\text { oma }^{\mathrm{B}} / \mathrm{Te}\end{array}$ & yamu'ama & $\begin{array}{l}\text { amnak }^{\mathrm{K}} \\
\text { amarna- } \\
y^{\text {Tas,B }}\end{array}$ & amńam & amlamb $^{\mathrm{Kb}}$ & $\begin{array}{l}\text { *âm- (J 15; } \\
\text { H 807) }\end{array}$ \\
\hline $23 b$. & & & & & 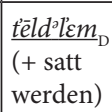 & & $\begin{array}{l}<\text { Sag čeldä } \\
\text { be pregnant }\end{array}$ \\
\hline $23 c$. & & & & & & $\begin{array}{l}\text { hörgu- } \\
m a^{\mathrm{Mt}}\end{array}$ & $\begin{array}{l}{ }^{*} \text { por- (J 127; } \\
\text { H 316)< } \\
\text { U ^pure- } \\
\text { (R 405-06) }\end{array}$ \\
\hline $\begin{array}{l}24 a . \\
\text { egg }\end{array}$ & & mona & mánu & & $\begin{array}{l}\text { münü' }= \\
\text { munoi }_{\mathrm{D}}\end{array}$ & muny $y^{\mathrm{Kb}}$ & $\begin{array}{l}{ }^{*} \text { mânå (J 86) } \\
<\mathrm{U}{ }^{*} \text { muna } \\
(\mathrm{R} 285)= \\
{ }^{*} \text { munå } \\
(\mathrm{S}, 538)\end{array}$ \\
\hline $24 \mathrm{~b}$. & $\begin{array}{l}\text { sârau }= \\
\text { sar'ń }_{\text {RNS }}\end{array}$ & & & & & & \\
\hline $24 c$. & & & & $\begin{array}{l}\text { ńâbi } i^{\mathrm{N}}, \\
n ́ a ̂ p i^{\mathrm{K}}\end{array}$ & & & $\begin{array}{l}=n \bar{a} p-\bar{\imath} \\
\text { duckling } \\
\text { (DA 1630) }\end{array}$ \\
\hline $24 \mathrm{~d}$. & & & & kegai $^{\mathrm{MO}}$ & & & $\begin{array}{l}\text { < Tk: Tatar } \\
k \ddot{u} k a j \\
\text { (DA 2123) } \\
\end{array}$ \\
\hline $24 \mathrm{e}$. & & & & 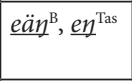 & & & $\begin{array}{l}\text { cf. Ket } \varepsilon ? j, \text { pl. } \\
\bar{e} \eta \text { id. }\end{array}$ \\
\hline $24 f$. & & & & & & $\begin{array}{l}\frac{s c h l u i}{}^{\mathrm{Mt}, \mathrm{Tg}} \\
\underline{\text { schílui }}^{\mathrm{Kg}}\end{array}$ & \begin{tabular}{|l}
$<$ Kott šulei \\
egg \\
$(\mathrm{H} 883)$
\end{tabular} \\
\hline $\begin{array}{l}25 a . \\
\text { eye }\end{array}$ & $\begin{array}{l}\text { saeu, } \\
\text { haem }^{\text {Knd }}\end{array}$ & $s e i$ & śaime & $\begin{array}{l}s a i^{\mathrm{Tas}, \mathrm{B}, \mathrm{K}}, \\
h a i^{\mathrm{N}}, \\
s a i j i^{\mathrm{NP}}\end{array}$ & sima & $\begin{array}{l}\text { schíme }^{\mathrm{Mt}} \\
\text { schí- } \\
\text { medä }\end{array}$ & $\begin{array}{l}{ }^{*} \text { sâjmä }(\mathrm{J} \\
\text { 132; H 886)< } \\
\mathrm{U} \text { *śilmä } \\
\left(\mathrm{R} 479 ; \mathrm{S}_{1}\right. \\
540)\end{array}$ \\
\hline $\begin{array}{l}26 a . \\
\text { fat } n .\end{array}$ & jur & $\begin{array}{l}\frac{j u^{\mathrm{B}}}{}, \\
\text { g. juro' }\end{array}$ & $\begin{array}{l}j i r \\
\underline{d i r}_{\text {NRN }}\end{array}$ & 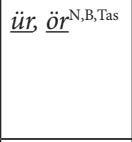 & $\underline{\text { d'uuŕr }}_{\mathrm{D}}$ & $\underline{d z ̌ j u r b}^{\mathrm{Mt}}$ & $\begin{array}{l}{ }^{*} \text { jür (J 50; } \\
\text { H 239) } \\
<\mathrm{Tk}^{*} \bar{u} \dot{r} r \\
(\mathrm{AX} 72)\end{array}$ \\
\hline $26 \mathrm{~b}$. & & $\begin{array}{l}j \hat{u} / j \hat{o}^{\mathrm{Ch}}, \mathrm{g} . \\
j \hat{u s o} / j \hat{u s} o^{\mathrm{B}}\end{array}$ & & čoš́t Tas $/ \mathrm{E}$ & & & ${ }^{*}$ jojs (J 46) \\
\hline $26 c$. & & $\begin{array}{l}\text { (séla } \\
\text { dissolved } \\
\text { fat) }\end{array}$ & & $\begin{array}{l}\text { (siileng } \\
\text { adj.) }\end{array}$ & $s i l=s i \pi_{\mathrm{D}}$ & $s y l b^{\mathrm{Kb}}$ & $\begin{array}{l}{ }^{*} \text { selâ }{ }^{*} \text { sila } \\
(\mathrm{J} 140)< \\
\mathrm{U} \text { *śilä } \\
(\mathrm{R} 478) \\
\end{array}$ \\
\hline $26 \mathrm{~d}$. & $\begin{array}{l}t u t^{\mathrm{Njalina} /} / \mathrm{Le} \\
(+ \text { Talg})\end{array}$ & $\begin{array}{l}\text { (tudute } e^{\mathrm{B}} \\
\text { turute }^{\mathrm{Ch}} \\
\text { adj.) }\end{array}$ & $\begin{array}{l}\check{c} \ddot{u}{ }^{\text {NRN }} \\
\left(t u{ }^{2}\right. \\
\text { Renntier- } \\
\text { talg) }\end{array}$ & & & $\begin{array}{l}t u n^{\mathrm{Mt}, \mathrm{Tg}} \\
t u t t^{\mathrm{Kg}}\end{array}$ & $\begin{array}{l}{ }^{*} t_{1} u_{1} t_{1}(\mathrm{~J} \mathrm{166}) \\
={ }^{*} t_{1} u t_{1} \\
(\mathrm{H} 1106)< \\
\mathrm{U}{ }^{*} \text { s } 8 t_{3} \\
(\mathrm{R} 453)\end{array}$ \\
\hline
\end{tabular}


Václav Blažek

\begin{tabular}{|c|c|c|c|c|c|c|c|}
\hline gloss & Nenets & Enets & Nganasan & Selkup & Kamas & Mator & etymology \\
\hline $\begin{array}{l}27 a . \\
\text { feather }\end{array}$ & $t u$, to & (tua wing) & $\begin{array}{l}\text { túe } \\
(t u \text { Miller } \\
\text { wing }(a) \\
\text { wing }\end{array}$ & $t u^{\mathrm{N}}, t \hat{u}^{\mathrm{B}}$,Tas & & $\begin{array}{l}\text { tu } \\
\text { tógt }\end{array}$ & $\begin{array}{l}{ }^{*} \text { tuâj (J 166) } \\
\text { or }{ }^{*} \text { tua } \\
(\mathrm{H} 1070)< \\
\mathrm{U}^{\star}{ }^{*} \text { tulka } \\
(\mathrm{R} 535)= \\
{ }^{*} \text { tuxli- }\left(\mathrm{S}_{1}\right. \\
540)\end{array}$ \\
\hline $27 \mathrm{~b}$. & & tábu $u^{\text {'Ch }}$ & & & & & (M 209) \\
\hline $27 \mathrm{c}$. & (tar hair) & $\begin{array}{l}\left(\text { tồ }{ }^{\mathrm{C} h}, \mathrm{~g} .\right. \\
\text { tốro' })\end{array}$ & $\frac{\text { tar' }^{\prime}}{(+ \text { hair })}$ & $\underline{t a r}_{\mathrm{Pr}}$ & $\begin{array}{l}\frac{\text { ther }}{(+ \text { hair })=} \\
t^{\prime} \hat{\partial} r_{\mathrm{D}}\end{array}$ & $\begin{array}{l}\underline{(t e r r}^{\mathrm{Tg}} \\
\text { hair) }\end{array}$ & $\begin{array}{l}{ }^{*} \text { târ (J 149: } \\
\text { hair)< } \\
\text { Mo tar, see } \\
\text { \#36b }\end{array}$ \\
\hline $27 \mathrm{~d}$. & & & $\begin{array}{l}\text { d'eftuńén- } \\
s a_{\mathrm{KS}}\end{array}$ & & & & \\
\hline $\begin{array}{l}28 a . \\
\text { fire }\end{array}$ & $t u$ & $t \hat{u}^{\mathrm{Ch}}, t u^{\mathrm{B}}$ & tui & $t \ddot{u}^{\mathrm{N}}, t \ddot{u}^{\mathrm{OO}, \check{\mathrm{C}}}$ & 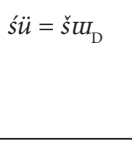 & $t u i^{\mathrm{Mt}}, t u i^{\mathrm{Kg}}$ & $\begin{array}{l}{ }^{*} \text { tuj }(\mathrm{J} \mathrm{166)<} \\
\mathrm{U}^{\star} \text { tule } \\
(\mathrm{R} 535)= \\
{ }^{*} \text { tuli }\left(\mathrm{S}_{1} 540\right)\end{array}$ \\
\hline $\begin{array}{l}29 a . \\
\text { fish }\end{array}$ & hâlea & $\begin{array}{l}\text { karé }^{\mathrm{Ch}}, \\
\text { kare }^{\mathrm{B}}\end{array}$ & kole & $\begin{array}{l}\text { kuel }^{\mathrm{N}, \mathrm{B}} \\
\text { kuele }^{\mathrm{Tas}, \mathrm{NP}}\end{array}$ & kola & $\begin{array}{l}\text { chall }^{\mathrm{Mt}} \\
\text { kale }^{\mathrm{Kg}}\end{array}$ & 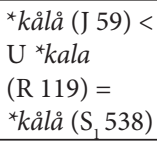 \\
\hline $\begin{array}{l}30 \mathrm{a} . \\
\text { fly v. }\end{array}$ & t/tî̀ryadm & $\begin{array}{l}\text { tî̀yaro' }^{\text {'Ch }} \\
\text { tî̀nedo' }^{\text {B }}\end{array}$ & taérim & $\begin{array}{l}\text { tîmbak } \\
\text { tîmbay- } \\
\text { K,Tas,B } \\
\text { tiľ̌̌erna- } \\
\eta^{\mathrm{B}, \text { Tas }} \\
\end{array}$ & & $\operatorname{dijam}^{\mathrm{Mt}}$ & $\begin{array}{l}{ }^{\star} t i(j)-(\mathrm{J} 161 ; \\
\mathrm{H} 1009 ; \mathrm{DA} \\
965)\end{array}$ \\
\hline $30 \mathrm{~b}$. & & & & $\begin{array}{l}\text { wašejak }^{\mathrm{N}} \\
\text { wašeteya- }^{\text {Tas }} \\
\eta^{\text {Tas }} \\
=\text { wešiqo- } \\
\text { Tas/ } \\
\text { (+ au- } \\
\text { fheben, } \\
\text { aufste- } \\
\text { hen })\end{array}$ & & & \\
\hline $30 \mathrm{c}$. & & & & & nergüläm & & \\
\hline $\begin{array}{l}\text { 31a. } \\
\text { foot }\end{array}$ & yae & $\eta \hat{a}^{\mathrm{Ch}}, \eta \hat{o}^{\mathrm{B}}$ & yoai & & $\begin{array}{l}\ddot{u} j \ddot{u}, u j \ddot{u}= \\
u \dot{i} u, u_{n} u_{\mathrm{D}}\end{array}$ & $\begin{array}{l}\text { pl. } \\
\text { áschta }{ }^{\mathrm{Mt}} \\
\text { cf. hoi }{ }^{\mathrm{Mt}} \\
\text { knee } \\
<^{*} \text { hoy-oj }\end{array}$ & $\begin{array}{l}{ }^{*} \ddot{a}(\hat{\partial}) j(\mathrm{~J} \mathrm{17} \\
\mathrm{H} 9,315)\end{array}$ \\
\hline $31 b$. & $\begin{array}{l}(\text { toba } \\
\text { hRN } \\
\text { hoof })\end{array}$ & & & $\begin{array}{l}\text { tob }^{\mathrm{N}}, \\
\text { tope } e^{\text {Tas }} \\
=\text { topi }^{\text {Tas } /}\end{array}$ & & & ${ }^{*}$ topå (J 166) \\
\hline $31 \mathrm{c}$. & & & & & ${\underline{u} u u^{\prime}}_{\mathrm{D}}$ & & $\begin{array}{l}<\text { Buryat ula } \\
\text { Sohle }\end{array}$ \\
\hline
\end{tabular}


On the classification of the Samoyedic languages

\begin{tabular}{|c|c|c|c|c|c|c|c|}
\hline gloss & Nenets & Enets & Nganasan & Selkup & Kamas & Mator & etymology \\
\hline $\begin{array}{l}32 a . \\
\text { full }\end{array}$ & $\begin{array}{l}\text { pânta, } \\
\text { pâny }\end{array}$ & $\begin{array}{l}\text { faddi'a, } \\
\text { faddite }^{\mathrm{B}}\end{array}$ & & & & & \\
\hline $32 \mathrm{~b}$. & & & $\begin{array}{l}\text { mintu- } \\
\text { tia }= \\
\text { mün- } \\
\text { tütüo } \\
\text { NRN }\end{array}$ & & & & \\
\hline $32 \mathrm{c}$. & & & deragaz- & & & & \\
\hline $32 \mathrm{~d}$. & & & dülsio $_{\mathrm{NRN}}$ & & & & \\
\hline $32 \mathrm{e}$. & & & & $\begin{array}{l}\text { tîr }(m b i- \\
\text { dai }^{\mathrm{NP}} \\
\text { tîrmbedi- } \\
\mathrm{K}, \mathrm{C}, \mathrm{OO} \\
=\text { tiril }^{\text {Tas }}{ }_{\mathrm{E}}\end{array}$ & & & $\begin{array}{l}{ }^{*} \text { tirô } \sim{ }^{*} \text { terô } \\
\text { (J 158) cf. FU } \\
{ }^{*} \text { täw } \delta e \text { full } \\
\text { (R 518; } A_{1} \\
\text { 31) }\end{array}$ \\
\hline $32 \mathrm{f}$. & & & & $\begin{array}{l}\text { sêrbadie }^{\mathrm{K}} \\
\text { sierbadi }^{\mathrm{C}}\end{array}$ & & & \\
\hline $32 \mathrm{~g}$. & & & & & albei & & \\
\hline $32 \mathrm{~h}$. & & & & & & $\begin{array}{l}{ }^{*} \text { mool- } \\
d z ̌ e^{\mathrm{Mt}}\end{array}$ & $\begin{array}{l}\text { (H 687: } \\
\text { ama-mooldže } \\
\text { empty : ama- } \\
\text { non-) }\end{array}$ \\
\hline $\begin{array}{l}\text { 33a. } \\
\text { give }\end{array}$ & mi'idm & $\begin{array}{l}\text { mi'ebo }^{\mathrm{Ch}} \\
m i^{\prime} a^{\mathrm{B}} /_{\mathrm{Gl}}\end{array}$ & mîji'ema & $\begin{array}{l}\text { meap }^{\mathrm{N}} \\
\text { migam }^{\mathrm{oO}} \\
\text { mitam }^{\text {Tas }}\end{array}$ & miläm & mijamz $^{\mathrm{Mt}}$ & $\begin{array}{l}{ }^{*} m i-(\mathrm{J} \mathrm{94;} \\
\mathrm{H} 305) \\
<\mathrm{U}^{*} \text { miy } 3 \\
(\mathrm{R} 275)= \\
{ }^{*} \text { mexi- } \\
(\mathrm{S}, 538)\end{array}$ \\
\hline $33 b$. & $t a s_{\mathrm{RNS}}$ & $\begin{array}{l}\text { terabo }{ }^{\mathrm{Ch}} \\
\text { tedabo }\end{array}$ & tada'ama & $\begin{array}{l}\text { (tăd’a- }{ }^{\mathrm{N}} \\
\text { carry, } \\
\text { bring) }\end{array}$ & $d / t e \theta^{L} l_{\varepsilon}^{\prime} m_{\mathrm{D}}$ & & $\begin{array}{l}{ }^{*} \text { tô- (J 145; } \\
\mathrm{H} 930)< \\
\mathrm{U}{ }^{*} \text { toye } \\
(\mathrm{R} 529): \mathrm{FU} \\
{ }^{*} \text { toxi- bring } \\
\left(\mathrm{S}_{1} 550\right)\end{array}$ \\
\hline $\begin{array}{l}34 a . \\
\text { good }\end{array}$ & $s a(u) w a$ & $\begin{array}{l}\text { sowa, } \\
\text { soera } \\
\text { soed }{ }^{\mathrm{B}}\end{array}$ & & $\begin{array}{l}h w a^{\mathrm{N}}, \\
s u o^{\mathrm{OO}} \\
\operatorname{soma}^{\mathrm{B}, \mathrm{Tas}}\end{array}$ & & & $\begin{array}{l}\text { *sâmå } \\
\text { (J 132); cf. Fi } \\
\text { soma pretty, } \\
\text { cute, sweet < } \\
\text { U *śoma } \\
\text { (AX 127-28) }\end{array}$ \\
\hline $34 \mathrm{~b}$. & & fohadde & & & & & \\
\hline $34 c$. & & & arakara $_{\mathrm{NRN}}$ & & & & \\
\hline $34 \mathrm{~d}$. & & & neimi $i_{\mathrm{NRN}}$ & & & & \\
\hline $34 \mathrm{e}$. & & & 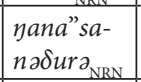 & & & & \\
\hline $34 f$. & & & $\underline{n a \hat{a} g \hat{a}}$ & & & & $\begin{array}{l}<\operatorname{Tn}^{\star} \text { ńaKa } \\
\text { well } \\
(\mathrm{AX} 145)\end{array}$ \\
\hline
\end{tabular}


Václav Blažek

\begin{tabular}{|c|c|c|c|c|c|c|c|}
\hline gloss & Nenets & Enets & Nganasan & Selkup & Kamas & Mator & etymology \\
\hline $34 \mathrm{~g}$. & & & & & 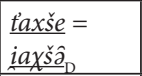 & & $\begin{array}{l}<\text { Tat jaqšy, } \\
\text { jaxšy }\end{array}$ \\
\hline $34 \mathrm{~h}$. & & & & & $\frac{k u \beta a ̊ s}{(+ \text { nice })}$ & & $\begin{array}{l}<\mathrm{Tk} \text { : Brb } \\
\text { kuas char- } \\
\text { ming, Sag kōs } \\
\text { nice (Rä 295; } \\
\text { Jo 214) }\end{array}$ \\
\hline $34 \mathrm{i}$. & & & & & $\begin{array}{l}n e^{\prime} D \partial_{\mathrm{D}} \\
\text { (+ hard, } \\
\text { strong) }\end{array}$ & & \\
\hline $34 \mathrm{j}$. & & & & & & $i n \bar{\imath}^{\mathrm{Mt}}$ & $\begin{array}{l}\text { *enaj (J 23; }_{\text {H 346) }} \\
\text { H }\end{array}$ \\
\hline $\begin{array}{l}35 \mathrm{a} . \\
\text { green }\end{array}$ & jilibeai $^{\text {Knd }}$ & & & & & & $\begin{array}{l}\text { cf. jile(ś) to } \\
\text { live }<{ }^{*}(j) i l a ̈- \\
(\mathrm{J} 27 ; \mathrm{H} \text { 253) } \\
<\mathrm{U}^{*} \text { elä- } \\
(\mathrm{R} 73)\end{array}$ \\
\hline $35 \mathrm{~b}$. & & $\begin{array}{l}\text { poosera- } \\
\text { hach/x } \\
\text { : fóde' gall }\end{array}$ & & $\begin{array}{l}\text { patel }^{\mathrm{B}, \mathrm{Tas}} \\
\text { pačel }^{\mathrm{c}, \mathrm{OOO}}\end{array}$ & & & $\begin{array}{l}\text { cf. }{ }^{*} \text { pâtä gall } \\
\text { (J 115; H 273) } \\
<\mathrm{U}{ }^{*} \text { piša } \\
\text { (R 384); see } \\
\text { also \#100 }\end{array}$ \\
\hline $35 c$. & & & $\begin{array}{l}\text { ńotara- } \\
k u_{\mathrm{NRN}}\end{array}$ & & & & $\begin{array}{l}\text { cf. ńota grass } \\
<{ }^{*} \text { ńåcô< } \\
\mathrm{U}^{*} \text { 'nač } \\
\text { (R 311) }\end{array}$ \\
\hline $35 \mathrm{~d}$. & & & & $\begin{array}{l}\begin{array}{l}\text { noräl } \\
\text { (+blue) }\end{array} \\
\end{array}$ & & & DA 1407 \\
\hline $35 \mathrm{e}$. & & & & & $\underline{\operatorname{seg} i}$ & & $\begin{array}{l}\text { D: yellow; cf. } \\
\text { Soy, Sag sigän } \\
\text { grass }\end{array}$ \\
\hline $35 f$. & & & & & $\begin{array}{l}\frac{k^{\prime} u k^{\prime}}{k^{\prime} \partial k_{D}} \\
(+ \text { blue })\end{array}$ & & < Tat kök id. \\
\hline $35 \mathrm{~g}$. & & & & & & déngöhö ${ }^{\mathrm{Kg}}$ & $\begin{array}{l}{ }^{*} t_{1} \text { âyk } k_{3} \text { blue } \\
(\mathrm{H} 989)\end{array}$ \\
\hline $\begin{array}{l}36 a . \\
\text { hair }\end{array}$ & $\begin{array}{l}\text { üöbta, } \\
\hat{e} a ̈ b t \\
=y e b t_{\mathrm{RNS}}\end{array}$ & ito & yâbta & $\begin{array}{l}o p t^{\mathrm{N}}, \\
o p t e^{\text {Tas }}\end{array}$ & $\begin{array}{l}\ddot{a b d e}= \\
e^{\prime} B D i_{\mathrm{D}}\end{array}$ & $\begin{array}{l}\text { ípte } \\
\text { öbdetä }\end{array}$ & $\begin{array}{l}\text { *eptâ(J 21; } \\
\mathrm{H} 246) \\
<\mathrm{U} * a p t e \\
(\mathrm{R} 14-15)= \\
{ }^{*} \text { ipti }\left(\mathrm{S}_{1} 536\right)\end{array}$ \\
\hline $36 \mathrm{~b}$. & $\underline{t a r}$ & $\begin{array}{l}\frac{\text { to }}{\mathrm{B}}, \underline{\text { tô }} \hat{o}^{\mathrm{Ch}}, \\
\text { g. toŕo' }\end{array}$ & $\frac{\text { tar' }^{\prime}(+ \text { fea- }}{\text { ther })}$ & $\begin{array}{l}\left(\underline{\operatorname{tar}}^{\mathrm{Tas}} / \mathrm{E}\right. \\
\text { +feather, } \\
\text { wool })\end{array}$ & $\frac{t h e r}{(+ \text { feather })} \underline{t}^{\prime} \hat{\partial} r_{\mathrm{D}}$ & $\underline{t e r r}^{\mathrm{Tg}, \mathrm{Mt}}$ & $\begin{array}{l}{ }^{*} \text { târ }(\mathrm{J} 149 ; \mathrm{H} \\
991),<\mathrm{Mo} \text { tar } \\
\text { (tip of) hair } \\
\text { (R } 886 \text { rejects } \\
\text { relation to Ug } \\
{ }^{\star} \text { sä } \gamma(3) r 3 \text { or } \\
\text { Mari šar; cf. } \\
\text { \#27 }\end{array}$ \\
\hline
\end{tabular}


On the classification of the Samoyedic languages

\begin{tabular}{|c|c|c|c|c|c|c|c|}
\hline gloss & Nenets & Enets & Nganasan & Selkup & Kamas & Mator & etymology \\
\hline $36 \mathrm{c}$. & & & & $\begin{array}{l}\text { tuja } \\
\text { tuija } \\
\text { NP }\end{array}$ & & & \\
\hline $36 \mathrm{~d}$. & & & & $k \hat{a} z ̌$ & & & \\
\hline $37 \mathrm{e}$. & & & & neurr ${ }^{\mathrm{T} y} / \mathrm{DA}$ & & & \\
\hline $\begin{array}{l}37 \mathrm{a} . \\
\text { hand }\end{array}$ & yuda & $\begin{array}{l}u r a^{\mathrm{Ch}} \\
u d a^{\mathrm{B}}\end{array}$ & jutu & $u d^{\mathrm{N}}, u t^{\mathrm{B} \text {,Tas }}$ & uda & $\begin{array}{l}u d a-m z^{\mathrm{Mt}} \\
u d a-d a^{\mathrm{Kg}}\end{array}$ & $\begin{array}{l}{ }^{*} u t a ̊(J ~ 30)= \\
{ }^{*} u t a(\mathrm{H} \\
1109)\end{array}$ \\
\hline $\begin{array}{l}38 \mathrm{a} . \\
\text { head }\end{array}$ & yaewa & $\begin{array}{l}\text { abuŕích } \\
e b a^{\mathrm{B}}\end{array}$ & $\begin{array}{l}\text { yaiwua } \\
\text { yаewua }\end{array}$ & & & $\begin{array}{l}a ́ i b a^{\mathrm{Mt}} \\
a i b a-d a^{\mathrm{Kg}}\end{array}$ & $\begin{array}{l}\text { *åjwå (J 17) } \\
=\text { *ajwwa (H } \\
12)< \\
\mathrm{U}^{*} \text { ojwa } \\
(\mathrm{R} 336)= \\
{ }^{*} \text { ojwå }\left(\mathrm{S}_{1}\right. \\
536)\end{array}$ \\
\hline $38 \mathrm{~b}$. & & $\begin{array}{l}\text { (orí }{ }^{{ }^{\mathrm{Ch}}} \text {, } \\
\text { ori }^{\mathrm{B}} \text { top, } \\
\text { height) }\end{array}$ & & $o l^{\mathrm{K}}, u l^{\mathrm{B}}$ & ulu & & $\begin{array}{l}{ }^{*} \text { ola (J 29) } \\
\text { cf. FU *alka } \\
\text { end, } \\
\text { beginning } \\
\text { (R 6-7) }\end{array}$ \\
\hline $\begin{array}{l}39 a . \\
\text { hear }\end{array}$ & $\begin{array}{l}\text { jindile- } \\
\text { adm }\end{array}$ & $\begin{array}{l}\text { udediro' } \\
\text { juseredo' }\end{array}$ & jindi'ema & $\begin{array}{l}\text { ündežap } \\
\text { ünde- } \\
\text { tam }^{\mathrm{B}, \mathrm{Tas}} \\
=\ddot{u} n t i- \\
\text { ćiqo }^{\mathrm{Tas}} / \mathrm{E}_{\mathrm{E}}\end{array}$ & nünüläm & $\begin{array}{l}\text { njumda- } \\
m \tau^{\mathrm{Mt}}\end{array}$ & $\begin{array}{l}\text { *jüntวิ- } \\
(\mathrm{J} 49)= \\
\text { *jünti- } \\
\text { (H 787) }\end{array}$ \\
\hline $39 \mathrm{~b}$. & namdâu & nodado ${ }^{\mathrm{B}}$ & & & & & \\
\hline $\begin{array}{l}40 a . \\
\text { heart }\end{array}$ & seai, siei & $\begin{array}{l}\text { seo }^{\mathrm{Ch}}, \\
\text { seijo }^{\mathrm{B}}\end{array}$ & sa, soa & $\left.s \bar{d} d^{\mathrm{T} y}\right|_{\mathrm{D}}$ & $s \hat{\imath}=s \bar{l}_{\mathrm{D}}$ & 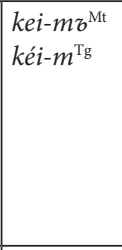 & $\begin{array}{l}{ }^{*} \text { sejâ (J 139; } \\
\mathrm{H} 455)< \\
\mathrm{U} \text { *śü } \delta \ddot{a}\left(-\mathrm{m}_{3}\right) \\
(\mathrm{R} 477): \\
\mathrm{FU} \text { *śüdämi / } \\
{ }^{*} \text { śedmi } \\
(\mathrm{S}, 549)\end{array}$ \\
\hline $40 \mathrm{~b}$. & & $t a \bar{j} j a^{\mathrm{K}} / \mathrm{Pu}$ & & & & & (M 212) \\
\hline $\begin{array}{l}\text { 41a. } \\
\text { horn }\end{array}$ & ńâmd & $\begin{array}{l}\text { eddo } \\
\text { naddo }\end{array}$ & yamta & $\begin{array}{l}\hat{a} m d^{\mathrm{N}}, \\
\hat{a} m d e^{\mathrm{B}, \mathrm{Tas}}\end{array}$ & атпи & $a m d e^{\mathrm{Mt}}$ & $\begin{array}{l}{ }^{*} \ddot{a} m t \hat{a}(\mathrm{~J} 20 ; \\
\mathrm{H} 66)< \\
\mathrm{U}{ }^{*} \text { ayt }{ }^{*}{ }^{*} \text { oyt } 3 \\
(\mathrm{R} 12-13)\end{array}$ \\
\hline $41 \mathrm{~b}$. & & & & & $\underline{m u k u u r}_{D}$ & & $\begin{array}{l}<\text { Tat mügüz, } \\
\text { Chuv } \\
\text { mâiraga }\end{array}$ \\
\hline $42 \mathrm{a} . \mathrm{I}$ & mań & $\operatorname{modi}$ & mannay & $\operatorname{man} / t$ & man & $\operatorname{manz}^{\mathrm{Mt}}$ & $\begin{array}{l}{ }^{*} m \text { ân (J 86; } \\
\text { H 627) } \\
<\mathrm{U}^{*} \text { mun } \\
\text { (R 294) }\end{array}$ \\
\hline
\end{tabular}


Václav Blažek

\begin{tabular}{|c|c|c|c|c|c|c|c|}
\hline gloss & Nenets & Enets & Nganasan & Selkup & Kamas & Mator & etymology \\
\hline $\begin{array}{l}\text { 43a. } \\
\text { kill }\end{array}$ & hâdau & $\begin{array}{l}k_{a r a b o}{ }^{C h} \\
k_{a d a b o}\end{array}$ & $\begin{array}{l}\text { kuada'a- } \\
\text { ma }\end{array}$ & $\begin{array}{l}\text { kuatpam }^{\mathrm{N}} \\
\text { kuetpam- } \\
\text { B,Tas } \\
=\text { qet- } \\
\text { qo } o_{\text {Tas }} \text { E }\end{array}$ & kutlim & kadjamz $^{\mathrm{Mt}}$ & 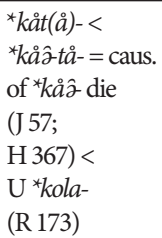 \\
\hline $\begin{array}{l}44 \mathrm{a} . \\
\text { knee }\end{array}$ & pûly, pûle & $\begin{array}{l}\text { fuase } e^{\mathrm{Ch}} \\
\text { fose } \hat{e}^{\mathrm{B}}\end{array}$ & fuagai & $\begin{array}{l}\text { pulsai }^{\mathrm{K}} \\
\text { pûla } \\
\text { saiji }^{\mathrm{NP}} \\
\text { pûle }^{\mathrm{B}, \mathrm{Tas}}\end{array}$ & & $\begin{array}{l}\text { hulu } \\
\text { hulu-dat } \\
\text { (written } \\
\text { in cyrillic } \\
\text { with 2-) }\end{array}$ & $\begin{array}{l}\text { *puâj-+ } \\
\text { *sâjmä eye or } \\
+{ }^{*} \text { le bone } \\
\text { (J 130; } \\
\text { H 323)< } \\
\text { U *polwe } \\
(\mathrm{R} 393)= \\
{ }^{*} \text { po/uxli } \\
\left(\mathrm{S}_{1} 539\right)\end{array}$ \\
\hline $44 \mathrm{~b}$. & & & & & $\begin{array}{l}\sin i= \\
\sin i, \operatorname{sen} i{ }_{D}\end{array}$ & $\begin{array}{l}\text { (schünä } \\
\text { penis; H } \\
920)\end{array}$ & $\begin{array}{l}\text { cf. Ugr *śänčs } \\
\text { id. } \\
\text { (R 471) }\end{array}$ \\
\hline $44 c$. & & & & & & $\begin{array}{l}\text { usjundy } y^{\mathrm{Kg}} \\
\text { (yzýnz }^{\mathrm{Mt}} \\
\text { shoulder) }\end{array}$ & $\begin{array}{l}\text { *esâjn }_{2} \text { limb, } \\
\text { joint (J 22) = } \\
\text { *esoń/n } \\
\text { (H 250); } \\
\text { R } 95 \text { rejects } \\
\text { any connec- } \\
\text { tion with } \\
\text { FU *jäsne id. }\end{array}$ \\
\hline $\begin{array}{l}45 \mathrm{a} . \\
\text { know }\end{array}$ & tênêu & $\begin{array}{l}\text { teńibo }^{\circ \mathrm{Ch}} \\
\text { tenneo }^{\mathrm{B}}\end{array}$ & $\begin{array}{l}\text { tanneje- } \\
\text { ama }\end{array}$ & 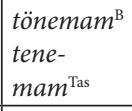 & $\begin{array}{l}\text { (the- } \\
\text { nömäm } \\
\text { think) }\end{array}$ & $\begin{array}{l}\text { tenym-ga- } \\
m \sigma^{\mathrm{Mt}}\end{array}$ & $\begin{array}{l}{ }^{*} \text { tenä(mä)- } \\
\text { (J 157; } \\
\text { H 981) }\end{array}$ \\
\hline $45 \mathrm{~b}$. & & & & & $\begin{array}{l}\text { thümnäm } \\
= \\
t^{`} \text { amnem } m_{\mathrm{D}}\end{array}$ & $\begin{array}{l}\text { (tumdu- } \\
\text { džugura- } \\
m z \\
\text { bemerke) }\end{array}$ & $\begin{array}{l}{ }^{*} t_{1} u m t_{1} \hat{\partial}- \\
(J \text { 167) } \\
\mathrm{U}^{*}{ }^{*} \text { tumte- } \\
(\mathrm{R} 536)= \\
{ }^{*} \text { tumti- } \\
\left(\mathrm{S}_{1} \text { 541) }\right.\end{array}$ \\
\hline $\begin{array}{l}46 a . \\
\text { leaf }\end{array}$ & wuebba & déve & & $\begin{array}{l}\text { čâ } b^{\mathrm{N}}, \\
t a ̂ b^{\mathrm{B}, \text { Tas }}\end{array}$ & $\begin{array}{l}\text { tawa }= \\
t^{\prime} / d a b a_{\mathrm{D}}\end{array}$ & & $\begin{array}{l}\text { ^jäpä (J 41); } \\
\text { cf. Yen *jāpe } \\
\text { id. (St 200; } \\
\text { Jo 116) }\end{array}$ \\
\hline $46 \mathrm{~b}$. & $\begin{array}{l}\text { (yamde } \vec{e}_{\mathrm{Le}} \\
\text { grass) }\end{array}$ & $\begin{array}{l}\text { oddi', g. } \\
-r o^{\prime C h},-d o^{\mathrm{B}}\end{array}$ & $\begin{array}{l}\text { (yamti } \\
\text { willow } \\
\text { leaf) }\end{array}$ & & & & $\begin{array}{l}{ }^{*} \text { amtet }<\mathrm{Tn} \\
{ }^{*} x a b d a-n s a \\
(\mathrm{AX} 100-01)\end{array}$ \\
\hline $46 \mathrm{c}$. & & & $\begin{array}{l}\text { hora- } \\
\delta^{i} a j_{\mathrm{NRN}}\end{array}$ & & & & \\
\hline $46 \mathrm{~d}$. & & & & $\begin{array}{l}\text { (laamb } \\
\text { Fussblatt) }\end{array}$ & & $\begin{array}{l}\text { nambe- } \\
\text { da }^{\mathrm{Mt}} \\
\text { némbja }^{\mathrm{Tg}}\end{array}$ & $\begin{array}{l}\text { *lämpä } ~ \\
\text { *lempä } \\
\text { (J 82; H 736) }\end{array}$ \\
\hline
\end{tabular}


On the classification of the Samoyedic languages

\begin{tabular}{|c|c|c|c|c|c|c|c|}
\hline gloss & Nenets & Enets & Nganasan & Selkup & Kamas & Mator & etymology \\
\hline 47a. lie & wânajû & $\begin{array}{l}\text { banu- } \\
\mathrm{ga}^{\mathrm{B}} / \mathrm{Gl}\end{array}$ & bənüśs $_{\mathrm{NRN}}$ & & & & (cf. M 37) \\
\hline $47 \mathrm{~b}$. & $\begin{array}{l}\text { juseda(ś) } \\
\text { RNS }\end{array}$ & & & & & & \\
\hline $47 c$. & & $\begin{array}{l}\text { barotea- } \\
\text { ro }^{\mathrm{CCh}} \\
\text { badotido }^{\mathrm{B}} \\
\end{array}$ & & & & & \\
\hline $47 \mathrm{~d}$. & & & $\begin{array}{l}\text { kulśâten- } \\
\text { dem }\end{array}$ & & & & \\
\hline $47 \mathrm{e}$. & & & $\begin{array}{l}\text { ngjúndej- } \\
s y_{\mathrm{X}}\end{array}$ & & & & \\
\hline $47 f$. & & & $\begin{array}{l}\text { tońd'ajči- } \\
s_{\mathrm{NRN}}\end{array}$ & & & & \\
\hline $47 \mathrm{~g}$. & & & & \begin{tabular}{|l|} 
eppak $^{\mathrm{N}}$ \\
ippay $^{\mathrm{B}, \mathrm{Tas}}$ \\
\end{tabular} & i’büm & & cf. DA 35 \\
\hline $47 \mathrm{~h}$. & & & & $\begin{array}{l}\dot{a} a c ̌ i- \\
m p i-{ }^{\mathrm{T} y} / \mathrm{DA}\end{array}$ & & & $\begin{array}{l}\text { ćača- put } \\
\text { (DA 1481) }\end{array}$ \\
\hline $47 i$. & & & & & & kyštam $^{\mathrm{Mt}}$ & 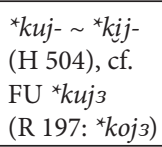 \\
\hline $\begin{array}{l}\text { 48a. } \\
\text { liver }\end{array}$ & $\begin{array}{l}\text { muid }= \\
\text { myd }_{\mathrm{RNS}}\end{array}$ & $\begin{array}{l}\text { muro }{ }^{\mathrm{Ch}} \\
\text { mudo }\end{array}$ & $\begin{array}{l}\text { mita, } \\
\text { g. mida }\end{array}$ & $\begin{array}{l}m y d^{\mathbb{N}} \\
m \hat{i} t e^{\text {Tas }}\end{array}$ & mit $=$ mit $_{\mathrm{D}}$ & & $\begin{array}{l}{ }^{*} \text { mitô }(\mathrm{J} 93)< \\
\mathrm{U}^{*} \text { maksa } \\
(\mathrm{R} 264)= \\
{ }^{*} \text { mikså } \\
(\mathrm{S}, 538)\end{array}$ \\
\hline $48 \mathrm{~b}$. & & & & & & ondarb $^{\mathrm{Mt}}$ & $\begin{array}{l}\text { (H 808: < } \\
\text { IE?); cf. } \\
\text { FU *omte(r3), } \\
\text { e.g. Ma oån- } \\
\text { tèr stomach } \\
\text { (R 338) }\end{array}$ \\
\hline $\begin{array}{l}\text { 49a. } \\
\text { long }\end{array}$ & jâmb & $\begin{array}{l}j a b u^{\mathrm{Ch}} \\
d^{\prime} a ́ b u^{\mathrm{B}} / \mathrm{Te}\end{array}$ & & $\begin{array}{l}\text { čumb }^{\mathrm{N}} \\
\text { t’umbe }^{\mathrm{B} \text {,Tas }}\end{array}$ & $\begin{array}{l}\text { numu }= \\
\text { numo. } \\
{ }_{\mathrm{D}}\end{array}$ & $\begin{array}{l}\text { nanbu }^{\mathrm{Mt}} \\
\left(\text { njámbu }^{\mathrm{Kg}}\right. \\
\text { high })\end{array}$ & 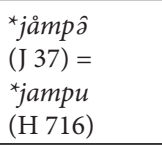 \\
\hline $49 \mathrm{~b}$. & pōndej $j_{\mathrm{RNS}}$ & & & & & & cf. pon' adv. \\
\hline $49 c$. & & & kunta & & & $\begin{array}{l}\text { (kundu- } \\
g u^{\mathrm{Mt}} \\
\text { far) }\end{array}$ & $\begin{array}{l}{ }^{*} \text { kuntâ } \\
\text { (J 77-78; } \\
\text { H 590) }\end{array}$ \\
\hline $49 \mathrm{~d}$. & & & & $\begin{array}{l}\text { sayčc } \partial^{\mathrm{Ket}} / \\
\mathrm{DA}\end{array}$ & & & $\begin{array}{l}\text { *sâycä high } \\
\text { (J 133) }\end{array}$ \\
\hline $49 \mathrm{~d}$. & & & 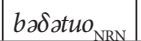 & & & & \\
\hline
\end{tabular}


Václav Blažek

\begin{tabular}{|c|c|c|c|c|c|c|c|}
\hline gloss & Nenets & Enets & Nganasan & Selkup & Kamas & Mator & etymology \\
\hline $\begin{array}{l}50 a . \\
\text { louse }\end{array}$ & $\begin{array}{l}\text { pand'(i')e } \\
\text { panže,-sie }\end{array}$ & & & & & $\begin{array}{l}\text { chÿndz- } \\
s c h i^{\mathrm{Kg}} \\
\text { indž } i^{\mathrm{Mt}}\end{array}$ & $\begin{array}{l}{ }^{*} \text { pans3 } \\
\text { (H 304) }\end{array}$ \\
\hline $50 \mathrm{~b}$. & & $a d d u^{\mathrm{Ch}}$ & yomtun & $\begin{array}{l}\text { un } \check{z}^{\mathrm{N}}, \\
\text { undz } e^{\mathrm{K}} \\
\text { un } \check{z}^{\mathrm{B}, \mathrm{Tas}}\end{array}$ & $\begin{array}{l}\text { ünü }= \\
u: n u_{\mathrm{D}}\end{array}$ & & $\begin{array}{l}\text { *åncâ } ~ \\
{ }^{*} \text { åmcâ (J 18) } \\
<\mathrm{U}{ }^{*} \text { omča } \\
\text { (R 338) }\end{array}$ \\
\hline $\begin{array}{l}\text { 51a. } \\
\text { man }\end{array}$ & hâsawa & $\begin{array}{l}k \hat{a} s a^{\mathrm{Ch}} \\
k \bar{a} s a^{\mathrm{B}} /_{\mathrm{Pu}}\end{array}$ & kиајити & & $\begin{array}{l}-k u z a \\
\left(k^{c} u z a_{\mathrm{D}}\right. \\
\text { Mensch })\end{array}$ & $\begin{array}{l}\text { (kasa, } \\
k a z a^{\mathrm{Mt}} \\
\text { Mensch) }\end{array}$ & $\begin{array}{l}{ }^{*} \text { kåa-så } \\
{ }^{*} \text { Sterblicher } \\
\text { (J 61; H 438) }\end{array}$ \\
\hline $51 b$. & & & $\begin{array}{l}(\text { tybyjkja- } \\
k u \\
\text { boy })\end{array}$ & $\begin{array}{l}t e b^{\mathrm{N}} \\
\text { tippa } \\
\end{array}$ & $\begin{array}{l}\text { thibi= } \\
t^{c} i b i_{\mathrm{D}} \\
(+ \\
\text { husband })\end{array}$ & $\begin{array}{l}{\left(\text { tíhim }^{\mathrm{Mt}}\right.} \\
\text { maritus) } \\
\text { (tigeč }^{\mathrm{Tg}} \\
\text { boy, son } \\
={ }^{*} \text { tihi }+ \\
\text { eči })\end{array}$ & $\begin{array}{l}{ }^{*} \text { tipå (J 163; } \\
\text { H 1014) }\end{array}$ \\
\hline 51c. & & & & \begin{tabular}{|l}
$q u p$, \\
$q^{\mathrm{Tas}} / \mathrm{E}$ \\
$(+\mathrm{Men}-$ \\
sch $)$
\end{tabular} & & & $\begin{array}{l}\mathrm{U}^{\star} k o j(e) m_{3} \\
(\mathrm{R} 168): \\
\mathrm{FU}{ }^{\star} k o j i \\
\text { male } \\
(\mathrm{S}, 543)\end{array}$ \\
\hline $51 \mathrm{~d}$. & & & & & $\begin{array}{l}\text { buu(:)źe } \\
(+ \\
\text { husband) }\end{array}$ & & \\
\hline $\begin{array}{l}52 \mathrm{a} . \\
\text { many }\end{array}$ & yôka & $\begin{array}{l}\hat{o} k a^{\mathrm{Ch}} \\
o k k a^{\mathrm{B}} / \mathrm{Te}\end{array}$ & yôka & $\begin{array}{l}? k o c^{\mathrm{N}} \\
k o t e^{\text {Tas }} \\
(\mathrm{DA} \\
2060)\end{array}$ & $\begin{array}{l}\hat{\imath} g \ddot{o}=i^{\prime} k \partial, \\
i^{\prime} g \hat{\partial}_{\mathrm{D}}\end{array}$ & $u j k o^{\mathrm{Mt}}$ & $\begin{array}{l}{ }^{*} o(\hat{\partial j j}) t 3 k a ̊ \\
(\mathrm{~J} 29)= \\
{ }^{*} \mathrm{ojt}_{3} k a \\
(\mathrm{H} 1117)\end{array}$ \\
\hline $52 \mathrm{~b}$. & & & & $\begin{array}{l}\operatorname{kon}(n)- \\
\partial \eta^{\mathrm{KM}} / \mathrm{DA}\end{array}$ & & & cf. J 75? \\
\hline $52 c$. & & & & $\begin{array}{l}\text { iri }^{\text {Tas }}{ }^{\text {I }} \\
\text { (+ long) }\end{array}$ & & & \\
\hline $\begin{array}{l}53 \mathrm{a} . \\
\text { meat }\end{array}$ & yamsa & $\begin{array}{l}u d a^{\mathrm{Ch}}, \\
o s a^{\mathrm{B}}\end{array}$ & yamsau & $a p s i_{\mathrm{Pr}}$ & & $\begin{array}{l}a m s a^{\mathrm{Mt}, \mathrm{Kg}} \\
a p s a^{\mathrm{Tg}}\end{array}$ & $\begin{array}{l}{ }^{\star} \hat{\partial} m s a ̊ a:{ }^{*} \hat{m}- \\
\text { eat } \\
(\mathrm{J} 15 ; \mathrm{H} 36)\end{array}$ \\
\hline $53 b$. & $\begin{array}{l}\text { (yaija } \\
\text { body; } \\
\text { skin) }\end{array}$ & $\begin{array}{l}\text { (aija body } \\
\text { aja }{ }^{\mathrm{B}} \mathrm{Te}_{\mathrm{Te}}^{+} \\
\text {skin) }\end{array}$ & & $\begin{array}{l}\text { wac }^{\mathfrak{N}^{\mathrm{N}}}, \\
\text { wuet }^{\mathrm{Tas}}\end{array}$ & uja & $\begin{array}{l}\text { (oiuptu }^{\mathrm{Mt}} \\
\text { humidus) }\end{array}$ & $\begin{array}{l}\text { *åjå (J 17; } \\
\mathrm{H} 799)< \\
\mathrm{U}^{*} 0 \delta^{\prime} a \text { raw } \\
\left(\mathrm{A}_{2} 11\right)\end{array}$ \\
\hline $\begin{array}{l}54 \mathrm{a} . \\
\text { moon }\end{array}$ & & & 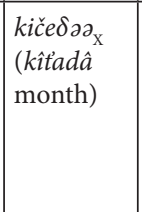 & & $\begin{array}{l}k h i=k i j_{\mathrm{P}} \\
=k^{c} i_{\mathrm{D}}\end{array}$ & kištitz $^{\mathrm{Mt}}$ & $\begin{array}{l}{ }^{*} k i j(\mathrm{~J} 69 ; \\
\mathrm{H} 507)< \\
\mathrm{U}{ }^{*} k u \eta e \\
(\mathrm{R} 211-12) \\
={ }^{*} k i x i \\
(\mathrm{~S}, 537)\end{array}$ \\
\hline $54 b$. & jirî, jiry & $\begin{array}{l}\text { iŕio }^{\mathrm{Ch}} \\
\text { jirie }^{\mathrm{B}}\end{array}$ & & $\begin{array}{l}\ddot{a ̈ r e}^{\mathrm{N}}, \text { ire }^{\mathrm{K}} \\
\text { ireä }^{\mathrm{Tas}}\end{array}$ & & & $\begin{array}{l}{ }^{*}(j) \text { ir3- }(\mathrm{J} \mathrm{28}) \\
\text { cf. }{ }^{*}(j) \text { irs }^{-} \\
\text {grandfather }\end{array}$ \\
\hline
\end{tabular}


On the classification of the Samoyedic languages

\begin{tabular}{|c|c|c|c|c|c|c|c|}
\hline gloss & Nenets & Enets & Nganasan & Selkup & Kamas & Mator & etymology \\
\hline $54 c$. & & & & & urrå $\beta a \bar{a} \beta a_{\mathrm{D}}$ & & $\begin{array}{l}\text { urraba, } \\
\text { uryaba } \\
\text { grandfather; } \\
\text { bear }\end{array}$ \\
\hline $\begin{array}{l}55 \mathrm{a} . \\
\text { moun- } \\
\text { tain }\end{array}$ & $\begin{array}{l}\frac{x o j_{\text {RNS }}}{(=\underline{h o i}} \\
\text { hill })\end{array}$ & $\underline{k u o}_{\mathrm{X}}$ & koaja & 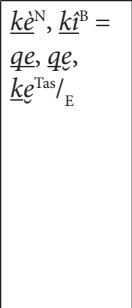 & & & $\begin{array}{l}{ }^{*} k[\text { oa]ja }(\mathrm{AX} \\
105-07 ; \\
\text { X 130)< Ewk } \\
{ }^{*} h o \bar{j} \text { tundra } \\
\text { or Yen }{ }^{*} q a ' j \\
\text { mountain } \\
(\text { St 212); } \\
\text { R 115: } \\
\text { U }{ }^{*} k a \delta ' a\end{array}$ \\
\hline $55 \mathrm{~b}$. & teal & & & & & & \\
\hline $55 \mathrm{c}$. & sea, ša & & & & & & $\begin{array}{l}\text { cf. Ykg šäu } \\
\text { (R 163-64 } \\
\text { separated } \\
\text { it from FU } \\
{ }^{*} \text { kiwi stone) }\end{array}$ \\
\hline $55 \mathrm{~d}$. & & $\log a r^{\mathrm{K}} /_{\mathrm{Pu}}$ & & & & & (M 135) \\
\hline $55 \mathrm{e}$. & & & baru $_{\mathrm{NRN}}$ & $\begin{array}{l}(k \bar{u} r a \\
\text { mountain } \\
\text { ridge })\end{array}$ & $b \bar{o} r_{\mathrm{D}}$ & & $\begin{array}{l}\text { *wårå }< \\
\mathrm{U}^{*} \text { wara } \\
\left(\mathrm{A}_{2} \text { 27) }:\right. \\
\text { FU *wårå } \\
\left(\mathrm{S}_{1} \text { 551) }\right.\end{array}$ \\
\hline $55 f$. & & & $d i k \partial_{\mathrm{NRN}}$ & & & & \\
\hline $55 \mathrm{~g}$. & & & $\begin{array}{l}\text { (mou } \\
\text { earth) }\end{array}$ & $\begin{array}{l}\left(\text { mač }^{\mathrm{Tas}} / \mathrm{E}\right. \\
\text { forest, } \\
\text { tundra })\end{array}$ & $\begin{array}{l}\text { mija }= \\
\text { miia, } \\
\text { maia }_{\mathrm{D}}\end{array}$ & $\begin{array}{l}\text { bija }^{\mathrm{Mt}} \\
\text { biä }\end{array}$ & $\begin{array}{l}{ }^{*} \text { mâjå (J 85; } \\
\text { H 124); cf. } \\
\# 22 b ; \\
A_{1} 22-23: \\
U^{*} m u \delta^{\prime} a \\
\end{array}$ \\
\hline $55 \mathrm{~h}$. & & & & $\frac{\operatorname{ta}}{\mathrm{O}, \mathrm{MO}}^{\mathrm{C}}, \underline{\operatorname{ta\eta }}^{\mathrm{O}-}$ & & & \begin{tabular}{|l|}
$\mathrm{Tk}^{*}$ tay \\
$(\mathrm{DA} 1212)$
\end{tabular} \\
\hline $55 i$. & & & & & 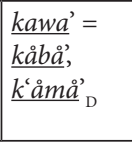 & $\begin{array}{l}\left(k_{a b a k} \mathrm{Kg}\right. \\
\text { eyebrow) }\end{array}$ & $\begin{array}{l}\text { < Tk: Soy } \\
\text { kabak, Bsk } \\
\text { kabak hill } \\
\text { (Jo 155) } \\
\end{array}$ \\
\hline $55 \mathrm{j}$. & & & & & $\underline{k}$ órum $_{\mathrm{D}}$ & & $\begin{array}{l}<\text { MTk } \\
\text { qorum rock } \\
\text { (Jo 196) }\end{array}$ \\
\hline $\begin{array}{l}56 \mathrm{a} . \\
\text { mouth }\end{array}$ & ńa', na $a \eta^{\text {Knd }}$ & $\hat{e}^{\prime C h}, n a^{\prime B}$ & yây & $\hat{a} k^{\mathrm{N}}, \hat{a} \eta^{\mathrm{B}, \mathrm{Tas}}$ & $a y$ & $\begin{array}{l}a g-m a^{\mathrm{Mt}} \\
\text { eng-de }\end{array}$ & $\begin{array}{l}{ }^{* a ̈ y}(\mathrm{~J} \mathrm{20;} \\
\mathrm{H} 76)< \\
\mathrm{U}{ }^{* a \eta j e} \\
(\mathrm{R} 11-12): \\
\mathrm{FU}{ }^{* a ̊ \eta i ~} \\
(\mathrm{~S}, 542)\end{array}$ \\
\hline
\end{tabular}


Václav Blažek

\begin{tabular}{|c|c|c|c|c|c|c|c|}
\hline gloss & Nenets & Enets & Nganasan & Selkup & Kamas & Mator & etymology \\
\hline $\begin{array}{l}\text { 57a. } \\
\text { name }\end{array}$ & $\begin{array}{l}\text { ńim, ńum } \\
\text { ńem }^{\text {Knd }}\end{array}$ & $n^{\prime} \hat{\imath}^{\mathrm{Ch}}, \dot{n} u^{\mathrm{B}}$ & ńim & $\begin{array}{l}\text { ńem } \\
\text { nim }^{\mathrm{B}, \mathrm{OO}}\end{array}$ & nim & $\begin{array}{l}\text { numme- } \\
-d e^{\mathrm{Mt}}\end{array}$ & $\begin{array}{l}{ }^{*} \text { nim }{ }^{*} \text { nüm } \\
(\mathrm{J} \mathrm{102;} \mathrm{H} \mathrm{756)} \\
<\mathrm{U}{ }^{*} \text { nime } \\
(\mathrm{R} \mathrm{305})= \\
{ }^{*} \text { nimi }\left(\mathrm{S}_{1} 538\right)\end{array}$ \\
\hline $\begin{array}{l}\text { 58a. } \\
\text { neck }\end{array}$ & $j \imath \hat{k}, j i k$ & $\begin{array}{l}\text { beko } \delta i^{\mathrm{Ch}} / \mathrm{x} \\
\text { beko }\left.^{\mathrm{B}}\right|_{\mathrm{Pu}}\end{array}$ & $b a k \partial \delta \partial_{\mathrm{M}}$ & $\begin{array}{l}\text { (kuaga }{ }^{\mathrm{K}, \mathrm{NP}} \\
=\text { qeq }^{\mathrm{Tas}} / \mathrm{E} \\
\text { shoulder }) \\
\end{array}$ & $\begin{array}{l}\left(\text { bai' } \hat{\mathrm{d}}_{\mathrm{D}}\right. \\
\text { Nacken })\end{array}$ & $\begin{array}{l}\text { buikö } \\
\text { büik } \ddot{\mathrm{Mt}} d i^{\mathrm{Kg}}\end{array}$ & $\begin{array}{l}\text { *wäjkkâ } \\
\text { (J 173) }\end{array}$ \\
\hline $58 \mathrm{~b}$. & $\begin{array}{l}\text { šo' } \\
(+ \text { Kehle })\end{array}$ & $\begin{array}{l}\left(s \hat{u}, s \hat{o}^{\mathrm{Ch}}\right. \\
\left.s o^{\mathrm{B}} \text { Kehle }\right)\end{array}$ & & $\begin{array}{l}\text { suol }^{\mathrm{B}, \mathrm{Tas}} \\
\text { soi }^{\mathrm{K}}(+ \\
\text { Kehle })\end{array}$ & $\begin{array}{l}\text { soi } \\
\text { (soi Kehle) }\end{array}$ & & $\begin{array}{l}{ }^{*} \text { soj- }{ }^{*} \text { söj- } \\
(\mathrm{J} 142)< \\
\mathrm{U}{ }^{*} \text { śuwe } \\
(\mathrm{R} 492-93)= \\
{ }^{*} \text { śoxi }\left(\mathrm{S}_{1} 540 \text { : }\right. \\
\text { mouth })\end{array}$ \\
\hline $58 \mathrm{c}$. & & & & $\begin{array}{l}a w a i^{\mathrm{MO}, \mathrm{C}} \\
a w o i^{\mathrm{K}}\end{array}$ & & & \\
\hline $58 \mathrm{~d}$. & & & & tāšă $\left.n^{\mathrm{Tas}}\right|_{\mathrm{E}}$ & & & cf. tas back \\
\hline $58 \mathrm{e}$. & & & & $t \varepsilon t i^{\text {Tas }} /_{\mathrm{E}}$ & & & \\
\hline $\begin{array}{l}\text { 59a. } \\
\text { new }\end{array}$ & jedaei & & & & & & \\
\hline $59 \mathrm{~b}$. & & $\begin{array}{l}n a b e^{\mathrm{Ch}} / \mathrm{x} \\
n a b^{\prime} e^{\mathrm{B}} / \mathrm{Te}\end{array}$ & & & & $\begin{array}{l}\text { njáarha }^{\mathrm{Kg}} \\
\text { nara }^{\mathrm{Mt}}\end{array}$ & $\begin{array}{l}\text { *ńarp3 } \\
\text { (H 724; } \\
\text { XE 118) }\end{array}$ \\
\hline $59 c$. & & $\dot{m}^{\prime} e^{\prime} u^{\mathrm{B}} / \mathrm{Te}$ & $\begin{array}{l}\text { mińdjád- } \\
j a_{\mathrm{KS}}= \\
\text { meńda- } \\
d{ }^{\prime}{ }_{\mathrm{NRN}}\end{array}$ & & & & \\
\hline 59d. & & $\begin{array}{l}\text { suami }^{\mathrm{Ch}} / \mathrm{La} \\
(\mathrm{M} \text { 202) }\end{array}$ & & 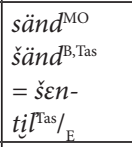 & & & \\
\hline $59 \mathrm{e}$. & & & $\begin{array}{l}b e j- \\
b i " i a_{\mathrm{NRN}}\end{array}$ & & & & \\
\hline $59 f$. & & & & & $\begin{array}{l}\text { the'bde }= \\
t^{\prime} a^{b} D^{\bar{a}}\end{array}$ & & \\
\hline $\begin{array}{l}\text { 60a. } \\
\text { night }\end{array}$ & $p i$ & $f i^{\mathrm{Ch}}, f^{\mathrm{B}}$ & $\begin{array}{l}\text { fì }= \\
h i i_{\mathrm{NRN}} \\
h \text { hinti }_{\mathrm{Te}}\end{array}$ & $p i^{\mathrm{N}, \mathrm{B}}, p e^{\mathrm{M}}$ & $\begin{array}{l}p h i, p h y= \\
p^{\prime} i_{\mathrm{D}}\end{array}$ & $\begin{array}{l}\text { hijndja }{ }^{\mathrm{Mt}} \\
\text { hinde }^{\mathrm{Tg}}\end{array}$ & $\begin{array}{l}{ }^{*} \text { pi (J 123; } \\
\text { H 290) } \\
{ }^{*} \text { pint3 } \\
\text { (H 303); cf. } \\
\text { U *pilm3 } \\
\text { dark } \\
\text { (R 382-83) }\end{array}$ \\
\hline $\begin{array}{l}\text { 6la. } \\
\text { nose }\end{array}$ & $\begin{array}{l}\text { puijea }= \\
\text { pyja }_{\mathrm{RNS}}\end{array}$ & $\begin{array}{l}\text { fuija }^{\mathrm{Ch}} \\
\text { puija }^{\mathrm{B}}\end{array}$ & & $\begin{array}{l}p o \check{c}^{\mathrm{N}} \\
\text { pöt }\end{array}$ & $\begin{array}{l}\text { phîjä }= \\
p^{i} u i e_{\mathrm{D}}\end{array}$ & $h i \ddot{a}^{\mathrm{Mt}}$ & $\begin{array}{l}{ }^{*} p i j a ̊ a{ }^{*} p u j a ̊ \\
\text { (J 122; } \\
\text { H 297) }\end{array}$ \\
\hline $61 \mathrm{~b}$. & & & $\begin{array}{l}\text { yunka = } \\
\text { nüyk }{ }_{\text {NRN }}\end{array}$ & & & & \\
\hline
\end{tabular}


On the classification of the Samoyedic languages

\begin{tabular}{|c|c|c|c|c|c|c|c|}
\hline gloss & Nenets & Enets & Nganasan & Selkup & Kamas & Mator & etymology \\
\hline 61c. & & & & $\begin{array}{l}y n \check{z} l^{\text {B,Tas }} \\
= \\
\text { intäll }^{\text {Tas } / \mathrm{E}}\end{array}$ & & & \\
\hline 61d. & & & & $u k i^{\mathrm{Tas}} / \mathrm{E}$ & & & $\begin{array}{l}{ }^{*} u k \hat{a}(\mathrm{~J} 30 ; \\
\mathrm{H} 1113)\end{array}$ \\
\hline $\begin{array}{l}62 \mathrm{a} . \\
\text { not }\end{array}$ & niś & $\begin{array}{l}\text { isi } \\
\text { ńise }\end{array}$ & $n e^{\prime}$ & $i k a^{\text {Tas }} / \mathrm{E}$ & $\begin{array}{l}e m=\varepsilon m_{\mathrm{D}} \\
\text { ich nicht } \\
\bar{e}, e i_{\mathrm{D}} \text { no }(\mathrm{t})\end{array}$ & igam & 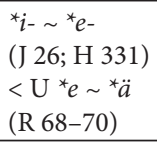 \\
\hline $62 \mathrm{~b}$. & & $\begin{array}{l}\check{s}^{\mathrm{K}}{ }^{\mathrm{K}} / \mathrm{Pu} \text { nicht } \\
\text { (cf. M 205) }\end{array}$ & & 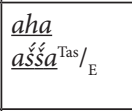 & & & $\begin{array}{l}\text { Tn: } \\
\text { Ewk āčča } \\
\text { no (AX 153) }\end{array}$ \\
\hline $62 c$. & & & & & $\begin{array}{l}n \bar{a}(\gamma \dot{a}) \text { is } \\
\text { not }\end{array}$ & & 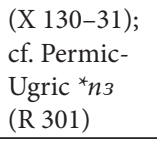 \\
\hline $\begin{array}{l}63 a . \\
\text { one }\end{array}$ & yobpoi & $\begin{array}{l}y \hat{o}^{\mathrm{Ch}}, \\
y \bar{o}^{-\mathrm{B}} / \mathrm{Pr}\end{array}$ & yo'ai' & ?okkir & $o^{\prime} b$ & $o p^{\mathrm{Mt}, \mathrm{Kg}, \mathrm{Tg}}$ & $\begin{array}{l}{ }^{*} o(\hat{\partial}) p-(\mathrm{J} 28 ; \\
\mathrm{H} 794)\end{array}$ \\
\hline $63 \mathrm{~b}$. & & & & & & $e^{e} i l e k^{\mathrm{Mt}}$ & ${ }^{*} a j-($ H 798$)$ \\
\hline $\begin{array}{l}\text { 64a. } \\
\text { person }\end{array}$ & nenete & $\begin{array}{l}\text { ennete }^{3 \mathrm{Ch}} \\
\text { enete }^{\mathrm{B}} /{ }_{\mathrm{Pr}}\end{array}$ & yanasay & & & & $\begin{array}{l}\text { R 627-28 } \\
\text { rejects any } \\
\text { connection } \\
\text { with FMd } \\
\text { *inše; NSm } \text { „man“ is } \\
\text { derived from } \\
\text { *enä real } \\
\text { (J 23; H 96). }\end{array}$ \\
\hline $64 \mathrm{~b}$. & $\begin{array}{l}\ddot{u} b / \\
\text { weri }^{\text {Knd }} \\
x i b j a(\text { ri })_{\mathrm{RNS}} \\
\end{array}$ & & & & & & $\begin{array}{l}\text { cf. xibja who, } \\
\text { xibjari }_{\mathrm{NRS}} \\
\text { whoever }\end{array}$ \\
\hline $64 c$. & & & & $\begin{array}{l}k_{u m}^{\mathrm{MO}, \mathrm{OO}, \mathrm{K}} \\
\text { kume }^{\mathrm{NP}} \\
\text { kop }^{\mathrm{N}} \\
\end{array}$ & & & $\begin{array}{l}\mathrm{U}{ }^{*} k o j(e) m 3 \\
(\mathrm{R} 168)\end{array}$ \\
\hline $64 d$. & & & & & $\begin{array}{l}k u z a= \\
k^{c} u z a_{\mathrm{D}}\end{array}$ & $k a s a^{\mathrm{Mt}}$ & $\begin{array}{l}{ }^{*} \text { kåâ-så } \\
\text { (J 61; H 438) } \\
=\# 51 \mathrm{a} ; \mathrm{cf} . \\
17 \mathrm{a}\end{array}$ \\
\hline $\begin{array}{l}65 \mathrm{a} . \\
\text { rain } \mathrm{n} .\end{array}$ & sâřru/o & $\begin{array}{l}\text { sare }^{\mathrm{Ch}} \\
\text { sare }^{\mathrm{B}}\end{array}$ & soruay & $\begin{array}{l}\text { säro }^{\mathrm{K}}, \\
\text { soro }^{\mathrm{MO}, \check{C}}\end{array}$ & $\begin{array}{l}\text { surnu }= \\
\text { surno }_{\mathrm{D}}\end{array}$ & $s i r r u^{\mathrm{Mt}}$ & $\begin{array}{l}\text { *sårå-jâ(-j): } \\
\text { *sårå- vb. } \\
\text { (J 135-36; } \\
\text { H 908: }{ }^{*} \text { sarö) } \\
\text { < U *śådå- } \\
\left(\mathrm{S}_{1} 540\right)\end{array}$ \\
\hline
\end{tabular}


Václav Blažek

\begin{tabular}{|c|c|c|c|c|c|c|c|}
\hline gloss & Nenets & Enets & Nganasan & Selkup & Kamas & Mator & etymology \\
\hline $\begin{array}{l}66 a . \\
\text { red }\end{array}$ & ńâra & $\begin{array}{l}\text { naggorad- } \\
\text { de } \\
\text { naredad- } \\
d e^{\mathrm{B}} \\
\end{array}$ & & $\begin{array}{l}\text { ńârg } \\
\text { ńärg } \\
\mathrm{B}\end{array}$ & & $\begin{array}{l}\text { narmyn- } \\
d y^{\mathrm{Mt}}\end{array}$ & $\begin{array}{l}\text { *närr-(kâ) } \\
\text { (J 107) } \\
\text { *närma/a- } \\
\text { (H 744) }\end{array}$ \\
\hline $66 \mathrm{~b}$. & & & 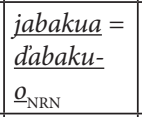 & & & & $\begin{array}{l}<\operatorname{Tn}^{*} \text { dewuk- } \\
\text { ochre } \\
(\text { AX 144) }\end{array}$ \\
\hline $66 c$. & & & & & $\begin{array}{l}k h \bar{u} m \ddot{u}= \\
k^{c} e \underline{m} m u_{\mathrm{D}}\end{array}$ & & $\begin{array}{l}\text { cf. 9a: }{ }^{\star} k e(\hat{\partial}) \\
m \text { blood } \\
(\mathrm{J} 65)\end{array}$ \\
\hline $\begin{array}{l}67 a . \\
\text { road }\end{array}$ & $\eta u^{\prime}$ & $\begin{array}{l}u r i^{\mathrm{Ch}}, u^{\mathrm{B}}, \\
\text { g. } u d o^{\prime}\end{array}$ & $\begin{array}{l}\text { yoajâ }= \\
\text { yuta }\end{array}$ & $\begin{array}{l}\text { watt }(e)^{\mathrm{N}} \\
\text { wuette }\end{array}$ & & & $\begin{array}{l}{ }^{*} u \partial \hat{t} \text { (J 30) } \\
<\mathrm{U}{ }^{*} u t k a \\
(\mathrm{R} 546) \text { or } \\
{ }^{*} u k e t(t 3) \\
(\mathrm{S} 27)= \\
{ }^{*} u k t \dot{t}(\mathrm{~S}, 536)\end{array}$ \\
\hline $67 \mathrm{~b}$. & $\begin{array}{l}\text { (neda } \\
\text { Winter- } \\
\text { weg) } \\
\end{array}$ & & & & $\begin{array}{l}\hat{a} d^{\prime} e= \\
a^{\prime} d^{\prime} e, a^{\prime} t^{\prime} \partial_{\mathrm{D}}\end{array}$ & $\begin{array}{l}i d e^{\mathrm{Mt}} \\
\ddot{o} d e^{\mathrm{Tg}}\end{array}$ & $\begin{array}{l}\text { *etå (J 24; } \\
\text { H 247) }\end{array}$ \\
\hline $67 c$. & $\begin{array}{l}\text { sehaery }_{\mathrm{RNS}} \\
\left(: \text { śehe }_{\mathrm{Te}}\right. \\
\text { hard } \\
\text { snow })\end{array}$ & $\begin{array}{l}\text { seha } a^{\mathrm{Ch}} / \mathrm{X} \\
\text { sehare }^{\mathrm{B} /} / \mathrm{Te}\end{array}$ & & & & & $\begin{array}{l}\text { *sekårâjâ } \\
(\text { S 37-38)< } \\
\text { U *ćäke }(-r 3) \\
\text { (R 31) }\end{array}$ \\
\hline $67 \mathrm{~d}$. & $\begin{array}{l}\text { (sărpä } \\
\text { path) }\end{array}$ & & $\begin{array}{l}\text { saraa/ } \\
\text { sad'a } \\
\text { saruo }_{\mathrm{NRN}} \\
\end{array}$ & $\begin{array}{l}\left(\operatorname{sarpi}^{\mathrm{Tas}} / \mathrm{E}\right. \\
\text { path })\end{array}$ & & & *sârpä (J 134) \\
\hline $67 \mathrm{e}$. & & & & & & $\begin{array}{l}\text { "tanhóo- } \\
\underline{t o}^{\mathrm{Kg}}\end{array}$ & $\begin{array}{l}\text { cf. Ewen hōt } \\
\text { id. < } \\
{ }^{*} \text { pokto } \\
\text { (H 957) }\end{array}$ \\
\hline $\begin{array}{l}68 \mathrm{a} . \\
\text { root }\end{array}$ & wâna/u & $\begin{array}{l}\text { badd } u^{\mathrm{Ch}} \\
\text { badu }{ }^{\mathrm{B}} / \mathrm{Te}\end{array}$ & bântu & 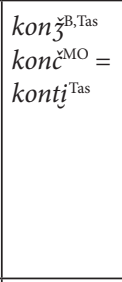 & $\begin{array}{l}\text { muna }= \\
\text { monå }_{\mathrm{D}}\end{array}$ & $\begin{array}{l}\text { mondó } \\
\mathrm{g}, \mathrm{Mt}\end{array}$ & $\begin{array}{l}\text { *wånc3- } \\
\text { *wåncâj } \\
(\mathrm{J} 171 ; \\
\text { H 689)< } \\
\mathrm{U}^{*} \text { wančs } \\
(\mathrm{R} 548-49)= \\
{ }^{*} \text { wåncå } \\
(\mathrm{S}, 541)\end{array}$ \\
\hline $68 \mathrm{~b}$. & & & tóxi $i_{\mathrm{KS}}$ & & & & (X 130) \\
\hline $68 \mathrm{c}$. & & & & $c^{\prime} a \bar{j} \underline{\underline{\partial}}^{\mathrm{Ty}} / \mathrm{DA}$ & & & \\
\hline $68 \mathrm{~d}$. & & & & kor ${ }^{\mathrm{KE}} / \mathrm{DA}$ & & & $\begin{array}{l}\text { : kora deep < } \\
\text { *jorä } \\
(\mathrm{J} 47)\end{array}$ \\
\hline $68 \mathrm{e}$. & & & & & & $\frac{\text { ť́elega- }}{\underline{d e}^{\mathrm{Mt}}}$ & $\begin{array}{l}\text { < Tk: Khak } \\
\text { ćilege, } \mathrm{Kb} \\
\text { jelägi (H 199) }\end{array}$ \\
\hline
\end{tabular}


On the classification of the Samoyedic languages

\begin{tabular}{|c|c|c|c|c|c|c|c|}
\hline gloss & Nenets & Enets & Nganasan & Selkup & Kamas & Mator & etymology \\
\hline $\begin{array}{l}69 a . \\
\text { round }\end{array}$ & śureńa & & & & & & \\
\hline $69 \mathrm{~b}$. & & & $\begin{array}{l}\text { mandaj- } \\
k u_{\mathrm{NRN}}\end{array}$ & & & & \\
\hline 69c. & & & & $\begin{array}{l}\text { pürui }^{\mathrm{NP}} \\
\text { pörui }^{\mathrm{OO}}\end{array}$ & & & $\begin{array}{l}<\mathrm{U}^{*} \text { pirä/e } \\
\text { (R 384) }\end{array}$ \\
\hline $69 \mathrm{~d}$. & & & & $\begin{array}{l}\text { pungola- } \\
\text { Tas/ }\end{array}$ & & & \\
\hline 69e. & & & & & $\frac{\text { thuluka }}{{\underline{t^{\prime} u \pi u k^{\prime}}}_{\mathrm{D}}}=$ & & \begin{tabular}{|l|}
$<$ Yak tula \\
Umkreis (Jo \\
$328-29)$
\end{tabular} \\
\hline $69 f$. & & & & & torta & & $\begin{array}{l}\text { cf. Kalm } \\
\text { doldū id. } \\
\text { (Jo 328-29) }\end{array}$ \\
\hline 69g. & & & & & dạraygo $_{\mathrm{D}}$ & & $\begin{array}{l}\text { Jo } 115 \text { derived } \\
\text { it from Mo } \\
\text { dügürey full, } \\
\text { but semantics } \\
\text { does not cor- } \\
\text { respond }\end{array}$ \\
\hline $69 \mathrm{~h}$. & & & & & & teng $^{\mathrm{Mt}}$ & $\begin{array}{l}\text { < Tk: Kzk } \\
\text { tey Ballen } \\
\text { (H 988) }\end{array}$ \\
\hline $69 \mathrm{i}$. & & & & & & tocorèn $^{\mathrm{Kg}}$ & $\begin{array}{l}<\text { Buryat } \\
\text { tüxerēn id. } \\
\text { (H 1050) } \\
\end{array}$ \\
\hline $\begin{array}{l}70 a . \\
\text { sand }\end{array}$ & & & $\begin{array}{l}\text { düo } o_{\mathrm{NRN}}= \\
\text { jua } \\
(+ \text { ashes })\end{array}$ & & & & 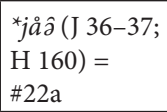 \\
\hline 70aa. & $\begin{array}{l}\text { (jara } \\
\text { sandy) }\end{array}$ & $\begin{array}{l}\text { jâra }{ }^{\mathrm{Ch}} \\
\text { (+ ashes) }\end{array}$ & $\begin{array}{l}\text { (juoru } \\
\text { ochre) }\end{array}$ & $\begin{array}{l}\text { türre } e^{\text {Tas,Kar }} \\
\text { curit }^{\text {Tas }} / \mathrm{E}\end{array}$ & $\begin{array}{l}\text { (durn }{ }_{\mathrm{D}} \\
\text { gravel) }\end{array}$ & $\begin{array}{l}\left(\text { džjara }{ }^{\mathrm{Mt}}\right. \\
\text { arm of } \\
\text { river })\end{array}$ & $\begin{array}{l}\text { *jåârå (J 37; } \\
\text { H 183) }\end{array}$ \\
\hline $70 \mathrm{~b}$. & $t a b$ & $\begin{array}{l}\text { tobo } \check{o}^{\mathrm{B}} / \mathrm{Te} \\
\text { top }^{\mathrm{K}} /{ }_{\mathrm{Pu}} \\
\left(\text { tóbo }^{\mathrm{B}}\right. \\
\text { clay })\end{array}$ & & & & \begin{tabular}{|l}
$\left(t \bar{a}^{\mathrm{Mt}}\right.$ \\
ashes $)$ \\
$\left(\right.$ taha ${ }^{\mathrm{Kg}}$ \\
dirt $)$
\end{tabular} & $\begin{array}{l}{ }^{*} t_{1} \hat{\partial} p \hat{a} \text { (J 148; } \\
\mathrm{H} 937) ;= \\
\# 2 \mathrm{~d}\end{array}$ \\
\hline $70 c$. & & & & $k \bar{o} r a^{\mathrm{Tas}} /_{\mathrm{E}}$ & & & cf. $\# 2$ \\
\hline $70 \mathrm{~d}$. & & & & & $\begin{array}{l}\text { phürä }= \\
p^{\prime} u r \varepsilon_{\mathrm{D}}\end{array}$ & húra $^{\mathrm{Mt}, \mathrm{Tg}}$ & $\begin{array}{l}{ }^{*} \text { puara } \\
\text { (H 330) }\end{array}$ \\
\hline $\begin{array}{l}71 a . \\
\text { say }\end{array}$ & wadieu & $\begin{array}{l}\left(b a \delta e^{\mathrm{B} /}\right. \\
b a \hat{b} o^{\mathrm{Ch}} \\
\text { speak, tell) }\end{array}$ & $\begin{array}{l}\text { buatum, } \\
\text { prt. bua- } \\
\text { juam }\end{array}$ & & & & $\begin{array}{l}\text { *wåô- } \\
(\mathrm{J} 170-71)\end{array}$ \\
\hline $71 \mathrm{~b}$. & heatau & ketus $^{\mathrm{K}} /_{\mathrm{M}}$ & & $\begin{array}{l}\operatorname{ketam}^{\mathrm{B}, \mathrm{Tas}} \\
= \\
\text { keti-qo }^{\mathrm{Tas} / \mathrm{E}}\end{array}$ & & & ${ }^{*} \operatorname{ket}(\hat{\partial})-(\mathrm{J} 66)$ \\
\hline $71 c$. & $\begin{array}{l}\text { mâdm } \\
\text { manźz } \\
\mathrm{RNS}\end{array}$ & $\begin{array}{l}\operatorname{mad}^{\mathrm{B}} / \mathrm{Te} \\
\operatorname{man}^{\prime} \\
\operatorname{mon}^{\mathrm{Ch}} / \mathrm{L}\end{array}$ & $\begin{array}{l}(\text { ku-munsa } \\
\text { what to } \\
\text { say) }\end{array}$ & $\begin{array}{l}(m o m- \\
p a^{\text {Tas }} / \mathrm{E} \\
\text { man sagt })\end{array}$ & mal'cm $_{\mathrm{D}}$ & & $\begin{array}{l}{ }^{*} \text { må(n)- (J 88) } \\
<\mathrm{U}^{*} \text { mon } 3 \\
(\mathrm{R} 290)\end{array}$ \\
\hline
\end{tabular}


Václav Blažek

\begin{tabular}{|c|c|c|c|c|c|c|c|}
\hline gloss & Nenets & Enets & Nganasan & Selkup & Kamas & Mator & etymology \\
\hline $71 \mathrm{~d}$. & & $\begin{array}{l}\text { eitabo }^{\mathrm{Ch}} \\
\text { naitabo }^{\mathrm{B}}\end{array}$ & & & & & \\
\hline 71e. & & & $\begin{array}{l}\text { yamita'a- } \\
\text { ma }\end{array}$ & & & & \\
\hline $71 f$. & & & & täray $^{\mathrm{MO}, \mathrm{K}}$ & & & \\
\hline $71 \mathrm{~g}$. & & & & $\begin{array}{l}\text { tom-qo- } \\
\text { Tas/E }\end{array}$ & & & \\
\hline $71 \mathrm{~h}$. & & & & & $\begin{array}{l}\text { nörbeläm } \\
= \\
\text { nērbəl'sm }\end{array}$ & & \\
\hline $\begin{array}{l}\text { 72a. } \\
\text { see }\end{array}$ & $\begin{array}{l}\text { yodaryau } \\
\text { cf. yadi } \\
\text { visible }\end{array}$ & $\begin{array}{l}\text { (ori }{ }^{\mathrm{Ch}} \\
\text { odi }^{\mathrm{B}} \\
\text { be visible) }\end{array}$ & yadiema & $\begin{array}{l}a t \underline{\underline{\alpha}^{-\mathrm{Ty}} /} \\
\left(a t u-{ }^{\mathrm{Ty}} /\right. \\
=a d a k^{\mathrm{N}} \\
a t a \eta^{\mathrm{B}}, \mathrm{Tas} \\
\text { be visible }) \\
\end{array}$ & & $\begin{array}{l}\text { (adymga }^{\mathrm{Mt}} \\
\text { it seems) }\end{array}$ & $\begin{array}{l}{ }^{*} \hat{t} t \hat{-}-(\mathrm{J} 16 ; \\
\mathrm{H} 7,8)< \\
\mathrm{U}{ }^{*} \text { att3 or } \\
{ }^{*} \text { ott } 3 \\
(\mathrm{R} 20)\end{array}$ \\
\hline $72 b$. & mana'au & 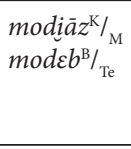 & & \begin{tabular}{|l|} 
mannam- \\
bap $^{\mathrm{N}}$ \\
mannem- $^{\text {Tann }}$ \\
bam $^{\text {Tas }}$ \\
\end{tabular} & $\begin{array}{l}\text { mandôlam } \\
= \\
\text { mån- } \\
\text { dolom }_{\mathrm{D}}\end{array}$ & & $\begin{array}{l}\text { *mânc3- }{ }^{*} \text { (J 86); } \\
\text { cf. Tn }{ }^{*} \text { munsi- } \\
\text { id. (AX 98) }\end{array}$ \\
\hline $72 \mathrm{c}$. & $\begin{array}{l}\text { sîryâdm } \\
\text { xîyyīr }_{\mathrm{Le}}\end{array}$ & $\begin{array}{l}\text { sôyaro }^{\mathrm{Ch}} \\
\text { soyinado }^{\mathrm{B}}\end{array}$ & sayürśa $a_{\mathrm{M}}$ & $\begin{array}{l}\left(\text { sayaqo }_{\mathrm{Pr}}\right. \\
\text { try })\end{array}$ & & $\begin{array}{l}\text { sché- } \\
\text { nemende- } \\
m^{\mathrm{Mt}}\end{array}$ & $\begin{array}{l}\text { *sâyâ- (J 133; } \\
\text { H 875) }\end{array}$ \\
\hline $72 \mathrm{~d}$. & (hou find) & $\begin{array}{l}\left(k o a b o^{C h}\right. \\
k u a b o^{\mathrm{B}} \\
\text { find })\end{array}$ & & $\begin{array}{l}q o-q o^{\mathrm{Tas}} / \mathrm{E} \\
= \\
\text { kogam }^{\mathrm{Tas}}\end{array}$ & kulom $_{\mathrm{D}}$ & $\begin{array}{l}\text { (kojams }^{\mathrm{Mt}} \\
\text { I find) }\end{array}$ & $\begin{array}{l}{ }^{*} k o-\text { to find } \\
\text { (J 72; H 513) } \\
<\mathrm{U}{ }^{*} k o k e- \\
(\mathrm{R} \mathrm{171)}\end{array}$ \\
\hline 72de. & $\begin{array}{l}\left(k \bar{o} n d^{\prime} \varepsilon m_{\mathrm{Le}}\right. \\
\text { try) }\end{array}$ & $\begin{array}{l}k_{0} d d a b o^{\mathrm{Ch}} \\
k u d d a b o^{\mathrm{B}}\end{array}$ & & \begin{tabular}{|l} 
konder- \\
nam $^{\text {Tas }}$ \\
kondör- \\
nam $^{\text {Č }}$ \\
\end{tabular} & & $\begin{array}{l}\text { kondo- } \\
\text { rgomz }^{\mathrm{Mt}}\end{array}$ & $\begin{array}{l}{ }^{*} \text { kont }_{1} \hat{\partial}^{-}: \\
{ }^{*} k_{0}- \\
\text { (J 72; H 546) }\end{array}$ \\
\hline $72 \mathrm{f}$. & & & & $\begin{array}{l}\text { sulal- } \\
\text { č }{ }^{-}{ }^{\mathrm{KU}} / \mathrm{DA}\end{array}$ & & & \\
\hline $\begin{array}{l}73 a . \\
\text { seed }\end{array}$ & sibjako $_{\mathrm{NRS}}$ & & & & & & cf. sibjă dust \\
\hline $73 b$. & & & & $\begin{array}{l}\text { mum- } \\
\text { mol }^{\mathrm{Tu}} /{ }_{\mathrm{DA}}\end{array}$ & & & \\
\hline $73 c$. & & & & 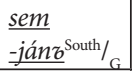 & & & $\begin{array}{l}<\text { Ru semja } \\
\text { (G 177) }\end{array}$ \\
\hline 74a. sit & $\eta \hat{a ̂ m d \hat{y} d m}$ & $\begin{array}{l}\text { adduaro }^{\text {'Ch }} \\
\text { adido }^{\mathrm{B}}\end{array}$ & $\begin{array}{l}\text { yom- } \\
\text { tutum }\end{array}$ & $\begin{array}{l}\hat{a} m d a \eta^{\mathrm{B},-} \\
\mathrm{K}, \mathrm{MO} \\
\hat{a} m t a \eta^{\mathrm{Tas}} \\
= \\
\bar{a} m t i-q o- \\
{ }_{\mathrm{Tas}} / \mathrm{E}\end{array}$ & amnam & $\begin{array}{l}\text { ondoj }^{\mathrm{Mt}} \\
\text { ándai }{ }^{\mathrm{Tg}}\end{array}$ & $\begin{array}{l}\text { *åmtô- } \\
\text { *ämt3- } \\
(\mathrm{J} 17-18 ; \\
\text { H 31)< } \\
\text { U *am3- } \\
\text { (R 8) }\end{array}$ \\
\hline $74 \mathrm{~b}$. & & & & luogan & & & \\
\hline
\end{tabular}


On the classification of the Samoyedic languages

\begin{tabular}{|c|c|c|c|c|c|c|c|}
\hline gloss & Nenets & Enets & Nganasan & Selkup & Kamas & Mator & etymology \\
\hline $\begin{array}{l}75 a . \\
\text { skin }\end{array}$ & $\begin{array}{l}\text { hôba } \\
\text { (+ bark) }\end{array}$ & $\begin{array}{l}k o b a^{\mathrm{B} / \mathrm{Te}} \\
k \hat{o} b \dot{a}^{\mathrm{Ch} / \mathrm{x}}\end{array}$ & $\begin{array}{l}\text { kufu,g. } \\
\text { kubu }\end{array}$ & $\begin{array}{l}k o b^{\mathrm{N}}, \\
k \hat{o} b a^{\check{C}} \\
k o p^{\mathrm{B}, \mathrm{Tas}}\end{array}$ & $\begin{array}{l}\text { kuba, } \\
\text { kuwa }\end{array}$ & $\begin{array}{l}k \bar{o}^{\mathrm{Mt}} \\
k o g o-t o^{\mathrm{Kg}}\end{array}$ & $\begin{array}{l}{ }^{*} \text { kopå } \\
(\mathrm{J} 73-74 ; \\
\mathrm{H} \text { 520) } \\
\mathrm{U}{ }^{\star} \text { kopa } \\
\text { (R 180-81) = } \\
{ }^{*} \text { kopå } \\
\text { (S 537: bark) }\end{array}$ \\
\hline $75 b$. & $\dot{s}^{\prime} a r_{\text {Ter }}$ & & & qora $^{\text {Tas }} / \mathrm{E}$ & & & 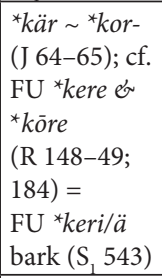 \\
\hline $75 c$. & $t \bar{e} \cdot \eta k_{\mathrm{Le}}$ & & & $\begin{array}{l}\left(t_{\text {tšin }} \Gamma_{\mathrm{D}}^{\mathrm{c}}\right. \\
\text { made } \\
\text { skin })\end{array}$ & & & ${ }^{*}$ cẹjkâ (J 33) \\
\hline $75 \mathrm{~d}$. & $\begin{array}{l}\text { yaja } a_{\mathrm{RNS}} \\
\text { (human } \\
\text { s.) } \\
\end{array}$ & & & & & & $\begin{array}{l}\text { *åjå (J 17; } \\
\text { H 799); } \\
=\# 53 \mathrm{~b}\end{array}$ \\
\hline $\begin{array}{l}76 \mathrm{a} . \\
\text { sleep }\end{array}$ & hônym & \begin{tabular}{|l|} 
koddua- \\
ro'ch \\
koddua- \\
do \\
\end{tabular} & $\begin{array}{l}\text { kundua- } \\
\text { tum }\end{array}$ & $\begin{array}{l}\text { kondak }^{\mathrm{N}} \\
\text { konday- } \\
\text { K,B,Tas }\end{array}$ & $\begin{array}{l}\text { kunollam } \\
\text { kunōlàm }_{\mathrm{D}}\end{array}$ & $\begin{array}{l}\text { xonda- } \\
\text {-štamb } \\
\text { chonda }^{\mathrm{Tg}}\end{array}$ & 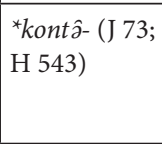 \\
\hline $\begin{array}{l}77 a . \\
\text { small }\end{array}$ & ńüdea & $\begin{array}{l}\text { urea }^{\mathrm{Ch}} \\
\text { judea }^{\mathrm{B}}\end{array}$ & & $\begin{array}{l}\left(y \check{c} e^{N}\right. \\
\text { young, } \\
\text { child })\end{array}$ & & & 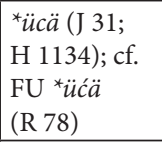 \\
\hline $77 \mathrm{~b}$. & ń/yôloko & & & & & & \\
\hline $77 \mathrm{c}$. & ńuocko & & & & & & \\
\hline $77 \mathrm{~d}$. & manor" ${ }_{\mathrm{NRS}}$ & & & & & & \\
\hline $77 \mathrm{e}$. & & $\begin{array}{l}\text { iblajgu }^{\mathrm{B}} /{ }_{\mathrm{M}} \\
\text { ubulaj- } \\
\text { ku }^{\mathrm{B}} / \\
\text { ublajg }^{\mathrm{K}} /{ }_{\mathrm{M}} \\
\text { ûlaiggu }^{\mathrm{Ch}}\end{array}$ & aligak $_{\mathrm{NRN}}$ & & & & (cf. M 101) \\
\hline $77 f$. & & & sielaku & & & & \\
\hline $77 \mathrm{~g}$. & & & & n'ûnó & & & $\begin{array}{l}{ }^{*} \text { ńuว(j) child } \\
(\mathrm{J} 111 ; \\
\text { DA 1626) }\end{array}$ \\
\hline $77 \mathrm{~h}$. & & & & \begin{tabular}{|l|}
$k e b a^{\mathrm{N}}$, \\
$k i p a^{\mathrm{B}, T a s}$
\end{tabular} & & & \\
\hline $77 \mathrm{i}$. & & & tanolik $\ddot{u}_{\mathrm{NRN}}$ & \begin{tabular}{|l|} 
tâna- \\
$k a^{\check{c}, \mathrm{OO}, \mathrm{K}}$
\end{tabular} & & & $\begin{array}{l}\text { cf. }{ }^{*} \text { tän3- } \\
\text { wenig (J 154) }\end{array}$ \\
\hline
\end{tabular}


Václav Blažek

\begin{tabular}{|c|c|c|c|c|c|c|c|}
\hline gloss & Nenets & Enets & Nganasan & Selkup & Kamas & Mator & etymology \\
\hline $77 \mathrm{j}$. & & & & & $\begin{array}{l}\text { bok } t^{u t} \\
\beta o p t^{2} \\
(+ \text { low, } \\
\text { short })\end{array}$ & & \\
\hline $77 \mathrm{k}$. & & & & & $i d i^{i} e_{\mathrm{D}}^{\prime}$ & & \\
\hline 771. & & & & & $\frac{\ddot{u} \text { dügäa }}{\underline{u d u g g}_{D}}=$ & $\begin{array}{l}\underline{u d z ̌ j u}^{\mathrm{mbut}} \\
{ }^{\mathrm{Mbuj}}\end{array}$ & $\begin{array}{l}\text { < Mo üčü- } \\
\text {-ken } \\
\text { (H 1134) }\end{array}$ \\
\hline $\begin{array}{l}\text { 78a. } \\
\text { smoke } \\
\text { n. }\end{array}$ & $\begin{array}{l}\text { jâke } \\
\text { jáki } i_{\text {Adelung }}\end{array}$ & $\begin{array}{l}j a ́ k i^{\mathrm{Ch}} \\
d^{\prime} a k a^{\mathrm{B}} / \mathrm{Te}\end{array}$ & \begin{tabular}{|l} 
(kíndo \\
djakága $_{\mathrm{Te}}$ \\
smell of \\
smoke) \\
\end{tabular} & $\begin{array}{l}\text { ?kačka }{ }^{\mathrm{N}} \\
\text { ?kacko- } \\
\mathrm{K}, \mathrm{c}, \mathrm{OO}\end{array}$ & & & *jäckâ- (J 39) \\
\hline $78 \mathrm{~b}$. & $\begin{array}{l}\text { śun } \\
(+ \text { steam })\end{array}$ & $\begin{array}{l}\text { śuddo }{ }^{\mathrm{Ch}} \\
\text { šudu }{ }^{\mathrm{K}} / \mathrm{M} \\
\text { šudru }{ }_{\mathrm{Gl}}^{\mathrm{B}}\end{array}$ & kinta & sümde ${ }^{\mathrm{NP}}$ & $\begin{array}{l}\text { schunjú } \\
\text { Adelung }\end{array}$ & $\begin{array}{l}\text { kjund }^{\mathrm{Mt}} \\
\text { kundo }^{\mathrm{Tg}}\end{array}$ & 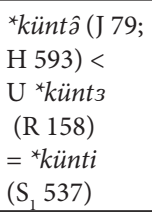 \\
\hline $78 \mathrm{c}$. & $\begin{array}{l}\text { (pur" fire } \\
\text { against } \\
\text { mosqui- } \\
\text { tos) }\end{array}$ & & & $\begin{array}{l}\text { purga }^{\mathrm{B} \text {,Tas }} \\
= \\
\text { purqi }^{\mathrm{Tas}} / \mathrm{E} \\
\text { (cf. DA } \\
661 \text { ) } \\
\end{array}$ & & & $\begin{array}{l}{ }^{*} \text { pur- }(\mathrm{J} \mathrm{131)} \\
<\mathrm{U}{ }^{*} \text { purki } \\
\left(\mathrm{A}_{1} 25\right) \text { or } \\
\text { lw. from Kh } \\
\text { pŏrki? }\end{array}$ \\
\hline $78 \mathrm{~d}$. & & & & \begin{tabular}{|l|}
$k a \bar{a} m$ \\
$-d \partial^{\mathrm{KM}} / \mathrm{DA}$ \\
\end{tabular} & & & $\begin{array}{l}{ }^{*} k a ̈ m t \hat{~ s o o t ~} \\
\text { (J 64) }\end{array}$ \\
\hline $78 \mathrm{e}$. & & & & & $\begin{array}{l}\underline{b e r}=\underline{b e r} \\
\underline{\text { bor }}, \underline{\text { bôr }} \underline{\mathrm{D}}\end{array}$ & & $\begin{array}{l}\text { cf. Tat. Tobol } \\
\text { byr } \\
\text { soot (Jo 90, } \\
96 \text { ) }\end{array}$ \\
\hline $78 \mathrm{f}$. & & & & & $k^{\prime} a ̊ m n u_{\mathrm{D}}^{\prime}$ & & \\
\hline $\begin{array}{l}79 a . \\
\text { stand }\end{array}$ & $n \hat{u} d m$ & $\begin{array}{l}\text { nôaro'Ch } \\
\text { nôado'B }^{\text {'B }}\end{array}$ & nandetm & $\begin{array}{l}\text { nènak }^{\mathrm{N}} \\
\text { nènay }^{\mathrm{K},-} \\
\text { Mo,oo } \\
\text { leyay }^{\mathrm{C}} \\
\end{array}$ & $\begin{array}{l}\text { nugam = } \\
\text { nul'äm, } \\
\text { nōläm }_{\mathrm{D}}\end{array}$ & $\begin{array}{l}\text { nemyn- } \\
- \text { dams }^{\mathrm{Mt}}\end{array}$ & $\begin{array}{l}{ }^{*} n u-\sim{ }^{*} n i- \\
\text { (J 104; } \\
\text { H 735) }\end{array}$ \\
\hline $79 b$. & $\begin{array}{l}\text { (tōottì̃è } \\
\text { stellen) }\end{array}$ & & & $\begin{array}{l}\text { totti-qo- } \\
\text { Tas/ } \\
\text { če/očči- } \\
-{ }_{-} \mathrm{Ty} / \mathrm{DA} \\
\end{array}$ & & & $\begin{array}{l}\text { cf. DA 1064, } \\
1084\end{array}$ \\
\hline $\begin{array}{l}80 a . \\
\text { star }\end{array}$ & $n u m g \hat{y}$ & & & & & & $\begin{array}{l}\text { cf. *num sky, } \\
\text { god } \\
\text { (J 104; } \\
\text { H 782) < } \\
\mathrm{U}^{*} n u-m 3 \\
\text { (R 308) }\end{array}$ \\
\hline $80 b$. & & $\begin{array}{l}\text { foreseo }^{\mathrm{Ch}} \\
\text { fadese }^{\mathrm{B}}\end{array}$ & $\begin{array}{l}\text { fatajea }= \\
\text { hotadio- } \\
\text { NRN }\end{array}$ & & & & \\
\hline
\end{tabular}


On the classification of the Samoyedic languages

\begin{tabular}{|c|c|c|c|c|c|c|c|}
\hline gloss & Nenets & Enets & Nganasan & Selkup & Kamas & Mator & etymology \\
\hline $80 \mathrm{c}$. & & & & \begin{tabular}{|l}
$k e s ̌ k a^{\mathrm{N}}$ \\
$k u e s ̌ k a^{\mathrm{B}, T a s}$ \\
$k e s a \eta-$ \\
$k a^{\mathrm{K}, \mathrm{C}, \mathrm{NP}}$
\end{tabular} & $\begin{array}{l}\text { khinzi- } \\
\text { gäi }= \\
k^{\prime} \hat{\imath} n d^{2} z_{i g e i_{\mathrm{D}}}\end{array}$ & $\begin{array}{l}\text { kindži- } \\
k e j^{\mathrm{Mt}}\end{array}$ & $\begin{array}{l}\text { *kins3-(kåjâ) } \\
\text { (JJ 263; } \\
\text { H 489)< } \\
\text { U *kuńć3 } \\
\text { (R 210) = } \\
{ }^{*} k \text { kunśå } \\
\text { (S, 537) }\end{array}$ \\
\hline $\begin{array}{l}81 \mathrm{a} . \\
\text { stone }\end{array}$ & pae & $f \hat{u}^{\mathrm{Ch}}, f u^{\mathrm{B}}$ & fala & $\begin{array}{l}p \ddot{o}^{\mathrm{N}}, \\
p \ddot{u}^{\mathrm{K}, \mathrm{OO},} \\
p \overline{\ddot{u}}_{<}^{\mathrm{B}, \mathrm{Tas}}\end{array}$ & $p h i=p^{\prime} \bar{i}_{\mathrm{D}}$ & hil $\ddot{a ̆}^{\mathrm{Mt}}$ & $\begin{array}{l}{ }^{*} \text { pâj } j \text { (J 112; } \\
\text { H 298)< } \\
\text { U * pije } \\
\text { (R 378) }\end{array}$ \\
\hline $\begin{array}{l}82 a . \\
\text { sun }\end{array}$ & $\begin{array}{l}\text { hâjer, } \\
\text { haijer }\end{array}$ & $\begin{array}{l}\text { kaija }^{\mathrm{Ch}} \\
\text { kaja }^{\mathrm{B}} / \mathrm{Te} \\
\text { kāja }^{\mathrm{K}} / \mathrm{Pu} \\
\text { kājer }^{\mathrm{K}} / \mathrm{Pu}\end{array}$ & kou & $\begin{array}{l}\left(q e c{ }^{\mathrm{Tas}} / \mathrm{E}\right. \\
\text { heat })\end{array}$ & $\begin{array}{l}\text { kuja }= \\
k^{c} u i a_{\mathrm{D}}\end{array}$ & $\begin{array}{l}\text { kajja }^{\mathrm{Mt}} \\
\text { xaja }^{\mathrm{Tg}}\end{array}$ & $\begin{array}{l}{ }^{*} k a ̊ j a ̊(\mathrm{~J} \mathrm{58;} \mathrm{H} \\
389)< \\
\mathrm{U}{ }^{*} k o j e \\
(\mathrm{R} 167)= \\
{ }^{*} k a ̊ j a ̊\left(\mathrm{~S}_{1} 538\right)\end{array}$ \\
\hline $82 b$. & $\begin{array}{l}\text { (jâlea } \\
\text { day, light) }\end{array}$ & $\begin{array}{l}\left(\text { jeré }^{\mathrm{Ch}}\right. \\
\left.\text { jere }^{\mathrm{B}} \text { day }\right)\end{array}$ & (jale day) & 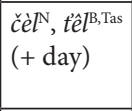 & 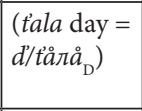 & & 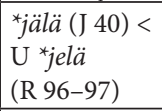 \\
\hline $\begin{array}{l}83 a . \\
\text { swim }\end{array}$ & $\eta \bar{u}-\dot{s}_{\mathrm{Le}}$ & & & $\begin{array}{l}\hat{u} r n a k^{\mathrm{N}} \\
\hat{u} r n a \eta^{\mathrm{K}, \mathrm{O}-} \\
\mathrm{O}, \mathrm{B}, \mathrm{Tas}\end{array}$ & & $\begin{array}{l}{ }^{*} u-\text { or } \\
{ }^{*} \bar{u}-\mathrm{Kg} \\
t s c h a- \\
\text { gand- } \\
\text { uùsugur- } \\
\text { jana } \\
\text { I swim in } \\
\text { a river }\end{array}$ & $\begin{array}{l}{ }^{*} u-(\mathrm{J} 29 ; \\
\mathrm{H} 1108)< \\
\mathrm{U}{ }^{*} u j e-\sim \\
{ }^{*} \text { oje- } \\
(\mathrm{R} 542)= \\
{ }^{*} u x i- \\
\left(\mathrm{S}_{1} 536\right)\end{array}$ \\
\hline $83 b$. & $\begin{array}{l}\text { oûlim, } \\
\text { ôlym }\end{array}$ & & & & & & \\
\hline $83 \mathrm{c}$. & hûlydm & & & & & & \\
\hline $83 \mathrm{~d}$. & hattadm & & & & & & \\
\hline $83 \mathrm{e}$. & $\begin{array}{l}\text { śam- } \\
\text { beadm }\end{array}$ & & & $\begin{array}{l}\text { kāam- } \\
\text { par- }^{\mathrm{KM}} / \mathrm{DA}\end{array}$ & & & cf. DA 1835 \\
\hline $83 \mathrm{f}$. & & $\begin{array}{l}\text { be'ero } \\
\text { be' }^{\mathrm{Ch}} \\
\end{array}$ & & & & & \\
\hline $83 \mathrm{~g}$. & & $\begin{array}{l}\text { beheyedo }^{\mathrm{B}} \\
\text { beheyero }^{\mathrm{Ch}}\end{array}$ & & & & & \\
\hline $83 \mathrm{~h}$. & $\dot{r}^{\prime} \dot{a} m p a s_{\mathrm{Le}}$ & & $\begin{array}{l}\text { jambi'em } \\
= \\
\text { d'am- } \\
\text { biśi }{ }_{\mathrm{NRN}}\end{array}$ & $\begin{array}{l}\text { (lämpi- } \\
\text { la } a^{\text {Tas} / E} \\
\text { Schwim- } \\
\text { mer am } \\
\text { Netz) }\end{array}$ & & & *lämpâ (J 82) \\
\hline $83 i$. & & & hoofirsi $_{\mathrm{NRN}}$ & \begin{tabular}{|l|} 
kurak $^{\mathrm{N}}$ \\
kûrem- \\
bay $^{\text {Tas }}$ \\
\end{tabular} & & & \\
\hline $83 \mathrm{j}$. & & & kudüs $_{\mathrm{NRN}}$ & & & & \\
\hline
\end{tabular}


Václav Blažek

\begin{tabular}{|c|c|c|c|c|c|c|c|}
\hline gloss & Nenets & Enets & Nganasan & Selkup & Kamas & Mator & etymology \\
\hline $83 \mathrm{k}$. & & & & $\begin{array}{l}\text { pänžay } \\
\text { päntetay }^{\mathrm{B}} \\
\end{array}$ & $\begin{array}{l}\text { pha(')lam = } \\
p^{(} \hat{a}^{\prime} l^{\prime} a m_{\mathrm{D}} \\
\end{array}$ & & \\
\hline 831. & & & & & & $\begin{array}{l}\text { tadelb- } \\
\text { namb }^{\mathrm{Mt}}\end{array}$ & (M 934) \\
\hline $\begin{array}{l}84 a . \\
\text { tail }\end{array}$ & tae(u)wa & & tájbu & $\begin{array}{l}\text { tal }^{\mathrm{N}} \\
\text { tald }^{\mathrm{K}}\end{array}$ & $\begin{array}{l}\text { thîma }= \\
\text { tš̌̀ima }_{\mathrm{D}}\end{array}$ & dajba ${ }^{\mathrm{Mt}}$ & $\begin{array}{l}{ }^{*} t_{1} \text { åjwå } \\
(\mathrm{J} 150 ; \mathrm{H} \\
943)< \\
\mathrm{U}^{*} \text { sejpä } \\
(\mathrm{R} 438)\end{array}$ \\
\hline $84 b$. & sańa' & $\begin{array}{l}\text { (sóddaki } \\
\text { tail of } \\
\text { birds) }\end{array}$ & $\begin{array}{l}\text { (santu' } \\
\text { tail of } \\
\text { birds) }\end{array}$ & & & & \\
\hline $84 c$. & panco & & & & & & $\begin{array}{l}\text { cf. Sm * }{ }^{*} a ̊ n c o ̂ \\
\text { lower edge } \\
\text { (J 116)< } \\
U^{*}{ }^{*} \text { ponči tail } \\
(\mathrm{R} 394-95, \\
735 ; \mathrm{S}_{1} 547 ; \\
\left.\mathrm{A}_{2} 23\right)\end{array}$ \\
\hline & & $\begin{array}{l}\text { batu'o } \mathrm{Ch}^{\mathrm{Ch}} \\
\text { batu' }^{\mathrm{B}} \mathrm{o}_{\mathrm{Te}}\end{array}$ & batụ'a & & & & \\
\hline $84 \mathrm{~d}$. & & & & $\begin{array}{l}\text { mäčă } \ddot{a}^{\mathrm{B}} \\
\text { mät } \ddot{a}^{\mathrm{Tas}}\end{array}$ & & & $\begin{array}{l}<\mathrm{U}{ }^{*} \text { mäńćs } \\
{ }^{*} \text { mäćs } \\
(\mathrm{R} 268)\end{array}$ \\
\hline $84 \mathrm{e}$. & & & & & $\begin{array}{l}\frac{\text { katen }}{\mathrm{D}} \\
(+ \text { Arsch) }\end{array}$ & & $\begin{array}{l}<\text { Tat. kötän } \\
\text { id. }\end{array}$ \\
\hline $\begin{array}{l}85 a . \\
\text { that }\end{array}$ & taky & & & $\left(t e^{\mathrm{Tas}} / \mathrm{E}\right.$ he $)$ & & & ${ }^{*} t^{\prime} a ̊-(\mathrm{J} 150)$ \\
\hline $85 a a$. & tănij $_{\mathrm{Te}}$ & $\begin{array}{l}\text { (tonea } \\
\text { es gibt) }\end{array}$ & tanie & & & & $\begin{array}{l}{ }^{*} t \hat{\jmath}-n \ddot{a}- \\
(\mathrm{J} 144)< \\
\mathrm{U}^{*} t a+{ }^{*} n \ddot{a} \\
(\mathrm{R} 505,300)\end{array}$ \\
\hline 85 aaa. & $\begin{array}{l}\text { tiky } \\
\text { der dort) }\end{array}$ & $t i k \varepsilon_{\mathrm{Te}}$ & & $\begin{array}{l}\left(t i^{\mathrm{Tas}} / \mathrm{E}\right. \\
\text { now }) \\
t i n a^{\mathrm{Tas}} / \mathrm{E} \\
(+ \text { this })\end{array}$ & $d \partial_{\mathrm{D}}(+\mathrm{he})$ & $\begin{array}{l}t i n b^{\mathrm{Mt}} \\
\left(t y^{\mathrm{Mt}} \mathrm{he}\right)\end{array}$ & $\begin{array}{l}{ }^{*} t i(-)(\mathrm{J} 160 ; \\
\mathrm{H} 1006, \\
1022)< \\
\left.\mathrm{U}^{*} t \ddot{\prime} /{ }^{*} t e\right)^{*} t i \\
(\mathrm{R} 513-14)\end{array}$ \\
\hline $\begin{array}{l}85 \mathrm{~b} .= \\
86 \mathrm{c} .\end{array}$ & $n \bar{e} r_{\mathrm{Le}}$ & & & & & & $\begin{array}{l}{ }^{*} n 3(-) \\
(\mathrm{J} 105)< \\
\mathrm{U}^{*} n \ddot{a} /^{*} n e^{*}- \\
n i(\mathrm{R} 301)\end{array}$ \\
\hline $85 \mathrm{c}$. & & sedeo & & & & & $\begin{array}{l}\text { cf. } \operatorname{sed}^{\mathrm{B} /} \mathrm{Te} \\
\text { who? }\end{array}$ \\
\hline $85 \mathrm{~d}$. & & $i n e^{\mathrm{B}} /{ }_{\mathrm{Pr}}$ & & & $\left(\bar{i} d_{D}^{\partial}\right.$ this $)$ & $\begin{array}{l}\left(\text { idichaiin }^{\mathrm{Mt}}\right. \\
\text { vorgestern })\end{array}$ & $\begin{array}{l}{ }^{*} i-(\mathrm{J} \mathrm{26} \\
\text { H 336) }\end{array}$ \\
\hline
\end{tabular}


On the classification of the Samoyedic languages

\begin{tabular}{|c|c|c|c|c|c|c|c|}
\hline gloss & Nenets & Enets & Nganasan & Selkup & Kamas & Mator & etymology \\
\hline $\begin{array}{l}86 a . \\
\text { this }\end{array}$ & & & takâ & & šo & & \begin{tabular}{|l}
${ }^{*} t \ddot{a}-{ }^{*}{ }^{*} t e-$ \\
$(\mathrm{J} \mathrm{152)}<$ \\
$\mathrm{U}^{\star}{ }^{\star} t \ddot{a} /{ }^{*}{ }^{\star} t e{ }^{*} t i$ \\
$(\mathrm{R} 513-14)$
\end{tabular} \\
\hline 86aa. & $\begin{array}{l}\left(\text { tat }_{\mathrm{Le}}\right. \\
\text { then })\end{array}$ & tohonô & $\begin{array}{l}\text { (tanday } \\
\text { dorthin) }\end{array}$ & $t o=t a^{\mathrm{Tas}} / \mathrm{E}$ & $d a$ & $\begin{array}{l}\left(\text { dana }^{\mathrm{Mt}}\right. \\
\text { there })\end{array}$ & $\begin{array}{l}{ }^{*} t \hat{\partial}-(\mathrm{J} 144 ; \\
\mathrm{H} 954)< \\
\mathrm{U}^{*} t a(\mathrm{R} 505)\end{array}$ \\
\hline 86aaa. & $\begin{array}{l}\text { tuky }= \\
t^{\prime} u k u_{\mathrm{RNS}}\end{array}$ & & & & $d \ddot{u}$ & & $\begin{array}{l}{ }^{*} \text { tü- }(\mathrm{J} 167 ; \\
\mathrm{H} 1075 \\
\text { rejects to add } \\
\text { Mt tugokz } \\
\text { soviel) }\end{array}$ \\
\hline $86 \mathrm{~b}$. & & $\begin{array}{l}\text { dat. eo' } \\
\text { der. eke/ } \\
o^{\mathrm{Ch}} \\
\varepsilon k k \varepsilon^{\mathrm{B}} / \mathrm{Te} \\
\varepsilon k e^{\mathrm{K}} / \mathrm{M}\end{array}$ & yamay & $\begin{array}{l}\text { (ompä } \\
\text { Tas/ } \\
\text { now) }\end{array}$ & & & $\begin{array}{l}{ }^{*} \ddot{m} \text { - }(\mathrm{J} 19)< \\
\mathrm{U}{ }^{*} e(\mathrm{R} 67) ; \\
\mathrm{H} 644 \text { rejects } \\
\text { to add } \mathrm{Mt} \\
\text { mákaia today } \\
\text { : Hu ma id. }\end{array}$ \\
\hline $86 \mathrm{c}$. & $\begin{array}{l}\left(n \bar{e} r_{L e}\right. \\
\text { that) }\end{array}$ & & & na & & & $\begin{array}{l}{ }^{*} n 3(-)(\mathrm{J} \mathrm{105}) \\
<\mathrm{U}{ }^{*} n o \\
(\mathrm{R} 306)\end{array}$ \\
\hline $86 \mathrm{~d}$. & & & & & $\bar{i} d_{D}{ }_{D}$ & & $=\# 85 \mathrm{~d}$ \\
\hline $\begin{array}{l}87 \mathrm{a} . \\
\text { thou }\end{array}$ & & $\begin{array}{l}\text { todì } \\
\left({ }^{*} t \partial \hat{n}-t a ̈\right)\end{array}$ & tannay & $\tan / t$ & $\begin{array}{l}\text { than }= \\
t^{\prime} a n, \\
t^{\prime} a ̊ n_{\mathrm{D}}\end{array}$ & $\tan \sigma^{\mathrm{Mt}}$ & $\begin{array}{l}{ }^{*} \text { tân }(\mathrm{J} \mathrm{147)}< \\
\mathrm{U}^{*} \text { tun } \\
\text { (JJ 232; } \\
\text { R 539) }\end{array}$ \\
\hline $87 \mathrm{~b}$. & $\begin{array}{l}\text { pudar }= \\
\text { pydăr }{ }_{\mathrm{NRS}}\end{array}$ & & & & & $\begin{array}{l}\text { (xudu- } \\
\text { self })\end{array}$ & $\begin{array}{l}\text { from puda he } \\
\text { (GS 342); cf. } \\
\text { pyd } d_{\mathrm{NRS}} \text { self; } \\
\text { body < }{ }^{*} \text { puca } \\
\text { (J 129-30; } \\
\text { H 321) } \\
\end{array}$ \\
\hline $87 \mathrm{c}$. & & $\begin{array}{l}\underline{\bar{u}}^{\mathrm{B}} / \mathrm{Te} \\
\underline{\bar{u}}^{\mathrm{K}} /{ }_{\mathrm{M}}, \underline{u}^{\mathrm{K}} / \mathrm{Pu}\end{array}$ & & & & & $\begin{array}{l}\text { (M 230) } \\
\text { cf. Ket } \bar{u} \text { thou }\end{array}$ \\
\hline $\begin{array}{l}88 \mathrm{a} . \\
\text { ton- } \\
\text { gue }\end{array}$ & $\begin{array}{l}\text { ńâmi } \\
\text { Obd. } \\
\text { ńāmū = } \\
\text { ńamju }\end{array}$ & & & & & & $\begin{array}{l}\text { R 314: ńā? } \\
\text { mouth + } \\
{ }^{*} \text { mū } \text { Inne- } \\
\text { res; not from } \\
{ }^{*} \text { n̈älmä } \\
\end{array}$ \\
\hline $88 \mathrm{~b}$. & $s i \dot{e}_{\mathrm{Le}}$ & $\begin{array}{l}\text { sióro }^{\mathrm{Ch}} \\
\text { sioro }^{\mathrm{B}}\end{array}$ & sieja & $\begin{array}{l}s e^{\mathrm{N}}, \check{s}^{\text {Tas }}, \\
\check{s} \hat{l}^{\mathrm{B}}\end{array}$ & $\begin{array}{l}\text { šikä = } \\
\text { šak } \partial_{\mathrm{D}}\end{array}$ & $\begin{array}{l}\text { ki, kis- } \\
\text { cht } \ddot{a}^{\mathrm{Mt}} \\
g e i^{\mathrm{Kg}}\end{array}$ & $\begin{array}{l}{ }^{*} k e a ̂ j j(\mathrm{~J} 66 ; \\
\mathrm{H} 450)< \\
\mathrm{U}{ }^{\star} k \bar{e} l e \\
(\mathrm{R} 144)= \\
{ }^{*} \text { käxli } \\
(\mathrm{S}, 538)\end{array}$ \\
\hline
\end{tabular}


Václav Blažek

\begin{tabular}{|c|c|c|c|c|c|c|c|}
\hline gloss & Nenets & Enets & Nganasan & Selkup & Kamas & Mator & etymology \\
\hline $\begin{array}{l}89 \mathrm{a} . \\
\text { tooth }\end{array}$ & $\begin{array}{l}\text { tibea, } \\
\text { tiwie }\end{array}$ & 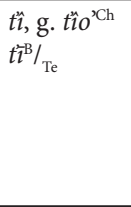 & timi & $\begin{array}{l}\text { tim }^{\mathrm{B}, \mathrm{Tas}}, \\
\operatorname{tiu}^{\mathrm{K}} \\
\text { teu }^{\mathrm{MO}} \\
\check{c} e u^{\mathrm{C}}\end{array}$ & $\begin{array}{l}\text { thimä }= \\
t^{\prime} i m \varepsilon_{\mathrm{D}}\end{array}$ & $\begin{array}{l}\text { tyme } \\
\text { dimi-da }\end{array}$ & $\begin{array}{l}{ }^{*} \text { timä (J 163; } \\
\mathrm{H} 1019)< \\
\mathrm{U}{ }^{*} \operatorname{sew}(i)-m \ddot{~} \\
:{ }^{*} \operatorname{sewi} \text { - eat } \\
\left(\mathrm{A}_{1} 34-35 ;\right. \\
\text { R 440; S 548) }\end{array}$ \\
\hline $\begin{array}{l}\text { 90a. } \\
\text { tree }\end{array}$ & pea & $f e^{\mathrm{Ch}}, f e^{\mathrm{B}}$ & $f \grave{a}$ & $\begin{array}{l}p o^{\mathrm{N}}, \\
p \hat{u}^{\mathrm{B}, \mathrm{Tas}}\end{array}$ & pha & $h \ddot{a} h^{\mathrm{Mt}}$ & $\begin{array}{l}{ }^{*} p \ddot{a}(\mathrm{~J} 117 ; \\
\mathrm{H} 267)< \\
\mathrm{U}{ }^{*} \text { puwe } \\
(\mathrm{R} 410)= \\
{ }^{*} \text { pu/o/åxi் } / i \\
\left(\mathrm{~S}_{1} 539\right)\end{array}$ \\
\hline $\begin{array}{l}\text { 91a. } \\
\text { two }\end{array}$ & side & $\begin{array}{l}\text { sire }^{\mathrm{Ch}}, \\
\text { side }^{\mathrm{B}}\end{array}$ & $s i t i=s i^{\prime} i^{\mathrm{M}}$ & site, site & šide & $k i d d \ddot{a}^{\mathrm{Mt}}$ & $\begin{array}{l}{ }^{*} \text { kitä }(\mathrm{J} 71 ; \\
\mathrm{H} 481)< \\
\mathrm{U}{ }^{*} k a k t a{ }^{*}- \\
\text { käktä } \\
(\mathrm{R} 118-19)= \\
{ }^{*} \text { kektä } \\
(\mathrm{S}, 537)\end{array}$ \\
\hline $\begin{array}{l}92 \mathrm{a} . \\
\text { walk }\end{array}$ & jâdam & 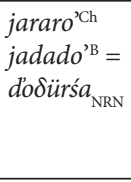 & joturum & $\begin{array}{l}\text { kuttarna- } \\
\eta^{\text {Kar }} \\
\text { kuttar- } \\
-q o^{\text {Tas }} / \mathrm{E}\end{array}$ & & $\begin{array}{l}\text { dscháda }{ }^{\mathrm{Mt}} \\
\text { dscha- } \\
\text { dim }^{\mathrm{Tg}}\end{array}$ & $\begin{array}{l}\text { *jåtô-(j-r-) } \\
(\mathrm{J} 38-39 ; \\
\text { H 164, 166)< } \\
\text { U *juta- } \\
\text { (R 106) }\end{array}$ \\
\hline $92 \mathrm{~b}$. & hajeadm & & & & & $\begin{array}{l}\text { gajty- } \\
\text { gams }^{\mathrm{Mt}}\end{array}$ & $\begin{array}{l}{ }^{*} \text { kâj- (J 51; } \\
\text { H 384: var.: } \\
\left.{ }^{*} \text { kań-, }{ }^{*} k a j-\right)\end{array}$ \\
\hline $92 c$. & & $\begin{array}{l}\left(\text { kańiš }^{\mathrm{B}} / \mathrm{Te}\right. \\
\text { (weg) } \\
\text { gehen) }\end{array}$ & konidi & $\begin{array}{l}\text { qen-qo- } \\
\text { Tas/ }\end{array}$ & $\begin{array}{l}\text { kanda- } \\
\text { gam }^{\mathrm{Kb}}\end{array}$ & $\begin{array}{l}\left(g n^{\mathrm{Kg}}\right. \\
\text { уйди!) }\end{array}$ & $\begin{array}{l}{ }^{*} k a ̊(\hat{)}) n- \\
(\mathrm{J} \mathrm{59;} \mathrm{H} \mathrm{413);} \\
\text { cf. U }{ }^{*} \text { kanta } \\
\text { carry (R 124; } \\
\text { S 24) }\end{array}$ \\
\hline 92d. & $\min \dot{z}_{\mathrm{RNS}}$ & & $\begin{array}{l}\text { méndem } \\
= \\
\text { minsin }_{\mathrm{NRN}}\end{array}$ & & $\begin{array}{l}\text { miyäm }= \\
\text { måggem }_{\mathrm{D}}\end{array}$ & & $\begin{array}{l}{ }^{*} \text { min- } \\
\text { (J 94; H } 627 \\
\text { excudes Mt } \\
\text { myngaems } \\
\text { I leave) < } \\
\mathrm{U}^{*} \text { mene } \\
\text { (R 272) } \\
={ }^{*} \text { meni- } \\
(\mathrm{S}, 538)\end{array}$ \\
\hline $92 \mathrm{e}$. & & muosiro $^{\mathrm{Ch}}$ & & & & & \\
\hline $92 \mathrm{f}$. & & & čüüd ${ }^{\prime} a_{\mathrm{NRN}}$ & & & & \\
\hline 92g. & & & $\begin{array}{l}\text { heditisi- } \\
\text { NRN }\end{array}$ & & & & \\
\hline $92 \mathrm{~h}$. & & & & $\begin{array}{l}\text { pald'uay }^{\mathrm{K}} \\
\text { pald'uwa- } \\
\eta^{\mathrm{NP}} \\
\end{array}$ & & & \\
\hline
\end{tabular}


On the classification of the Samoyedic languages

\begin{tabular}{|c|c|c|c|c|c|c|c|}
\hline gloss & Nenets & Enets & Nganasan & Selkup & Kamas & Mator & etymology \\
\hline $92 \mathrm{i}$. & & & & \begin{tabular}{|l|} 
werkka- \\
$\eta^{\mathrm{C}, \mathrm{OOO}}$
\end{tabular} & & & \\
\hline $92 \mathrm{j}$. & & & & iččir-qo & & & \\
\hline $92 \mathrm{k}$. & & & & $\begin{array}{l}\text { qaltir- } \\
\text { kaltiri-qo }\end{array}$ & & & \\
\hline 921. & & & & $q o s_{-q o}$ & & & \\
\hline $92 \mathrm{~m}$. & & & & & $\begin{array}{l}\text { båd }{ }^{\prime} l a \dot{m} m_{\mathrm{D}} \\
\text { (+ bring) }\end{array}$ & & \\
\hline $92 n$. & & & & & $\begin{array}{l}k^{(\bullet)} \text { åлла̊m, } \\
\text { ха̊лла̊m } \\
\end{array}$ & & $\begin{array}{l}<\mathrm{U}^{*}{ }^{*} \text { kulke- } \\
\text { (R 198) }\end{array}$ \\
\hline 920. & & & & & milem $_{\mathrm{D}}$ & & \\
\hline 92p. & & & & & $\begin{array}{l}\text { šōnuyam, } \\
\text { šōnam- } \\
\text { nam }_{\mathrm{D}} \\
\end{array}$ & & \\
\hline $92 \mathrm{q}$. & & & & & $\begin{array}{l}\text { t'ōonníim, } \\
\text { t'ōnu'läam } \\
\end{array}$ & & \\
\hline $\begin{array}{l}\text { 93a. } \\
\text { warm }\end{array}$ & $\begin{array}{l}j i b i, j \hat{\imath} p i, \\
j u b a, j y b a\end{array}$ & 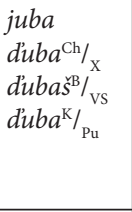 & & & $\begin{array}{l}(\text { dü- } \text { melt } \\
\{\text { of ice }\})\end{array}$ & $\begin{array}{l}\text { tschúha } \\
\text { g.Mt }\end{array}$ & $\begin{array}{l}{ }^{*} j u(\hat{\partial})-p a ̊: \\
\star_{j u(\hat{\partial})-} \\
\text { become } \\
\text { warm, weak } \\
\text { (J 47-48; } \\
\text { H 231) }\end{array}$ \\
\hline $93 b$. & & & $\begin{array}{l}\text { hejbi } i_{\mathrm{NRN}} \\
\text { (fea- } \\
\text { bem'am } \\
\text { sich er- } \\
\text { wärmen) }\end{array}$ & $\begin{array}{l}p \ddot{o}^{\mathrm{N}}, \\
p \ddot{u}^{\mathrm{K}, \mathrm{B}, \text { Tas }}\end{array}$ & & & $\begin{array}{l}{ }^{*} \text { pejwä } \\
\text { (J 120); } \\
\text { cf. FU *päjwä } \\
\text { fire (R 360; } \\
\text { S 34) }\end{array}$ \\
\hline $93 \mathrm{c}$. & & & núnay & & & & \\
\hline 93d. & & & $\begin{array}{l}\text { fekutea }= \\
\underline{\text { hékutaku }}_{\mathrm{x}}\end{array}$ & & & & $\begin{array}{l}<\operatorname{Tn}{ }^{*} p e k u \\
(\text { AX 146-47) }\end{array}$ \\
\hline & & & $h /$ kolśuo $_{\mathrm{KS}}$ & & & & \\
\hline $93 \mathrm{e}$. & & & & & 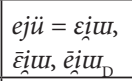 & & \\
\hline $93 \mathrm{f}$. & $\begin{array}{l}\text { (jüepp } \bar{a}_{\mathrm{Le}} \\
\text { be hot) }\end{array}$ & $\begin{array}{l}\left(j e ́ f i^{\mathrm{B}}, e ́ f i^{\mathrm{ch}}\right. \\
\text { be hot) }\end{array}$ & & $\begin{array}{l}(\check{c} a \bar{a} p(p) \\
i-q o^{\text {Tas }} / \mathrm{E} \\
\text { burn })\end{array}$ & $\begin{array}{l}t^{\prime}(\check{s}) i^{u t} g \mathcal{E} \cdot \\
d^{\prime} z ̌ i b u u g \varepsilon_{\mathrm{D}}\end{array}$ & džobuka ${ }^{\mathrm{Mt}}$ & 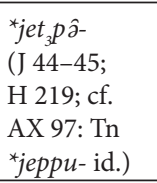 \\
\hline $\begin{array}{l}94 \mathrm{a} . \\
\text { water }\end{array}$ & $j i, w i t^{\text {Knd }}$ & $b i^{\mathrm{Ch}}, b i^{\mathrm{B}}$ & $b \hat{e}$ & $\ddot{u} t$, öt $t^{\mathrm{N}, \mathrm{B}, \mathrm{Tas}}$ & $b \ddot{u}=b u u_{\mathrm{D}}$ & $b u^{\mathrm{Mt}, \mathrm{Tg}, \mathrm{Kg}}$ & $\begin{array}{l}{ }^{*} \text { wit }(\mathrm{J} \mathrm{176;} \\
\mathrm{H} 143)< \\
\mathrm{U}{ }^{*} \text { wete } \\
(\mathrm{R} 570)= \\
{ }^{*} \text { weti }\left(\mathrm{S}_{1} 541\right)\end{array}$ \\
\hline
\end{tabular}


Václav Blažek

\begin{tabular}{|c|c|c|c|c|c|c|c|}
\hline gloss & Nenets & Enets & Nganasan & Selkup & Kamas & Mator & etymology \\
\hline $\begin{array}{l}95 a . \\
\text { we }\end{array}$ & & & mêy & $m \hat{e}, m \hat{\imath}$ & $m i$ & mendä & $\begin{array}{l}{ }^{*} m e-(N /-t) \\
(\mathrm{J} \mathrm{91;} \mathrm{H} \mathrm{655)} \\
<\mathrm{U}{ }^{*} m e / \\
{ }^{*} m \ddot{~}(-N)(\mathrm{cf} . \\
\text { R 294-95) }\end{array}$ \\
\hline 95ab. & mańa' & $\begin{array}{l}\text { modi' } \\
\text { modina- } \\
\text { 'B/ } / \mathrm{Pr} \\
\text { modina- } \\
\mathrm{Ch} / \mathrm{Su}\end{array}$ & & & & & $\begin{array}{l}{ }^{*} m a ̂ n-t \ddot{a}-(t) \\
(J \text { 86) }\end{array}$ \\
\hline $\begin{array}{l}96 a . \\
\text { what }\end{array}$ & yamgy & $\begin{array}{l}\text { awuo } \\
a b b u a^{\mathrm{B}} / \mathrm{Pr} \\
o b u^{\mathrm{B}} / \mathrm{Te}\end{array}$ & & & \begin{tabular}{l|}
$\ddot{u} m b i=$ \\
$m b i, a m b i_{\mathrm{D}}$ \\
cf. šombi \\
\end{tabular} & $\begin{array}{l}\text { (amgan-Kg } \\
\text { why) }\end{array}$ & $\begin{array}{l}\text { *âm- (J 15; } \\
\text { H 34) }\end{array}$ \\
\hline $96 \mathrm{~b}$. & $\begin{array}{l}\left(m \bar{i}_{\mathrm{Le}}\right. \\
\text { etwas } \\
\text { zu essen })\end{array}$ & $m \hat{\imath}^{\mathrm{C} h}$ & $m \hat{a}$ & $\begin{array}{l}\text { (mi } \\
\text { thing) }\end{array}$ & $\begin{array}{l}\left(m o_{\mathrm{D}}\right. \\
\text { weshalb) }\end{array}$ & $m a^{-\mathrm{Kg}}$ & $\begin{array}{l}{ }^{*} \text { me }(\mathrm{J} \mathrm{91;} \\
\mathrm{H} 633)< \\
\mathrm{U}^{*}{ }^{*}{ }^{2}(\mathrm{R} 296)\end{array}$ \\
\hline $96 c$. & & $\frac{\text { kaigu }}{360)}$ (GS & & $\begin{array}{l}\text { kai }= \\
\text { qai }^{\text {Tas }} / \mathrm{E}\end{array}$ & & & $\begin{array}{l}<\operatorname{Tn}^{*} x a i \\
(\mathrm{AX} 156) \\
\end{array}$ \\
\hline 96d. & & & & & $d \partial_{\mathrm{D}}$ & & $\begin{array}{l}\text { cf. } d \underline{\underline{\partial}}, d^{\circ} \text { der, } \\
\text { er? }\end{array}$ \\
\hline $\begin{array}{l}\text { 97a. } \\
\text { white }\end{array}$ & sear & siroi $i^{\mathrm{Ch}}$ & sera'a & $\begin{array}{l}\operatorname{ser}^{\text {Tas }}= \\
\text { seri }^{\text {Tas }} / \text { E }\end{array}$ & $\begin{array}{l}\text { siri }= \\
\text { säri, sirk }{ }_{\mathrm{D}}\end{array}$ & & $\begin{array}{l}{ }^{*} \text { ser }(-):{ }^{*} \text { ser ice } \\
(\mathrm{J} 138 ; \mathrm{H} \text { 874) } \\
<\mathrm{U} \text { *'śar3 } \\
(\mathrm{R} 464)\end{array}$ \\
\hline $97 \mathrm{~b}$. & & $\begin{array}{l}\text { jogudad- } \\
d e^{\mathrm{B}}\end{array}$ & dekagá ${ }_{\text {Pallas }}$ & $\begin{array}{l}\text { čag } \\
t a ̈ g \mathrm{MO} \\
\check{c}{ }^{\mathrm{C}} k^{\mathrm{Tas}}\end{array}$ & & & ${ }^{*} j e k 3$ (J 42) \\
\hline $97 \mathrm{c}$. & & & & & $d^{\prime} / t^{\prime} a \bar{r} \partial \hat{\partial} k_{\mathrm{D}}$ & & $\begin{array}{l}<\text { Tat, Alt } \\
\text { jaryq }\end{array}$ \\
\hline 97d. & & & & & $\underline{\bar{o} i}{ }_{\mathrm{D}}$ & & $\begin{array}{l}<\text { Tat } o i \\
\text { weissblau } \\
\text { (Pferdefarbe) } \\
\text { (Jo 243) }\end{array}$ \\
\hline $97 \mathrm{e}$. & & & & & & $\underline{k i r}^{\mathrm{Mt}}$ & $\begin{array}{l}<\mathrm{Tk}^{*} q y r \\
\text { grey (H 494) }\end{array}$ \\
\hline $\begin{array}{l}\text { 98a. } \\
\text { who }\end{array}$ & śa & $\begin{array}{l}\text { sio, } \text { sie }^{\mathrm{Ch}} \\
\text { sea }^{\mathrm{B}} / \mathrm{Te}\end{array}$ & sele & & & & $\begin{array}{l}{ }^{*} k i-/^{*} k i-(\mathrm{J} 69) \\
<\mathrm{U}{ }^{*} k e{ }^{*} k i \\
(\mathrm{R} 140)\end{array}$ \\
\hline 98aa. & 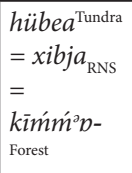 & & & & $\begin{array}{l}\text { šimdi }= \\
\text { šind }_{\mathrm{D}}^{\circ}\end{array}$ & kym $^{\mathrm{Mt}}$ & $\begin{array}{l}{ }^{*} k i-{ }^{*} k i-m \ddot{a} \\
(\mathrm{~J} 69 ; \mathrm{H} 486) \\
<\mathrm{U}{ }^{*} k e{ }^{*} k i \\
(\mathrm{R} 140)\end{array}$ \\
\hline 98ab. & $k u^{\prime} k k_{L_{\mathrm{Le}}}$ & $\begin{array}{l}\text { (hôke } \\
\text { which } \\
\left.=k o k e_{\mathrm{So}}\right)\end{array}$ & $\begin{array}{l}\text { (kua } \\
\text { which) }\end{array}$ & kut, kud & $\begin{array}{l}\text { ( } g / k i d i \\
\text { which ; } \\
\text { cf. gi' } i \text { D } \\
\text { which of } \\
\text { two) }\end{array}$ & $\begin{array}{l}\left(k u \lg u^{\mathrm{Mt}}\right. \\
\text { which })\end{array}$ & $\begin{array}{l}{ }^{\star} k u-(\mathrm{J} 75 ; \\
\mathrm{H} 563,576) \\
<\mathrm{U}{ }^{*} k u-{ }^{*}{ }^{*} k o- \\
(\mathrm{R} 191)\end{array}$ \\
\hline
\end{tabular}


On the classification of the Samoyedic languages

\begin{tabular}{|c|c|c|c|c|c|c|c|}
\hline gloss & Nenets & Enets & Nganasan & Selkup & Kamas & Mator & etymology \\
\hline \multirow[t]{2}{*}{$\begin{array}{l}\text { 99a. } \\
\text { wo- } \\
\text { man }\end{array}$} & $n e_{\mathrm{RNS}}$ & $\begin{array}{l}n \hat{e}^{\mathrm{Ch}} \\
n e^{\mathrm{B}} / \mathrm{Pr} \\
n \bar{e}^{\mathrm{K}} / \mathrm{Pu}\end{array}$ & $n \hat{e}=n \bar{l}_{\mathrm{M}}$ & $\begin{array}{l}n e \ddot{a}^{\mathrm{OO}, \check{\mathrm{C}}}= \\
n e^{\mathrm{Tas}} / \mathrm{E}\end{array}$ & $n \mathcal{E}, n \bar{\varepsilon}, n \bar{e}_{\mathrm{D}}$ & $\begin{array}{l}n e^{\mathrm{Mt}} \\
\text { Weibs } \\
\text { Person } \\
\left.n e^{\mathrm{Kg}} \text { uxor }\right)\end{array}$ & $\begin{array}{l}{ }^{*} \text { ne (J 100; } \\
\mathrm{H} 727)<\mathrm{U} \\
{ }^{*} \text { ninä (R 305- } \\
06) \neq \mathrm{U}{ }^{*} \text { näxi } \\
\left(\mathrm{S}_{1} 539\right)\end{array}$ \\
\hline & & & & $\begin{array}{l}e m a^{\mathrm{MO}} \\
i m a^{\mathrm{B}, \mathrm{Tas}}= \\
i m a^{\mathrm{Tas}} / \mathrm{E}\end{array}$ & & $\begin{array}{l}\left(e m e^{\mathrm{Tg}}\right. \\
\text { imang }^{\mathrm{Mt}} \\
\text { mother })\end{array}$ & $\begin{array}{l}\text { *emä mother } \\
(\mathrm{J} 23 ; \mathrm{H} 64)< \\
\mathrm{U}{ }^{*} \text { emä } \\
\left(\mathrm{R} 74 ; \mathrm{S}_{1} 536\right)\end{array}$ \\
\hline $\begin{array}{l}\text { 100a. } \\
\text { yellow }\end{array}$ & $\begin{array}{l}\text { tâsihaei }= \\
\text { tasej }_{\mathrm{RNS}}\end{array}$ & $\begin{array}{l}\text { taserad- } \\
d e^{\mathrm{Ch}}\end{array}$ & \begin{tabular}{|l|} 
tod’akuo $_{\mathrm{NRN}}$ \\
tos rüu- \\
tüo \\
gelblich) \\
gellich
\end{tabular} & & & & \\
\hline $100 \mathrm{~b}$. & & $\begin{array}{l}\text { takuda- } \\
d e^{\mathrm{Ch}} / \mathrm{x}\end{array}$ & & & & & (X 130) \\
\hline $100 \mathrm{c}$. & & & & patill $^{\text {las }}{ }_{\mathrm{E}}$ & & & $\begin{array}{l}\text { cf. pati bile, } \\
\text { gall < } \\
{ }^{\star} \text { pâtä (J 115) }\end{array}$ \\
\hline 100d. & & & & & $\underline{s e g}_{\mathrm{D}}$ & & $\begin{array}{l}\text { Soy, Sag sigän } \\
\text { grass }\end{array}$ \\
\hline $100 \mathrm{e}$. & & & & & & $\begin{array}{l}\text { táinaha }^{\mathrm{Mt}} \\
\text { tánjaha }^{\mathrm{Kg}}\end{array}$ & (H 945) \\
\hline $100 f$. & & & & & $\begin{array}{l}\underline{d e} z i_{\mathrm{D}} \\
\text { (+ of } \\
\text { copper) }\end{array}$ & & \begin{tabular}{|l} 
cf. des cop- \\
per, bronze \\
(Jo 121)
\end{tabular} \\
\hline $100 \mathrm{~g}$. & & & & & $\begin{array}{l}\frac{s \bar{a} r}{\mathrm{D}} \\
(+ \text { grau- } \\
\text { lich })\end{array}$ & $\underline{\operatorname{sary} x}$ & \begin{tabular}{|l}
$<$ Tk: Khak \\
saryy yellow, \\
Tat sāryg \\
(H 851)
\end{tabular} \\
\hline
\end{tabular}

Note 1: The main source of Nenets, Enets, Nganasan, Selkup and Kamas is Castrén 1855; for Mator it is Helimski 1997 (= H). The additional sources are: Nenets - Le, NRS, RNS; Enets - M, X; Nganasan - KS, NRN, Xelimskij 1994; Selkup - DA, E, G; Kamas - D.

Note 2: Forms in parentheses differ in semantics and for this reason are not calculated as cognates. The underlined forms are loanwords.

Abbreviations of languages: Alt Altai, Brb Baraba, Bsk Bashkir, Chag Chaghatai, Ewk Ewenki, F Finnic, Fi Finnish, FU Finno-Ugric, Hu Hungarian, Kalm Kalmyk, Kb Koibal, Kg Karagas, Khak Khakas, Km Kamas, Kzk Kazakh, Ma Mansi, Md Mordvin, Mo Mongolian, Mt Mator, N North, Ru Russian, Sag Sagai, Sm Samoyedic, Soy Soyot, Tat Tatar, Tg Taigi, Tk Turkic, Tn Tungusic, U Uralic, Yak Yakut, Yen Yeniseic, Ykg Yukaghir. 


\section{Václav Blažek}

Abbreviations of dialects (written in the upper index):

Nenets: Knd Kondin, Obd Obdorsk.

Enets: B Baicha, Ch Khantaika, K Karasino.

Selkup: B Baicha, Č Čaja, K Ket', Kar Karasin, MK Middle Ket', MO Middle Ob, N Narym, NP Nats Pumpokolsk, OO Upper Ob, Tu Tundra, UK Upper Ket'.

Abbreviations of authors (written in the lower index):

Gl Gluxij, La Labanauskas, Le Lehtisalo, Pop Popov, Pr Prokofjev, Pu Pusztay, So Sorokina, Su Susekov, Te Tereščenko, VS Volodin + Sorokina, X Xelimskij. These authors are usually cited according to Janhunen 1977 (J), Mikola 1995 (M) or Xelimskij 1982 (X).

\section{Conclusion}

The most important result of the present contribution is a dating of the disintegration of Samoyedic to the 3rd-2nd century BCE, i.e. between the disintegrations of its neighbours, Ob-Ugric around 400 BCE and Turkic around 100 BCE. Applying the cladistic strategy based on average values of percentages expressing mutual relations, two primarily parallel branches of the Samoyedic dialect continuum may be determined: North Samoyedic vs. South Samoyedic, consisting of Selkup and Sayan Samoyedic. With regard to the thin interval of $1.4 \%$ between the disintegration of Common Samoyedic and separation of Selkup from Kamas and Mator, it is possible to consider the alternative trichotomic model: North Samoyedic, Selkup and Sayan Samoyedic. Relatively low scores of Nganasan with all other languages (with exception of Enets) probably indicate an influence of a pre-Samoyedic substratum of the Taimyr Peninsula. On the other hand, the possibility that the Sayan Samoyedic branch was secondarily "formed" thanks to the geographical neighbourhood of Kamas (and Koibal) and Mator (and Taigi and Karagas) is not excluded. In this case, Helimski's $(1982=\mathrm{X})$ idea of four coordinate branches would be confirmed.

Concerning the Samoyedic-Finno-Ugric relations, their disintegration dated to 3400 BCE is in agreement with communis opinio (see the discussion in Blažek 2012: 30-32). The most remarkable result is that the order of lexical proximity of individual Finno-Ugric branches to Samoyedic does not correspond with the geographical distance - on the basis of the core lexicon, the closest relative of Samoyedic is Finnic (every Finnic language shares with Samoyedic, on average, a higher score than all other branches): 
1. Finnic: $28.62 \%$

2. Mari: $26.12 \%$

3. Permic: $25.95 \%$

4. Ugric: $24.03 \%$

5. Saamic: $22.95 \%$

6. Mordvinian: $22.39 \%$

This result does not confirm the idea of an Eastern Uralic superbranch consisting of Ugric and Samoyedic (Häkkinen 2007, 2009; Parpola 2012: 288), at least in a genealogical sense. On the other hand, an areal explanation operating with a West Siberian Sprachbund consisting of Ugric, Samoyedic and probably also the Yeniseic language Pumpokol, all characterized by the isogloss ${ }^{*} s{ }^{*} t$, was already formulated by Helimski in 1982 (X 124). The specific Finnic-Samoyedic relations represent an open question. As working hypotheses, the following scenarios may be offered: (i) Primary neighbourhood in the Uralic dialect continuum. (ii) Independently shared archaisms in the northwest and northeast peripheries of the Uralic dialect continuum. (iii) Substratum common to both Samoyedic and Finnic. (iv) Finnic-like substratum in Samoyedic (with regard to high scores of the Nganasan core lexicon with all Finno-Ugric branches, the hypothetical pre-Samoyedic substratum in Nganasan could be of Uralic origin too). (v) Samoyedic-like substratum in Finnic (and not Saamic, where it did not become evident in the core lexicon). Only future research can solve this challenge.

Václav Blažek Department of Linguistics and Baltic Studies Faculty of Arts, Masaryk University, Brno Czech Republic

\section{Acknowledgement}

The present study was prepared thanks to a grant from the The Czech Science Foundation (GAČR), GA15-12215S. The author is grateful to two anonymous referees for their valuable corrections and recommendations and to John D. Bengtson for his correction of English. 


\section{Václav Blažek}

\section{Notes}

1. Already Janhunen $(1983,119)$ identified in Samoyedic *sejptâ ' 7 ' a loan from Common Tocharian ${ }^{\star}$ șptá id. (Ringe 1996: 67). It is possible to add North Samoyedic *menüjâ 'full moon' (Xelimskij 1978: 126; 1984: 117; 2000: 400) < Common Tocharian *myeñ - 'moon' (Ringe 1996: 108-109), and Sayan Samoyedic *pey- 'to write, impress' (XE 119; H 329) < Tocharian AB (present stem) pink- 'to write, paint, delineate' (Adams 2013: 410); cf. also Napol'skikh 2001: 373, 371.

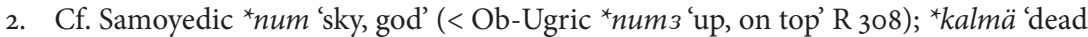
body, grave' (continuants of $\mathrm{U}^{\star}{ }^{\star} k a l m a$ have been lost in the Ugric branch - see $\mathrm{R}$ 119-120; Janhunen 1998: 477 assumes their existence).

3. Janhunen (1998: 477) quotes Samoyedic *juntâ 'horse' and ‘jür '10o' as examples of proto-Bolgar loanwords. It is possible to add another numeral, Samoyedic ${ }^{\star} t e(j) t t \hat{~}$ '4' (J 159), resembling Volga-Bolgar *tüät, Chuvash tăvattă, derived from protoTurkic protoform ${ }^{*} d \bar{r} r t$ ' 4 ' (Blažek 1996-97: 6-7; 1997: 37; 1999: 93,105). But in the proto-Samoyedic language there is no witness to the cluster ${ }^{*}-r t-(\mathrm{cf}$. S 46-47). This

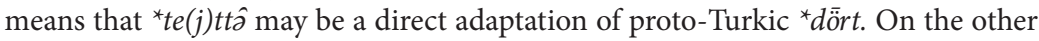
hand, $-r$ - is also missing outside the Bolgar-Chuvash branch, e.g. in Uighur dial. tööt, Lobnor dö(j)t/töjt, Salar $\delta \ddot{o t}$, $\delta o t$ id. (Sevortjan 1980: 284-86).

\section{References}

$\mathrm{A}_{1}=$ AIKIO, ANTE 2002: New and Old Samoyed Etymologies. - Finnisch-Ugrische Forschungen 57. 9-57.

$\mathrm{A}_{2}=$ AIKIO, ANTE 2006: New and Old Samoyed Etymologies. - Finnisch-Ugrische Forschungen 59. 9-34.

Adams, Douglas Q 2013: A Dictionary of Tocharian B. Revised and Greatly Enlarged, Vol. I-II. Rodopi. Amsterdam - New York.

BLAžEK, VÁClAV 1996-97: Some thoughts about Uralic Numerals. - Philologia FennoUgrica 2-3. 1-18.

- 1997: Altaic Numerals. - Studia Etymologica Craciviensia 2. 33-75.

- 1999: Numerals. Comparative-etymological analyses and their implications. Masaryk University. Brno.

- 2012: Was there a Volgaic unity within Finno-Ugric? - Finnisch-Ugrische Forschungen 61. 29-91.

BLAŽEK, VÁClAV \& Kovář, MiChal 2013: Z historie uralistiky. Pionýrské období (9. st.1850). - Linguistica Brunensia 61. 273-292.

BlAŽEK, VÁClAV \& SCHWARZ, Michal 2011: Tocharians. Who were, where they came from and where they lived. - Michal Schwarz (ed.): Tocharian Studies. Masarykova univerzita. Brno. 113-147.

Blažek, Václav, Hofírková, Lucie \& Kovář, Michal 2011: Rané prameny o národech uralské jazykové rodiny. - Linguistica Brunensia 59. 189-224.

Castrén, Matthias Alexander 1855: M. Alexander Castrén's Wörterverzeichnisse aus den samojedischen Sprachen. Herausgegeben von Anton Schiefner. Eggers \& Comp. St. Petersburg. 


\section{On the classification of the Samoyedic languages}

Collinder, BJöRn. 1960. Comparative Grammar of the Uralic Languages. Almqvist \& Wiksell. Stockholm.

D = Donner, KAI 1944: Kamassisches Wörterbuch, bearbeitet und herausgegeben von Aulis J. Joki. Lexica Societatis Fenno-Ugricae VIII. Helsinki.

DA = Donner, Kai \& Alatalo, Jarmo (ed.) 2004: Sölkupisches Wörterbuch aus Aufzeichnungen von Kai Donner, U. T. Sirelius und Jarmo Alatalo. Lexica Societatis Fenno-Ugricae XXX. Helsinki.

Dүво 2006 = Дыбо, Анна В.: Хронология тюркских языков и лингвистические контакты ранних тюрков. - Сравнительно-историческая грамматика тюркских языков. Пратюркский язык-основа. Картина мира пратюрков. Отв. ред. Э. Р. Тенишев \& А. В. Дыбо. Наука. Москва. 766-817.

E = ERdÉLYI, IsTván 1969: Selkupisches Wörterverzeichnis. Akadémiai Kiadó. Budapest. Ethnologue: Languages of the World, Seventeenth edition. Ed. by Lewis, M. Paul, Gary F. Simons, and Charles D. Fennig. SIL International. Dallas, Texas, 2013. Online version: <http://www.ethnologue.com>.

$\mathrm{G}=$ Григоровский, НиколАй П. 2007: Южноселькупский словарь Н. П. Григоровского. Ed. by E. Helimski. Hamburger Sibirische und Finnougrische Materialien, Bd. 4. Hamburg.

GS = Castrén, Matthias Alexander 1854: Grammatik der Samojedischer Sprachen. Hrsg. von Anton Schiefner. Eggers (Kaiserliche Akademie der Wissenschaften). St. Petersburg. <http://books.google.cz/books?id=AbwKAAAAIAAJ\&redir_esc=y> H = Helimski, Eugene. 1997. Die matorische Sprache. Szeged: Studia uralo-altaica 41.

HÄKKINEN, JААKко 2007: Kantauralin murteutuminen vokaalivastaavuuksien valossa.

Helsingin yliopisto: Suomalais-ugrilaisen kielentutkimuksen pro gradu -työ.

$<$ http://urn.fi/URN:NBN:fi-fe20071746>

- 2009: Kantauralin ajoitus ja paikannus - perustelut puntarissa. Journal de la Société Finno-ougrienne 92. 9-56.

J = JANHUnEN, Juha 1977: Samojedischer Wortschatz. Castrenianumin toimitteita 17. Helsinki.

Janhunen, Juha 1998: Samoyedic. - ULA = The Uralic Languages, ed. by Abondolo, DANIEL. Routledge. London. 1998. 457-479.

- 2009: Proto-Uralic - What, where, and when? - Quasquicentennial of the FinnoUgrian Society. Mémoires de la Société Finno-Ougrienne 258. Helsinki. 57-78.

JJ = Janhunen, Juha 1982: On the Structure of Proto-Uralic. - Finnisch-Ugrische Forschungen 44. 23-42.

JN = JANHUNEN, JUHA 1976: Adalékok az északi-szamojéd hangtörténethez: vokalizmus az első szótagi magánhangzók. - Néprajz és Nyelvtudományi Társaság Kiadványai 19-20. 165-188.

Jo = JoKI, Aulis 1952: Lehnwörter der Sajansamojedischen. Mémoires de la Société Finno-Ougrienne 103. Helsinki.

KS = KoRTt, I. R., SimčEnko, Ju. B. 1985: Wörterverzeichnis der nganasanischen Sprache. Teil 1. Systemata mundi. Berlin.

$\mathrm{KU}=$ XELIMSKIJ 2000.

Le = LeHTISALO, T. 1956: Juraksamojedisches Wörterbuch. Lexica Societatis Fenno-Ugricae XIII. Helsinki. 


\section{Václav Blažek}

M = MiкоLa, Tiвor 1995: Morphologisches Wörterbuch des Enzischen. Studia uraloaltaica 36. Szeged.

Mallory, James P. \& Mair, victor H. 2000: The Tarim Mummies. Ancient China and the Mystery of the Earliest Peoples from the West. Thames \& Hudson. London.

MUdRAK 2009 = МудРАк, Олег А.: Классификация тюркских языкков и диалектов с помощю методов глоттохронологии на основе вопросов по морфологии и исторической фонетике. Москва: Российский государственный гуманитарный университет.

NAPOLSKIKH, Vladimir 2001: Tocharisch-uralische Berührungen: Sprache und Archäeologie. - Early Contacts between Uralic and Indo-European: Linguistic and Archaeological Considerations, ed. by C. Carpelan, A. Parpola, P. Koskikallio. Mémoires de la Société Finno-Ougrienne 242. Helsinki. 367-383.

$\mathrm{NRN}=$ Костеркина, НАдежда Т., Момде, Александр Ч. \& Жданова, ТАтьяна, Ю. 2001: Нганасанско-русский и русско-нганасанский словарь. Санкт-Петербург. $<$ http://wwwuser.gwdg.de/ mkatzsc/Dt-Ng-Woerterverzeichnis.pdf>

NRS = Ненецкко-русский словарь, ed. Н. М. ТЕРЕщЕнко. Советская энциклопедия. Москва 1965.

PARPOLA, AsKo 2012: The problem of Samoyed origins in the light of archaeology: On the formation and dispersal of East Uralic (Proto-Ugro-Samoyed). - Per Urales ad Orientem. Iter polyphonicum multilingue. Festskrift tillägnad Juha Janhunen på hans sextioårsdag den 12 februari 2012, edited by Tiina Hyytiäinen, Lotta Jalava, Janne Saarikivi \& Erika Sandman. Mémoires de la Société Finno-Ougrienne 264. Helsinki. 287-298.

$\mathrm{R}=$ Uralisches etymologisches Wörterbuch, ed. KÁroly RÉDEI. Akadémiai Kiadó. Budapest 1988.

Rä = RäsÄNEN, MARTTI 1969: Versuch eines etymologischen Wörterbuchs der Türksprachen. Lexica Societatis Fenno-Ugricae XVII, 1. Helsinki.

Ringe, Don 1996: On the chronology of sound changes in Tocharian, Vol. 1: From Proto-Indo-European to Proto-Tocharian. American Oriental Series, Vol. 80. American Oriental Society. New Haven

RNS = Русско-ненецикий словарь, ред. А. П. ПыРеркА \& Н. М. ТерещЕнко. Государственное издательство иностранных и национальных словарей. Москва 1948.

Ruhlen, Merritt 1987: A Guide to the World's Languages, Vol. 1: Classification. Stanford University Press. Stanford.

S = Sammallahti, Pekka 1979: Über die Laut und Morphemstruktur der uralischen Grundsprachen. - Finnisch-Ugrische Forschungen 43. 22-66.

$\mathrm{S}_{1}=$ Sammallahti, PekKa 1988: Historical Phonology of the Uralic Languages. - ULS = The Uralic Languages. Description, history and foreign influences, ed. by DeNIs SinOR. Brill. Leiden - New York - København - Köln 1988. 478-554.

Schwarz, Michal \& BlažEK, VÁClaV 2011: Klasifikace a přehled turkických jazyků. Linguistica Brunensia 59, 25-59.

SEVORTJAN 1980 = Севортян, Геворк: Этимологический словарь тюркских языков, III. Наука. Москва.

$\mathrm{St}=$ СтАРОстин, Сергей A 1982: Праенисейская реконструкция и внешние связи енисейских языков. - Кетский сборник, ed. Е. А. Алексеенко. Наука. Ленинград. 144-237. 


\section{On the classification of the Samoyedic languages}

Starostin, Sergei 2004: Preliminary results of application of 'recalibrated' glottochronology to classification of Eurasian language families. Paper presented at Workshop on Prehistoric Chronology: Language, Genes and Migrations being held at the Santa Fe Institute, March 2004.

STIPA, GÜNTER J. 1981: Vom ostseefinnischen bis zum Samojedischen reichender Lehnwortschatz im „Russian Vocabulary“ (1618-19) des Richard James. - Journal de la Société Finno-ougrienne 77, 4. 41-66.

Svyatko, Svetlana, James P. Mallory, Eileen M. Murphy, Andrey V. Polyakov, Paula J. Reimer \& Rick J. Schulting 2009: New radiocarbon dates and a review of the chronology of prehistoric populations from the Minusinsk Basin, Southern Siberia, Russia. - Radiocarbon 51/1. 243-273.

TMS Сравнительный словарь тунгусо-маньчжурских языков, I-II, ред. Вера I. Цинциюс. Наука. Ленинград 1975-1977.

VADECKAJA 1990 = ВАдецкая, Э. Б.: Южно-самодийские компоненты культуры древнего населения Присаянья. - Uralo-Indogermanica II, ред. В. В. Иванов и др. Институт славяноведения и балканистики. Москва. 71-8о.

$\mathrm{X}=$ Хелимский, Евгений А. 1982: Древнейшие венгерско-самодийские языковые параллели: Лингвистическая и этногенетическая интерпретация. Наука. Москва.

$\mathrm{XE}=$ Хелимский, Евгений А. 1992-93: Etymologica 49-79: Материалы по этимологии маторско-тайгийско-карагасского языка. - Nyelvtudományi közlemények 93, 101-123. <http://www.uni-hamburg.de/onTEAM/grafik/1264671657/Publikationsliste.pdf $>$

XeLIMSKIJ 1978 = Хелимский, Евгений А.: Реконструкция прасеверносамодийских (ПСС) лабиализированых гласных непервых слогов. - Проблема реконструкиии. Конференция (тезисы докладов). Наука. Москва. 123-126.

- 1982 = Хелимский, Евгений A.: Keto-Uralica. - Kетский сборник, ed. Е.А. Алексеенко. Наука. Ленинград. 238-250.

- 1984 = Хелимский, Евгений А.: Две заметки о славянско-самодийских аналогиях. - Балто-славянские исследования 1983. 114-123.

- $[2000]=$ Хелимский, Евгений А.: Самодийская лингвистическая реконструкция и праистория самодийцев. - KU 13-25.

- 1994 = Хелимский, Евгений А.: Указатель слов к текстам [нганасанских] камланий. - Таймырский этнолингвистический сборник, ed. Евгений Хелимский. Российский государственный гуманитарный университет. Москва. 107-146.

- 2000 = Хелимский, Евгений А.: Компаративистика, уралистика: Лекции и статьи. Языки русской культуры. Москва. 